\title{
Traditional uses, phytochemistry and pharmacology of genus Fritillaria-a review
}

Ishrat Rashid ${ }^{1}$ and Ubaid Yaqoob ${ }^{2 *}$

\begin{abstract}
Background: Genus Fritillaria is one among the biggest genera of family Liliaceae comprising of around 130-165 species. Fritillaria is viewed as a significant genus and a source of significant pharmaceutically active compounds utilized in conventional drugs by folklore. Fritillaria is utilized worldwide as medication and food. Different chemically dynamic components separated from genus Fritillaria, their phytochemistry with structure and pharmacology of these compounds have been extensively reviewed.

Main body: Fritillaria is utilized for treatment of dyspepsia, chest injury, tuberculosis, cough, asthma, gout, bronchitis, dysuria, sinus, boils, stomatitis, malaria, insanity, anaemia, immunity promoter, remedy for child emaciation, fever, burning sensation, phthisis and broncho-asthma, heart diseases, dysfunction of breathing and nervous system, etc. Different chemical components isolated from genus Fritillaria include around 120 alkaloids, 15 terpenoids as well as saponins, glycosides, volatile components, nucleosides, amino acids, nucleobases, flavonoids, fatty acids and so forth.

Conclusions: Many Fritillaria species have been utilized in traditional Chinese medication on account of their effects of clearing heat, moistening the lung, alleviating cough, asthma, tumours, scrofula and so on. Fritillaria is utilized for treatment of dyspepsia, chest injury, tuberculosis, cough, asthma, gout, bronchitis, dysuria, sinus, boils, stomatitis, malaria, insanity, anaemia, immunity promoter, remedy for child emaciation, also for fever, burning sensation, phthisis and broncho-asthma, heart diseases, dysfunction of breathing and nervous system, etc.
\end{abstract}

Keywords: Antitussive, Chemical compounds, Expectorant, Fritillaria, Pharmacology, Phytochemistry

\section{Background}

Genus Fritillaria L. is one among the largest genera belonging to monocot family Liliaceae comprising of around 130-165 species (Rix 2001; Xiao et al. 2007), native to mild zone of the Northern Hemisphere (Tsukamoto et al. 1989; Hao et al. 2013). The centre of genetics diversity of the genus has been reported to lie in Iran, where subgenra from the central Asia, Mediterranean and Caucasus meet (Rix 1977). Some species are native to Cyprus, Iran and southern Turkey (Ori et al. 1992a), about 18 species are reported endemic to Iran (Khaniki 2003) and about 20 species had been reported in China,

\footnotetext{
*Correspondence: ubaidyaqoob@spcollege.edu.in

2 Department of Botany, Sri Pratap College, M. A. Road, Srinagar, J\&K

190001, India

Full list of author information is available at the end of the article
}

till 1980 (Chen 1980). Fritillaria is regarded as an important genus in Liliaceae family and a plant source of significant chemically components utilized in conventional prescriptions by folklore of Turkey (Farooq et al. 1994), South East Asia (Zhou et al. 2010) China, Pakistan and Japan (Kaneko et al. 1981b). Fritillaria species are presently popular in therapeutic plants industry (Day et al. 2014) and floriculture (Turktas et al. 2012). Fritillaria is utilized worldwide as medication and food; typically roasted bulbs of certain species are utilized as food by Native Americans.

Bulbus Fritillaria usually called as " Pei-mu " or "Bei$\mathrm{mu}$ " in Chinese language and in Japanese as "Bai-mo" (Chi et al. 1936; Kitajima et al. 1982a), obtained from the bulbs of different species of the genus Fritillaria (Liliaceae), has been utilized as an expectorant and antitussive in customary Chinese medication for over 2000 years 
(Kaneko et al. 1988; Shang and Liu 1995). Officially, natural Beimu is prepared by utilizing the bulbs of nine distinctive Fritillaria species in particular Fritillaria unibracteata Hiao et Hsia, Fritillaria thunbergii Miq., Fritillaria cirrhosa D. Don, Fritillaria delavayi Franch, Fritillaria przewalskii Maxim ex Batal, Fritillaria ussuriensis Maxim., Fritillaria pallidiflora Schrenk, Fritillaria walujewii, and Fritillaria hupehensis Hsiao et K. C. Hsia. In Chinese folk medication, other Fritillaria species were additionally utilized as the sources for Beimu in local regions of China (Shang and Liu 1995).

\section{Main text}

\section{Traditional uses}

Bulbus Fritillaria have been utilized as main Chinese crude drugs and furthermore as an antihypertensive and antiasthmatic drugs from years. Notwithstanding, Fritillaria species vary in their phytochemicals with various pharmacological impacts (Hao et al. 2013). In conventional medication, many species of Fritillaria have been utilized by Japanese (Ito et al. 1963; Kaneko et al. 1981b), Pakistani, Turkish (Farooq et al. 1994) and south-east Asian individuals as herbal remedies (Qian and Nohara 1995; Akhtar et al. 2003; Zhou et al. 2010). Fritillaria are utilized worldwide as medication and food; normally roasted bulbs of some species are utilized as food by Native Americans (Orhan et al. 2009). Prior it was exported from Nepal to India and China due to its high therapeutic values (Thomson 2007). Genus Fritillaria have been utilized for long due to their effects of moistening the lung, clearing heat, resolving phlegm, soothing cough, remedy for cough brought about by lung heat and dryness, a cough because of a yin weakness, sputum with blood and a low sputum dry cough. The bulb part of Fritillaria species utilized as decoction or in dried form to cure bronchitis, cough, tumours, struma, asthma, haemoptysis and insufficiency of milk (Perry 1980; Kang et al. 2002). Fritillaria have been utilized to cure numerous lung infections, including tuberculosis, and asthma. Moreover, it is used as a lymphatic decongestant to decrease glandular or nodular breast tissue, goitre, swellings and lymphadenopathy. It has been utilized for the treatment of prolonged hypotension, sensory system, defective breathing and incitement of the heart muscle (Erika and Rebecca 2005a) as well as treating swelling underneath the skin, for example, scrofulous swellings and breast nodules (Da-Cheng et al. 2013). It is likewise detailed that blood platelet conglomeration is restrained by Fritillaria bulbs.

Fritillaria ebeiensis G. D. Yu and G. Q. Ji, local to northwest area of Hubei region, China is utilized as medication for saturating lungs, clearing heat and throat infections, for example cough, tracheitis and asthma, by folklore of
China (Li et al. 1994). Fritillaria ebeiensis shows high antitussive and expectorant impacts (Yu et al. 1985). A crude drug known as Ebeibeimu is set up by treating the bulbs of F. ebeiensis with lime and, afterward bleached in sun, serves as a substitute for major conventional Chinese medication Beimu (Wu et al. 1995). Fritillaria cirrhosa, Fritillaria thunbergii and Fritillaria pallidiflora are accounted for to be utilized in various cough status with respect to their potencies in customary Chinese medication, great quality bulbs of well-grown $F$. cirrhosa, when dried seem white and fine (Bensky and Gamble 1993; Konchar et al. 2011) and bulbs can be utilized as entire or in powdered form as remedies for clearing the lungs from mucus and cooling heat (Bensky et al. 2004; $\mathrm{Li}$ et al. 2006a, 2009). It has been utilized to cure diseases like asthma and cough in TCM (Traditional Chinese Medicine) for over 2000 years (Wang et al. 2011) and furthermore act expectorant, astringent and demulcent (Uprety et al. 2010). Pharmaceutical investigations of Fritillaria thunbergii Miq. have revealed that it has been utilized to deal with different infections like cough, disposing of mucus, alleviating pain and anti-inflammatory problems (Qian and Xu 1985; Xiao et al. 1992; Zhou et al. 2003). Fritillaria thunbergii Miq. (known as 'Zhe Beimu' in Chinese) is among the main species from genus Fritillaria to be utilized in TCM (Traditional Chinese Medicine) as expectorant and antitussive herb for over 200 years (Li et al. 2006a). F. pallidiflora is an ordinarily utilized plant for cough treatment in TCM. Fritillaria pallidiflora Schrenk generally found in Xinjiang region of China is utilized as an antitussive, expectorant and antiasthmatic medication (Xu et al. 1990a; Li et al. 1993; Zhou et al. 2003). Bulbus Fritillariae ussuriensis (BFU) in view of its antiasthmatic, expectorant and antitussive actions is utilized as food and orthodox medication, scattered all through the Northeast areas of China, including Liaoning, Heilongjiang and Jiling areas and also for treating swollen throat and lung diseases in Chinese medication (Perry 1980). F. maximowiczii (Rinyou-Baimo), local to north-eastern part of China, is referred to act as an alternative for the bulb of various Fritillaria species like Fritillaria thunbergii (Setu-Baimo), Fritillaria unibracteata, Fritillaria taipaiensis (Sen-Biamo) and Fritillaria cirrhosa used to treat cough. In customary traditions, Fritillaria imperialis has been utilized for the treatment of different diseases like asthma, pharyngitis, bronchitis, cough, struma, haemoptysis, dysuria and gland tumour (Bailey 1966; Perry 1980). Its tendrilled bulbs are utilized as a home remedy for haemorrhage, cough and mucus, treatment of abscess, high fever, absence of milk, eye sickness, and rheumatoid arthritis (Aydın et al. 2018) and act as antianxiety/depression (Abbaszadeh et al. 2019). Fritillaria roylei is utilized to prepare an ayurveda 
drug Ashtavarga (Ashta-eight and varga-group); thus, Ashtavarga is a polyherbal formulation and $F$. roylei is likewise utilized for the preparation of ashtavarga with name Kakoli (Warrier et al. 1994; Singh 2006; Negi et al. 2007). It additionally shows impacts of cooling and spermopiotic, antirheumatic, antiasthmatic, galactagogue, haemostatic, antipyretic and oxytocic properties (Singh 2006). Its rhizome acts as expectorant, sexual stimulation, spermatogenic and tonic. Restoratively it is utilized for the treatment of dyspepsia, chest injury, tuberculosis, cough, asthma, congenital pulmonary haemorrhage, gout, bronchitis, diarrhoea, dysuria, sinus, boils, stomatitis, malaria, insanity, anaemia, oligospermia (low sperm check), immunity promoter, remedy for child emaciation, antidote for spider poisoning (Balkrishna 2012) and furthermore for fever, burning sensation, phthisis (Singh 2006) and broncho-asthma (shaheen et al. 2014). It is additionally utilized for the treatment of incitement of the heart muscle, heart diseases, decreased pulse rate, defective breathing and nervous system (Erika and Rebecca 2005b). Fritillaria hupehensis Hsiao et K.C. Hsia, named 'Hubeibeimu', documented in the pharmacopeia of the people Republic of China, is commonly utilized in orthodox medication and very much found in Northwest region of Hubie, China. Bulbs of Fritillaria unibracteata 'Chuan Bei-Mu', utilized as cough reliever, antiasthmatic and decongestant agents for long time in conventional Chinese medication also included in the Chinese Pharmacopeia (Liang 2004), are utilized to treat asthma (Shou et al. 2009). One of the sources for BFC (Bulbus F. cirrhosa) is bulbs of Fritillaria wabuensis S. Y. Tang and S. C. Yueh (BFW), which is taken orally to cure cough by customary individuals. It has likewise been broadly utilized in China to cure asthma and cough in clinic because of its positive therapeutic impacts and lesser side effects (Wang et al. 2012). Fritillaria tortifolia X. Z. Duan et X. J. Zheng native plant in Xinjiang Uygur area is utilized as folk medicine in Uygur medication ( $\mathrm{Hu}$ et al. 2018). Fritillaria species like F. cirrhosa, F. verticillata and $F$. thunbergii are utilized as cough remedies in conventional Chinese prescriptions (Da-Cheng et al. 2013). Table 1 shows some of the ordinarily utilized species of genus Fritillaria with their folk uses.

\section{Phytochemicals Alkaloids}

In excess of 120 alkaloids have been isolated from the genus Fritillaria (Xiao et al. 2007). The significant phytochemicals in Fritillaria species are reported as isosteroidal alkaloids: ebeiedine, ebeienine, ebeiedinone, verticinone, imperialine, verticine, hupehenine and isoverticine. However, quantity and kind of isosteroidal alkaloids differ in numerous Fritillaria species, and clinical results can likewise be unique (Li et al. 2000). The structures of these alkaloids are given in Fig. 1.

Steroidal alkaloids have been reported in Fritillaria camtschatcensis (Japanese name kuroyuri), such as anrakorinine, veraalkamine, camtschatcanidine (Kaneko et al. 1981a), hapepunine, solasodine, tomatidenol (Kaneko et al. 1981b), solanidine (1) (Mitsuhashi et al. 1969; Kaneko et al. 1981b) and kuroyurinidine (2) (Sashida et al. 1989; Mimaki and Sashida 1990a, b); solanidine (1) has additionally been reported in F. thunbergii (Kitajima et al. 1982a). From Fritillaria imperialis bulbs, ebeinone, a steroidal base, has been isolated (Sener 1994; Farooq et al. 1994), along with the structures of forticine (4) and impericine (3) that have been displayed by spectroscopic studies. Other phytochemicals extracted from $F$. imperialis include cevanine-type alkaloids-impericine (3) and forticine (4), three steroidal alkaloids-delavine (5), imperialine (7) and persicanidine A (6) (Akhtar et al. 2002), three steroidal bases, dihydroimpranine (8), impranine (9), fetisinine (10) and an alkaloid, korsevine (11) (Akhtar et al. 2003). In F. cirrhosa, the significant alkaloids in particular imperialine (7), verticinone (12), verticine (13), ebeiedine (14), ebeiedinone (15) and chuanbeinone (16) ( $\mathrm{Li}$ et al. 1992; Wang et al. 2011) were identified; however, their amounts were low (Li et al. 1999). Furthermore, alkaloids like sinpeinine A (17), imperialine-3- $\beta$-glucoside (18), imperialine (7) and $3 \beta$-acetylimperialine have been reported from Bulbus Fritillaria Cirrhosae (BFC) (Zhang et al. 2003; Zhou et al. 2003; Lin et al. 2006a, b). In F. thunbergii, alkaloids like verticinone (12) and verticine (13) were found as the significant components, while low quantity of ebeiedine (14) and ebeiedinone (15) was known in this herb ( $\mathrm{Li}$ et al. 1999). Steroidal alkaloids-dongbeinine (19), dongbeirine (20), zhebeinine (21), peimine (22), peiminine, verticine (13) and isoverticine (23) (Wu et al. 2018) have been identified from $F$. thunbergii Miq. var. chekiangensis (Zhang et al. 1993b). In the same context, isosteroidal alkaloids are major phytochemicals detailed of which peiminine and peimine (22) are two principle alkaloid constituents (Morimoto and Kimata 1960; Li et al. 1992; Cheng et al. 2008). Moreover, some other alkaloid constituents including zhebeinine (21) (Zhang et al. 1993c), zhebeinone (24) (Zhang et al. 1992), ebeiedine (14) (Kim et al. 2016), puqiedine (8) (Zhou et al. 2010), N-demethylpuqietinon (Zhou et al. 2017), eduardinine (25) (Suh et al. 2018), ebeiedinone (15) (Wu et al. 2018), puqiedinone (Zhou et al. 2010), eduardine (26), zhebeirine (27) (Zhang et al. 1991), 3 $\beta$-hydroxy- $5 \alpha$-jervanin-12-en-6-one (28) (Suh et al. 2018), frithunbol A (29), frithunbol B (30) (Suh et al. 2018) were also identified. The investigation on the flower of $F$. thunbergii has reported eight components as main chemical constituents (Peng et al. 2012): 
Table 1 Commonly used species of genus Fritillaria with their folk uses

\begin{tabular}{|c|c|c|c|c|}
\hline S. No. & Species & Local name & Folk uses & References \\
\hline 1 & Fritillaria ebeiensis & - & $\begin{array}{l}\text { High antitussive and expectorant effects. A } \\
\text { crude drug Ebeibeimu is prepared by treating } \\
\text { its bulbs with lime and then bleached in sun, } \\
\text { serves as a substitute drug for Beimu used } \\
\text { for moisturizing lungs, clearing heat, treating } \\
\text { cough, asthma and tracheitis like throat } \\
\text { diseases }\end{array}$ & Yu et al. (1985); Li et al. (1994), Wu et al. (1995) \\
\hline 2 & Fritillaria cirrhosa & Chuanbeimu & $\begin{array}{l}\text { Used to treat cough and asthma, Bulbs can be } \\
\text { used as remedies for clearing the lungs of } \\
\text { phlegm and cooling heat }\end{array}$ & $\begin{array}{l}\text { Bensky et al. (2004); Bensky and Gamble (1993); } \\
\text { Li et al. (2006a, b, 2009), Konchar et al. (2011), } \\
\text { Wang et al. (2011) }\end{array}$ \\
\hline 3 & Fritillaria thunbergii & Zhebeimu & $\begin{array}{l}\text { Used as antitussive, antiasthmatic and expec- } \\
\text { torant, to treat various diseases like cough, } \\
\text { eliminating phlegm, relieving pain and anti- } \\
\text { inflammatory disorders }\end{array}$ & $\begin{array}{l}\text { Qian and Xu (1985); Xiao et al. (1992); Zhou et al. } \\
\text { (2003); Peng et al. (2012) }\end{array}$ \\
\hline 4 & Fritillaria pallidiflora & Yibeimu & $\begin{array}{l}\text { Cough treatment in TCM, used as antitussive, } \\
\text { expectorant and antiasthmatic medicine }\end{array}$ & Li et al. (1993); Zhou et al. (2003) \\
\hline 5 & Fritillaria ussuriensis Maxim & Ping-Beimu & $\begin{array}{l}\text { Used to treat swollen throat and lung ailments } \\
\text { in Chinese medicine }\end{array}$ & Perry (1980) \\
\hline 6 & Fritillaria maximowiczii & - & Used to treat cough & - \\
\hline 7 & Fritillaria imperialis & - & $\begin{array}{l}\text { Treatment of various diseases like asthma, sore } \\
\text { throat, bronchitis, cough, scrofula, haem- } \\
\text { optysis, dysuria and gland tumour. Home } \\
\text { remedy for haemorrhage, cough and phlegm, } \\
\text { high fever, treatment of abscess, lack of milk, } \\
\text { eye disease, asthma, rheumatism and act as } \\
\text { antianxiety/ depression }\end{array}$ & $\begin{array}{l}\text { Bailey (1966), Perry (1980), Aydın et al. (2018), } \\
\text { Abbaszadeh et al. (2019) }\end{array}$ \\
\hline 8 & Fritillaria roylei & Kakoli & $\begin{array}{l}\text { Ayurveda drug Ashtavarga is prepared, used } \\
\text { for fever, burning sensation, phthisis and } \\
\text { broncho-asthma }\end{array}$ & Singh (2006), shaheen et al. (2014) \\
\hline 9 & Fritillaria anhuiensis & - & Used to treat asthma & Shou et al. (2009) \\
\hline 10 & Fritillaria unibracteata & - & $\begin{array}{l}\text { Used as antitussive, antiasthmatic and expecto- } \\
\text { rant agents in TCM }\end{array}$ & Liang (2004) \\
\hline 11 & Fritillaria verticillata & - & Cough remedies & Da-Cheng et al. (2013) \\
\hline 12 & Fritillaria tortifolia & - & Folk medicine in Uygur medicine & Hu et al. (2018) \\
\hline 13 & Fritillaria delavayi & - & Used antitussive and apophlegmatic & Duan et al. (2012) \\
\hline
\end{tabular}

(see figure on next page.)

Fig. 1 Structures of various alkaloids of genus Fritillaria: 1. solanidine, 2. kuroyurinidine, 3. impericine, 4. forticine, 5. delavine, 6. persicanidine A, 7. imperialine, 8. dihydroimpranine, 9. impranine, 10. fetisinine, 11. korsevine, 12. verticinone, 13. verticine, 14. ebeiedine, 15. ebeiedinone, 16. chuanbeinone, 17. sinpeinine A, 18. imperialine-3- $\beta$-glucoside, 19. dongbeinine, 20. dongbeirine, 21. zhebeinine, 22. peimine, 23. isoverticine, 24. zhebeinone, 25. eduardinine, 26. eduardine, 27. zhebeirine, 28. 3ß-hydroxy-5a-jervanin-12-en-6-one, 29. frithunbol A, 30. frithunbol B, 31. yibeinine, 32. yubeinine, 33. hupehenine, 34. yibeinoside, 35. imperialine-3ß-D-glucoside, 36. (20R,22R,23R,25R)- 3b,23-dihydroxy-N-methyl-veratram-13(17)- en-6-one 37. sipeimine, 38. ebeiensine, 39-42. yibeinones A-D, 43. peimisine, 44. imperialine- $\beta-\mathrm{N}$-oxide, 45. isoverticine- $\beta-\mathrm{N}$-oxide, 46. yibeissine, 47. yibeinoside $\mathrm{B}$, 48. ebeinine, 49. ebeienine, 50. 22S, 25S,5a-Vertram

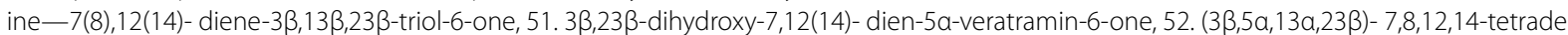
hydro-5,6,12,13-tetrahydro-3,23-dihydroxyveratraman-6-one, 53. (3ß,5a,13a,23ß)- 7,8,12,14-tetradehydro-5,6, 12,13-tetrahydro-3,13,23-trihydroxyv eratraman-6-one 54.3-O-acetoxyverticinone 55. 3-O-acetylverticine, 56. pingbeinine, 57. pingbeininoside, 58. ussurienine, 59. pingbeimunone $A$,

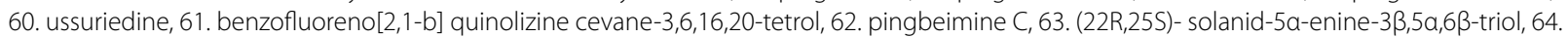
delafrinone, 65. delafrine, 66. ningpeisine, 67. delavine 3-O- $\beta$-D-glucopyranoside, 68. persicanidine B 3-O- $\beta$-D-glucopyranoside, 69. persicanidine

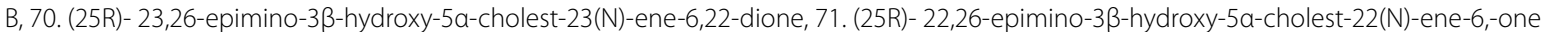
3-O- $\beta$-D-glucopyranoside, 72. (25R)- 23,26-epimino-3 $\beta$-hydroxy-5a-cholest-23(N)- ene-6,22-dione3-O- $\beta$-Dglucopyranoside, 73. (20R,25R)- 23,26-epi

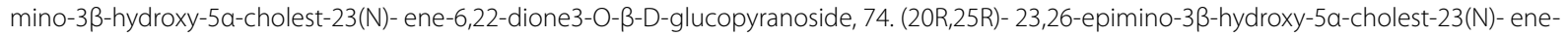

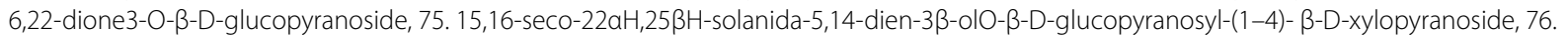
23-isokuroyurinidine 77. hapepunne 3-O- $\beta$-cellobioside, 78. siechuansine, 79. taipaienine, 80. puqienine A, 81. puqienine B, 82-84. puqienines $\mathrm{C}-\mathrm{E}$, 85. puqienine $\mathrm{F}, 86 . \mathrm{N}$-dimethylpuqietinone 87. puqietinonoside 88. puqietinone, 89. puqietinedione, $90.3 \mathrm{a}-$ puqiedin-7-ol, 91. lichuanine,

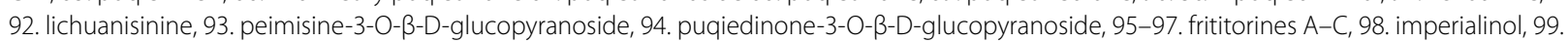
peimisine-3-O- $\beta$-D-glucoside, 100. imperialine-3-O- $\beta$-D-glucoside, 101. delavinone, 102. hupehenizioiside 


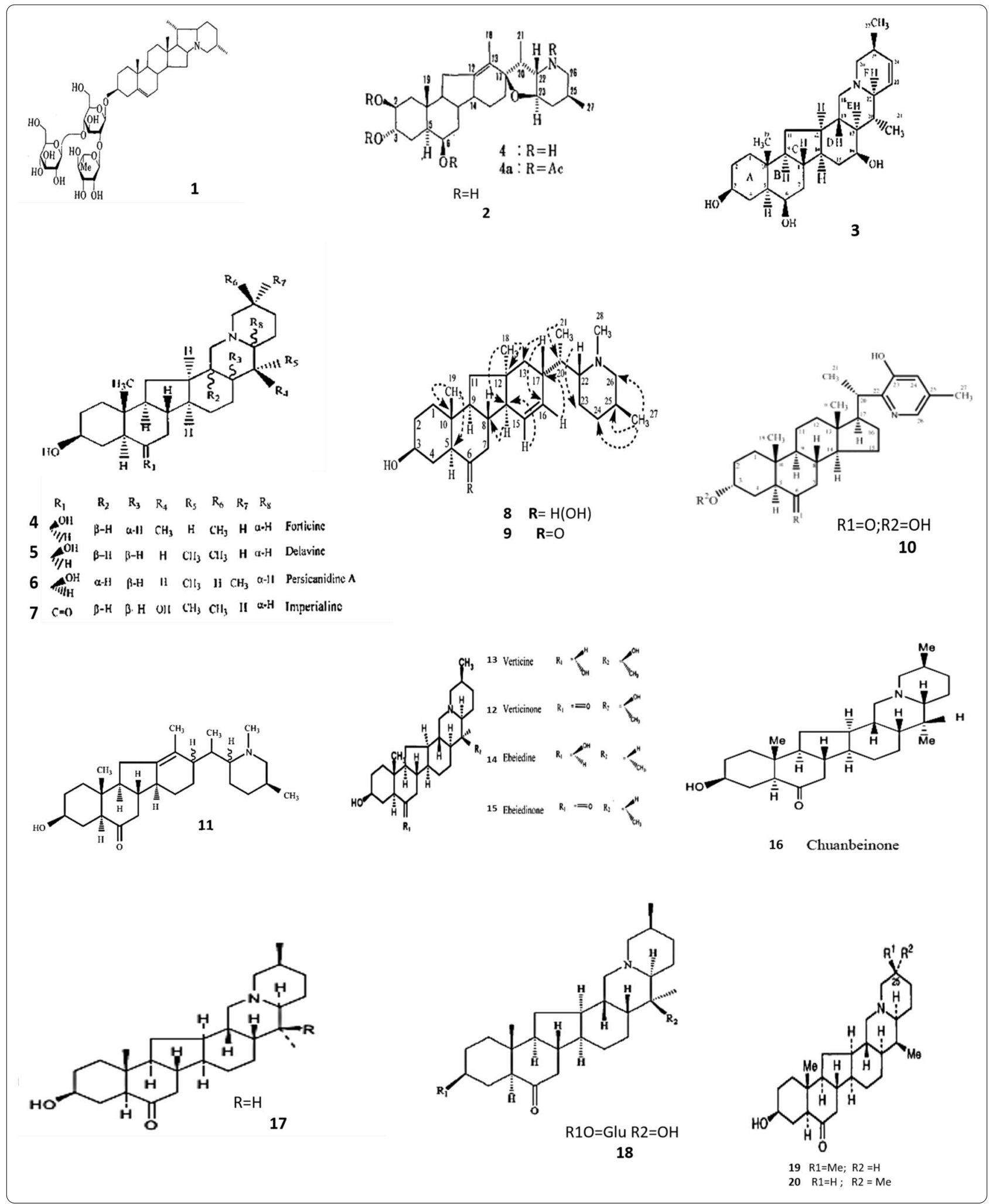




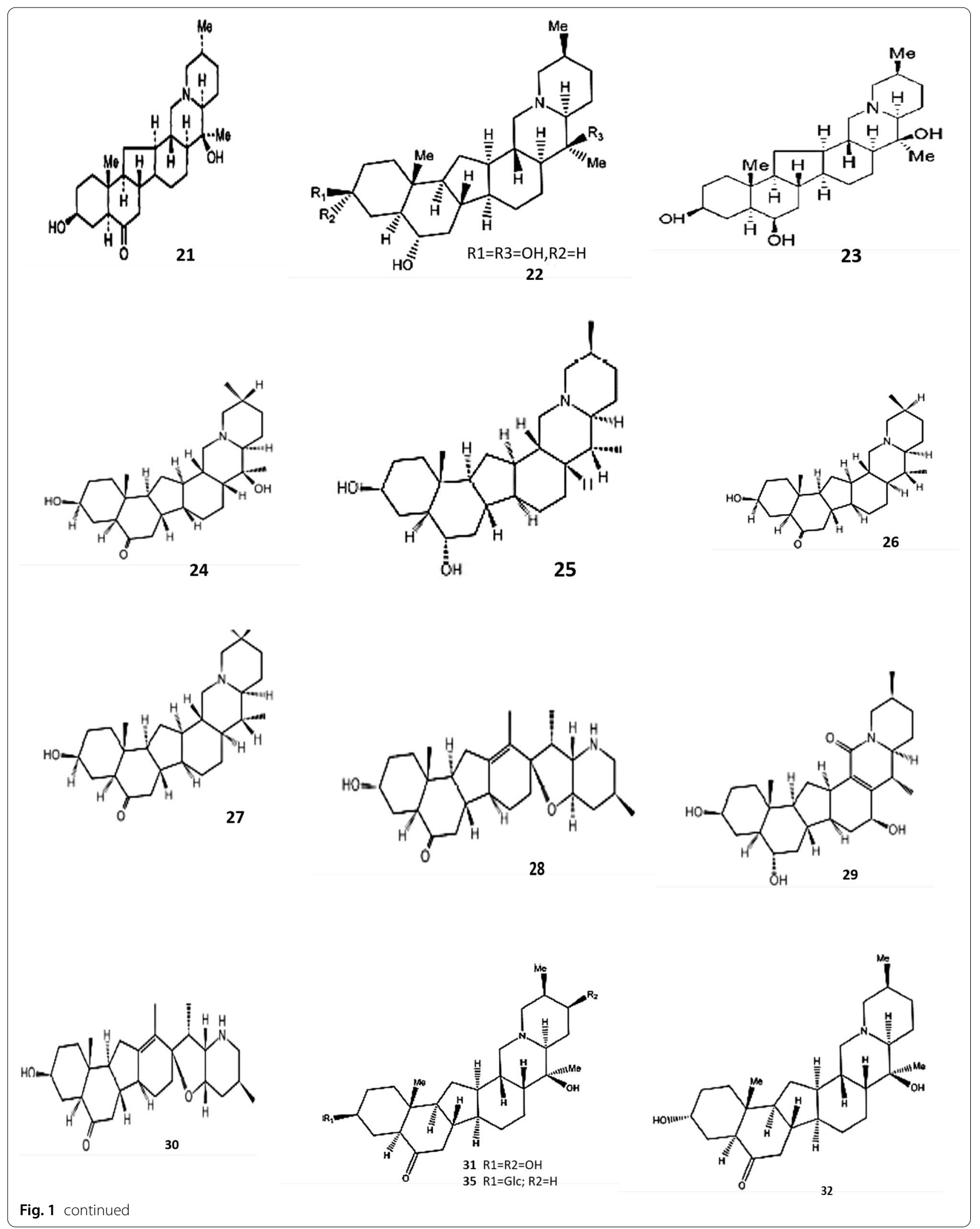




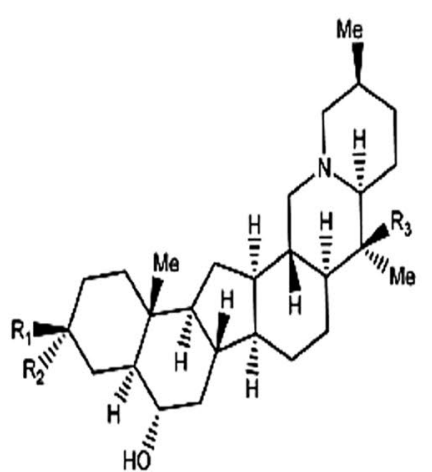

$33 \mathrm{R} 1=\mathrm{R} 3=\mathrm{H} ; \mathrm{R} 2=\mathrm{OH}$
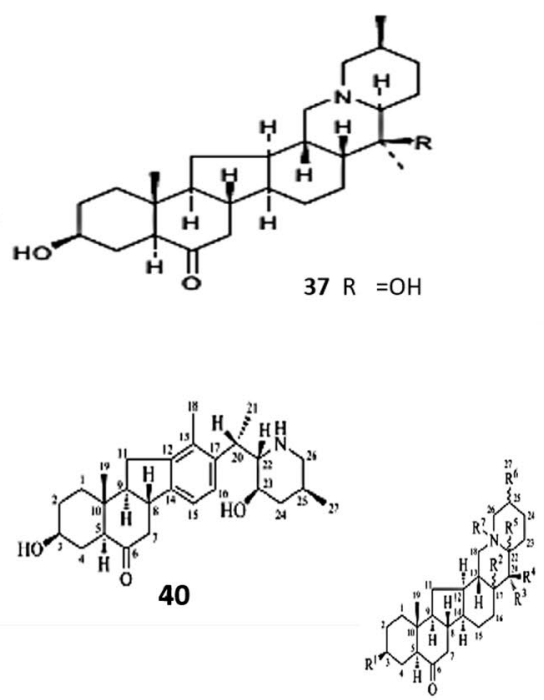

$\begin{array}{lllllll}R^{1} & R^{2} & R^{3} & R^{4} & R^{5} & R^{6} & R^{7}\end{array}$

OH $a \cdot$ H Me OH $\beta$.H $a \cdot M e$.

OGlu $\alpha$-H OH Mc B.H B.Me - 42
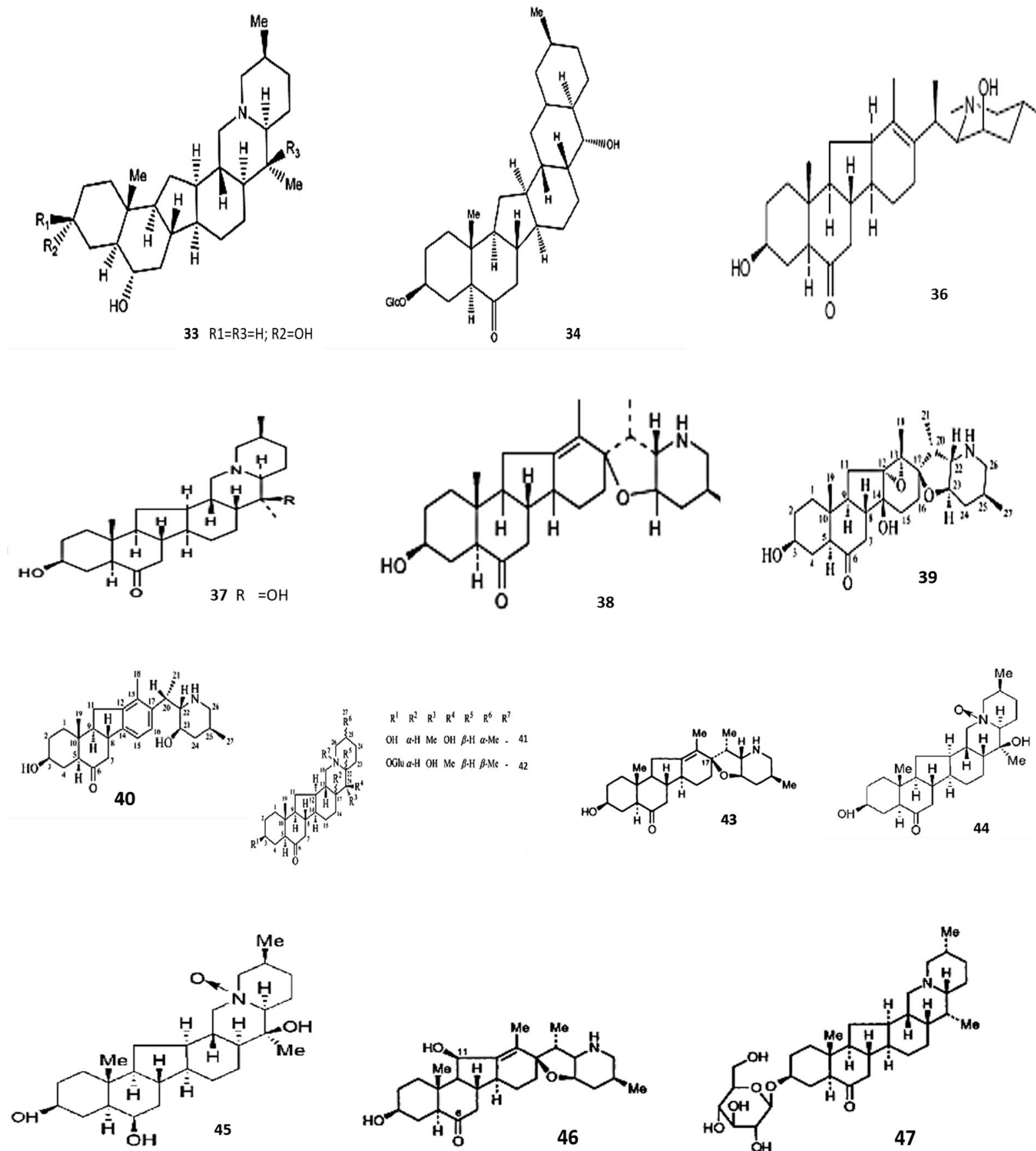

Fig. 1 continued

(1) 1-heptadecanol $\left(\mathrm{C}_{17} \mathrm{H}_{36} \mathrm{O}\right)$;

(2) monoheptadecanoin $\left(\mathrm{C}_{20} \mathrm{H}_{40} \mathrm{O}_{4}\right)$;

(3) 5,7-dihydroxy-2-(4-hydroxy-3-methoxyphenyl)-3methoxy-4H-chromen-4-one $\left(\mathrm{C}_{17} \mathrm{H}_{14} \mathrm{O}_{7}\right)$;

(4) isorhamnetin $\left(\mathrm{C}_{16} \mathrm{H}_{12} \mathrm{O}_{7}\right)$;

(5) dihydroapigenin $\left(\mathrm{C}_{15} \mathrm{H}_{12} \mathrm{O}_{5}\right)$;

(6) kaempferol-3-O- $\alpha$-l-rhamnoside $\left(\mathrm{C}_{21} \mathrm{H}_{20} \mathrm{O}_{10}\right)$;
(7) kaempferol-3-O- $\alpha$-l-glucoside $\left(\mathrm{C}_{21} \mathrm{H}_{20} \mathrm{O}_{11}\right)$;

(8) kaempferitrin $\left(\mathrm{C}_{27} \mathrm{H}_{30} \mathrm{O}_{14}\right)$.

Phytochemical investigation on Fritillaria pallidiflora has revealed the isolation of steroidal alkaloids (Xu et al. 1993; Li et al. 2002) and nonsteroidal alkaloids. Steroidal alkaloids like yibeinine (31) (Xu et al. 2014), yubeinine (32) (Zhang et al. 1993d), peimine (22) (Li and Wu 1986), 


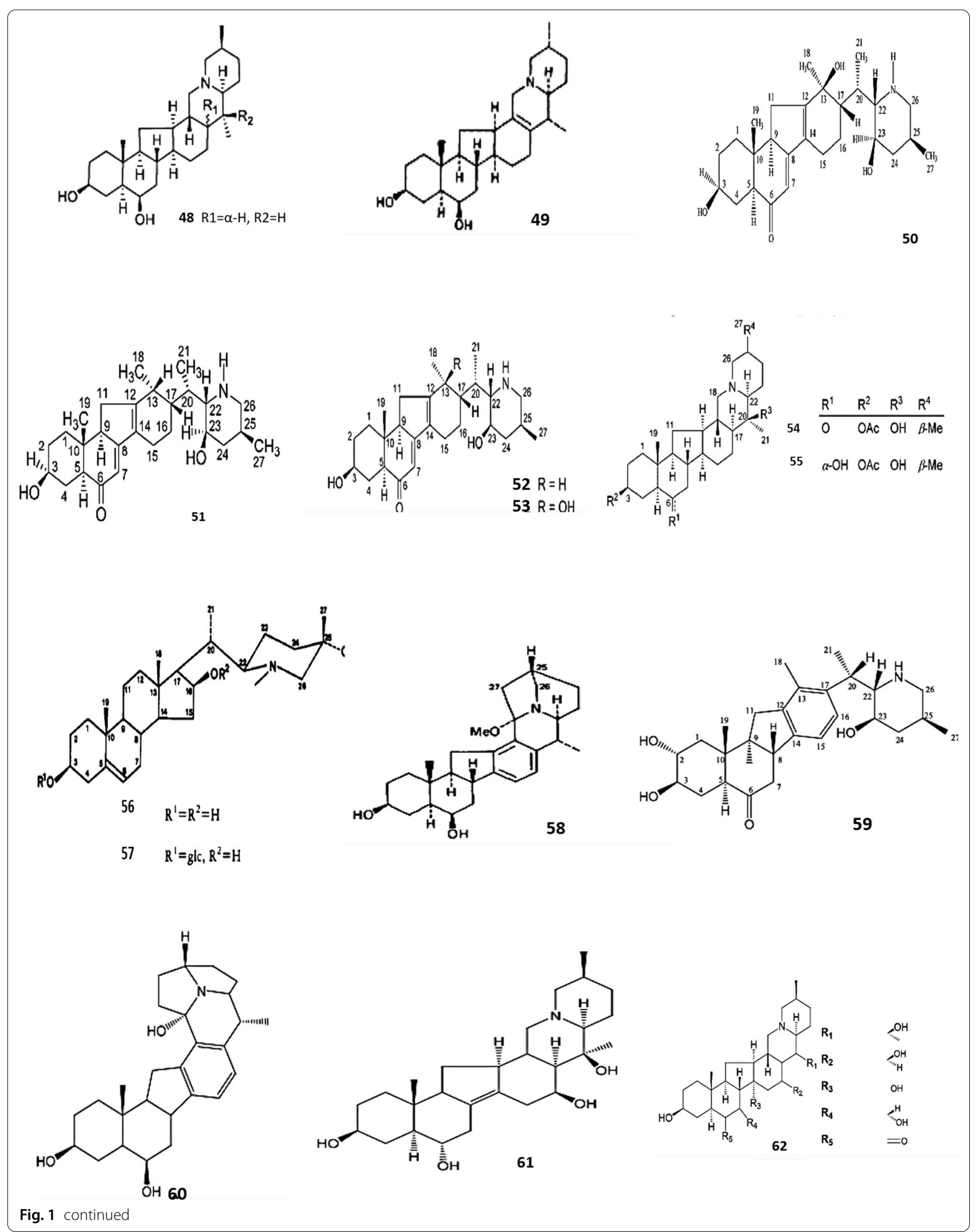



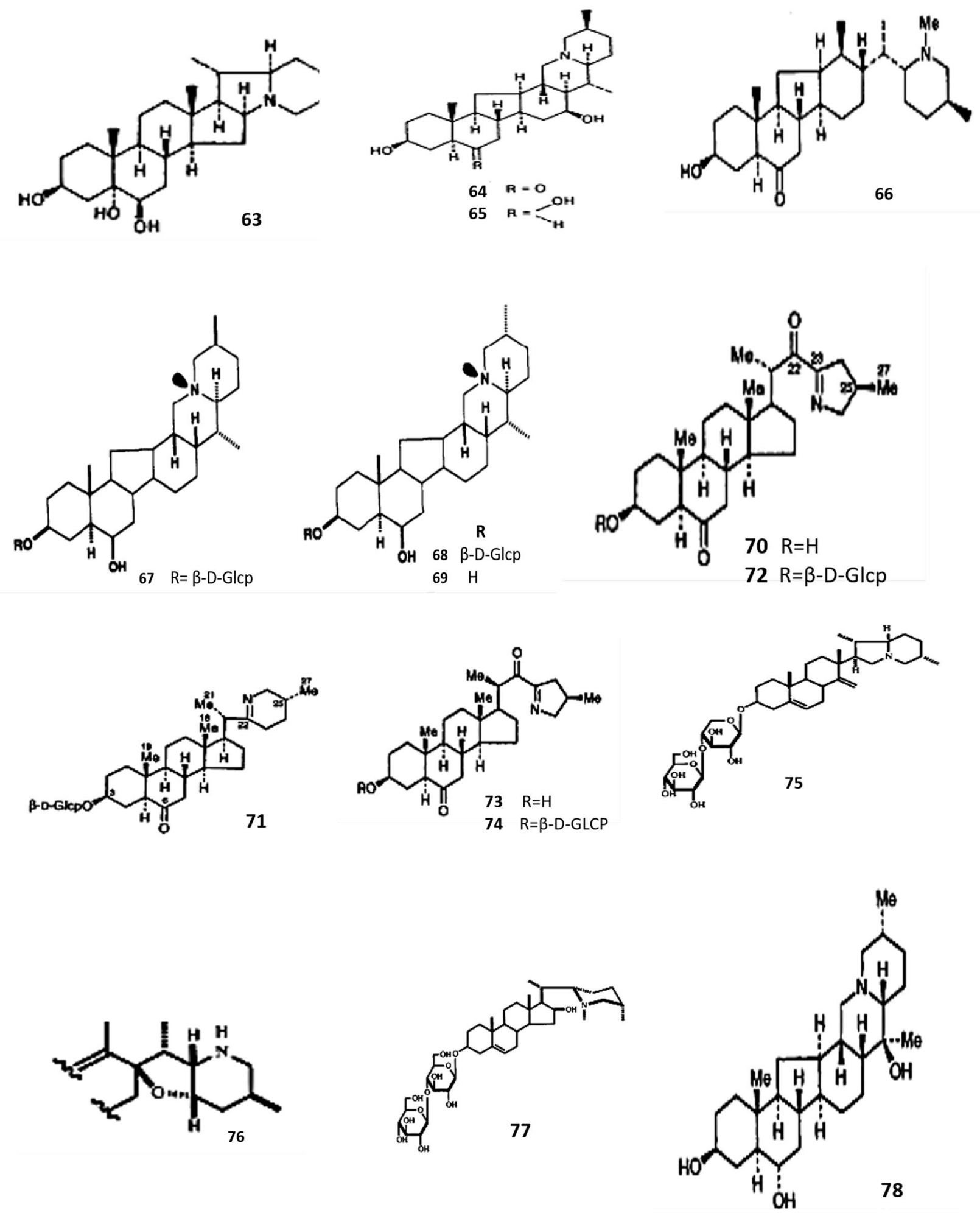

Fig. 1 continued 

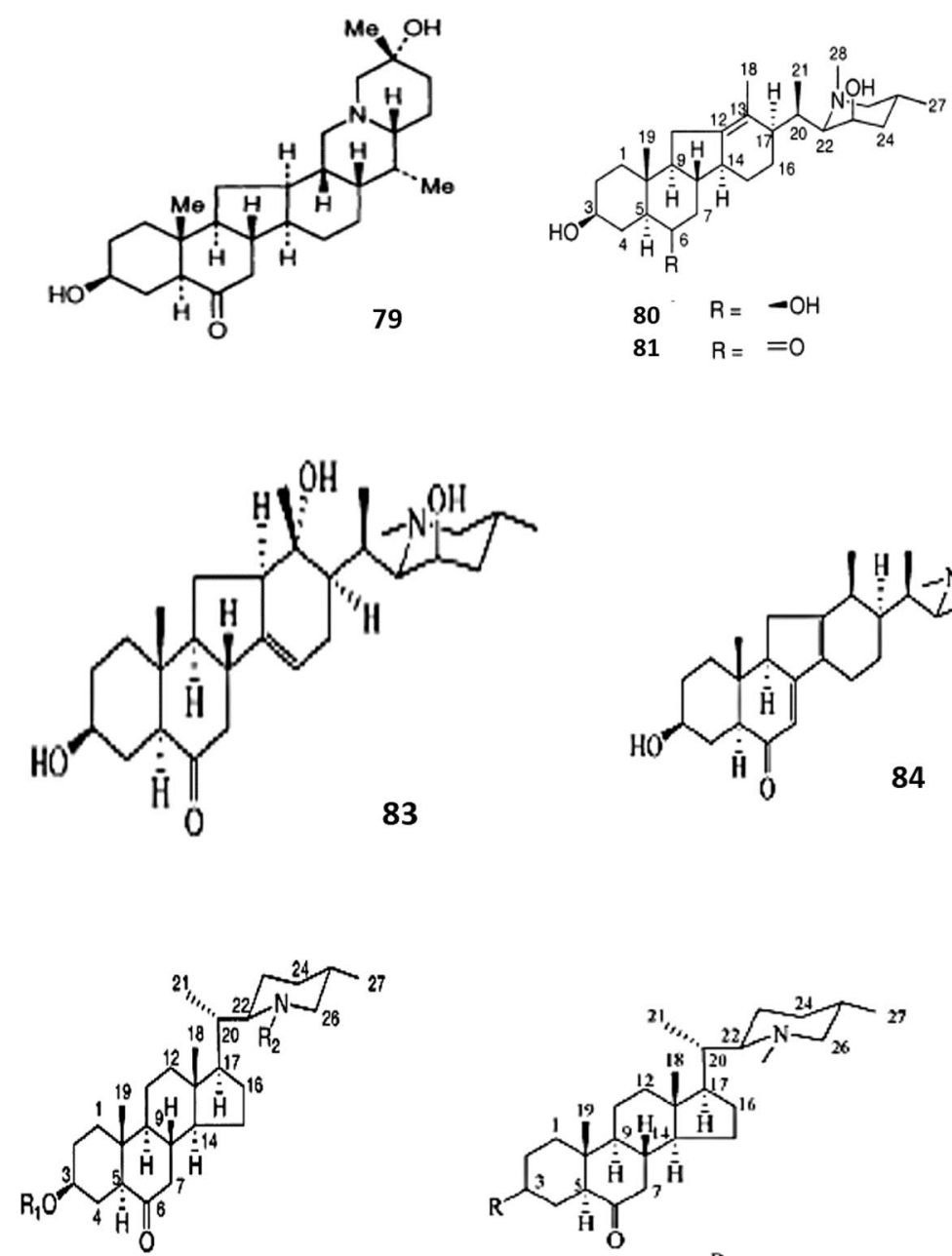

$86 \quad R_{1}=H \quad R_{2}=H$

$87 \quad R_{1}=$ Glu $R_{2}=$ Me

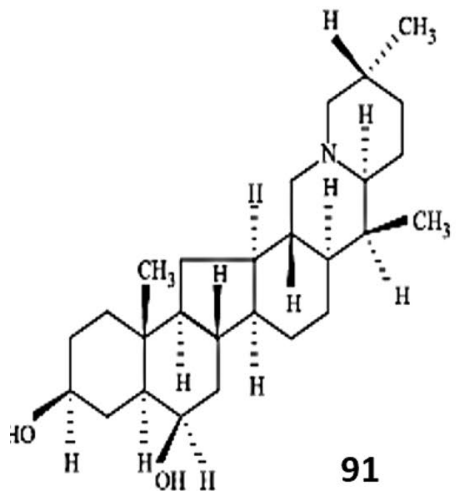

Fig. 1 continued

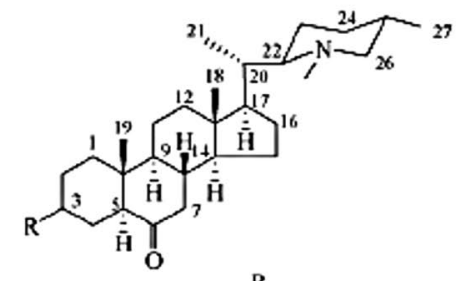

$$
\begin{array}{ll}
89 & \mathrm{R} \\
88 & -\mathrm{O} \\
& -\mathrm{OH}
\end{array}
$$

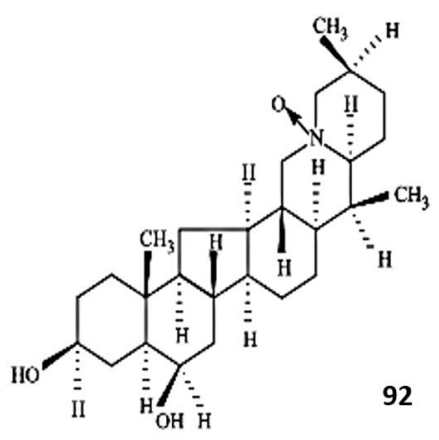

92
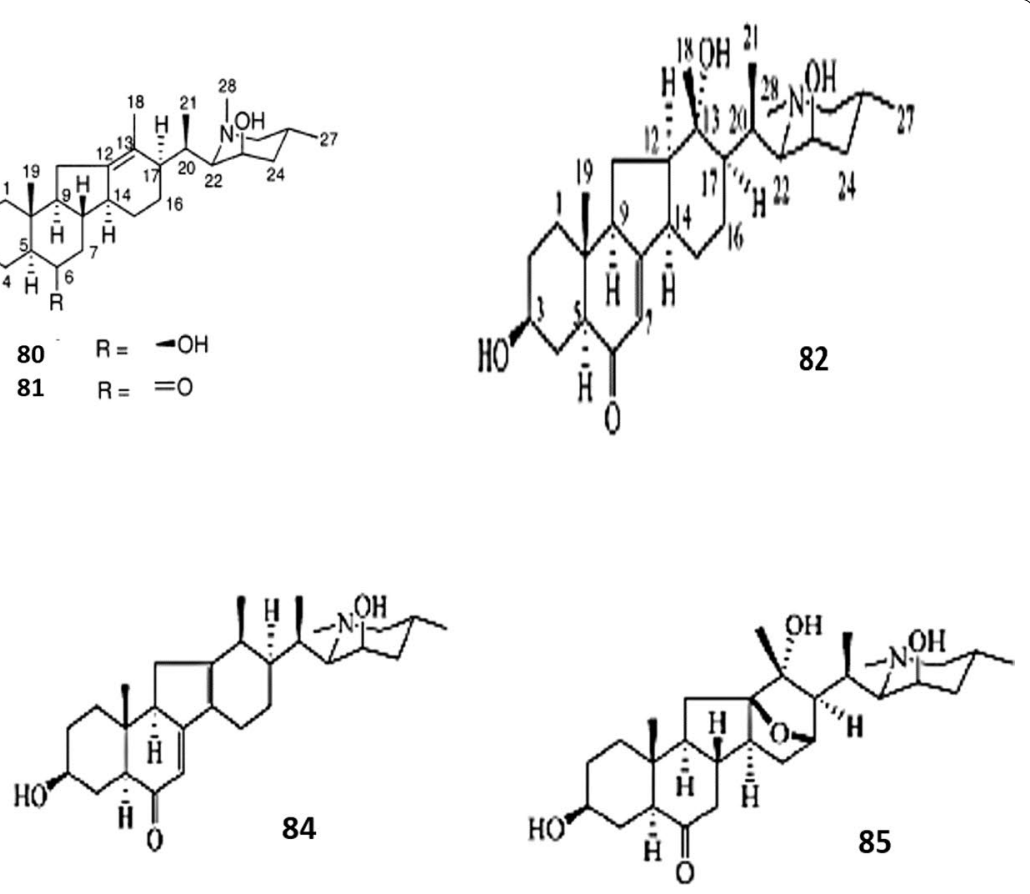

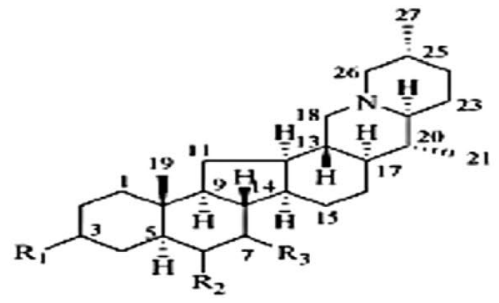

$\begin{array}{lll}R_{1} & R_{2} & R_{3}\end{array}$

...

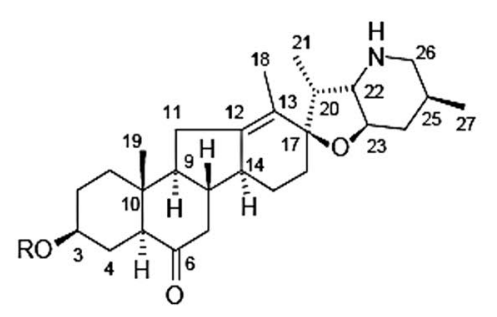

$93 \mathrm{R}=\beta$-D-glc 


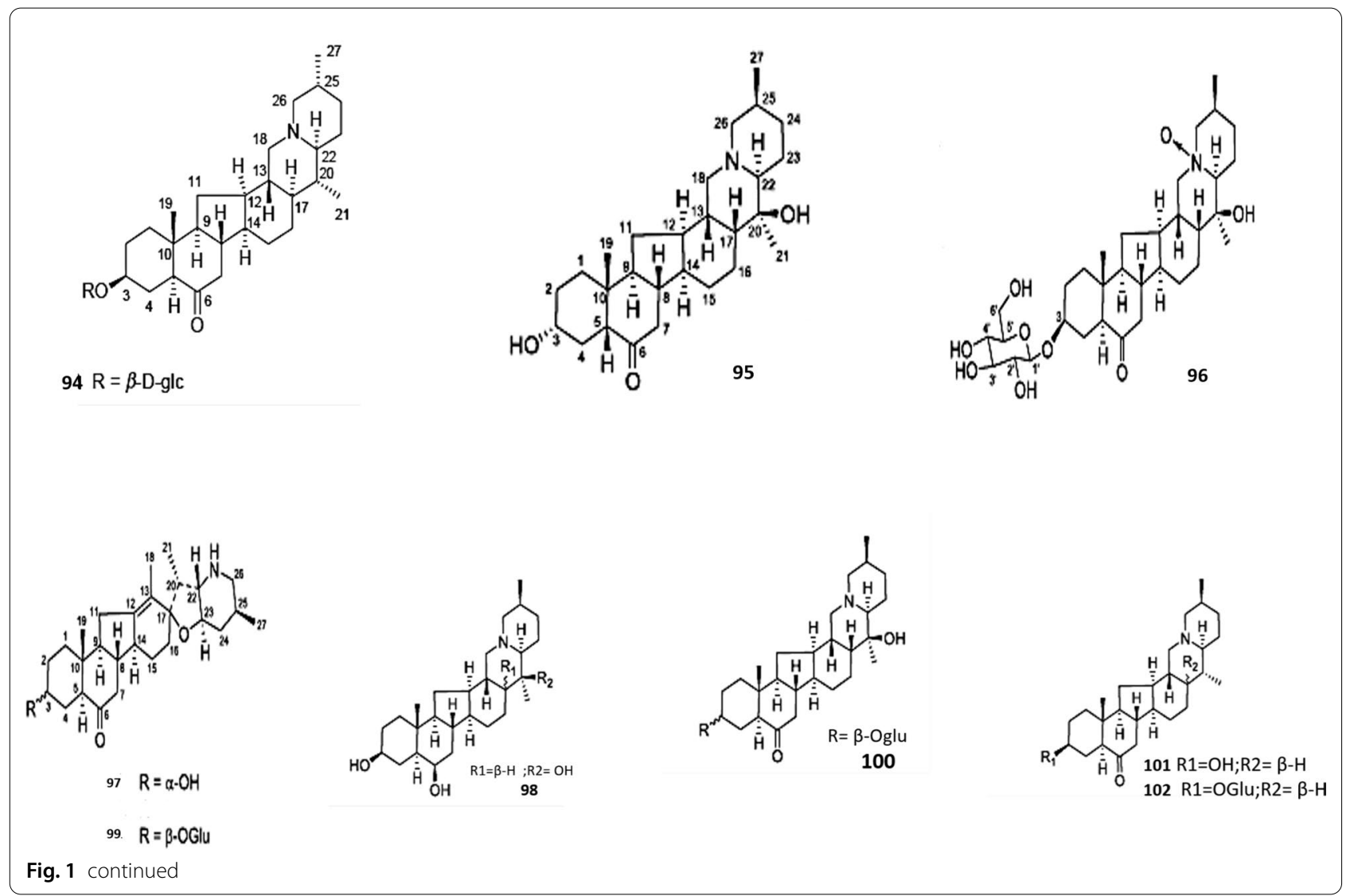

hupehenine (33), yibeinoside (34) (Xu et al. 1990a, b) imperialine-3 $\beta$-D-glucoside (35) (Xu et al. 1990a, b), imperialine (7) (Akhtar et al. 2002), (20R,22R,23R,25R)3b,23-dihydroxy-n-methyl-veratram-13(17)- en-6-one (36) (Shen et al. 2012b), sinpeinine A (17) (Liu et al. 1984), sipeimine (37) (Akhtar et al. 2002), ebeiensine (38) (Zhang et al. 2011), yibeinones A-D (39-42) (Li et al. 2016), dongbeinine (19) (Zhang et al. 1993b), chuanbeinone (16), imperialine- $\beta$-N-oxide (44) (Chen et al. 2004), isoverticine (23) and isoverticine- $\beta-\mathrm{N}$-oxide (45) (Wang et al. 2015) were studied and identified. Investigations have revealed fifteen more isosteroidal alkaloids including ten cevanetype ones (Xu et al. 1990a, 1993; Liu et al. 1984, Xu et al. 2014) four jervine-type ones (Xu et al. 1992) and a veratramine-type alkaloid (Shen et al. 2012b; Hao et al. 2013). Phytochemical investigations on F. pallidiflora have also reported that the free isosteroidal alkaloid imperialine (7)

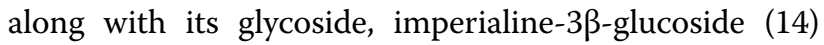
were the principal constituents (Li et al. 1999; 2002). Steroidal alkaloids, yibeissine (46), yibeinoside B (47), yibeinoside $C$ and yibeinine-3-O- $\beta-D$ glucopyranoside have been reported (Xu et al. 1992, 1993). From Fritillaria ebeiensis generally found in China, C-nor-D-homo steroidal alkaloids were reported like peimine (22), peiminine, verticinone (12), verticine (13), ebeinine (48), ebeinone, ebeiensine (38) and hupehenidine (Wu et al. 1989), ebeiedine (14) (Li et al. 1995a, b). Seven alkaloids were identified from Fritillaria ebeiensis var. purprea including ebeienine (49), ebeiedine (14), ebeiedinone (15), verticine (13), verticinone (12) and isoverticine (23) (Lee et al. 1988; Li et al. 1995a, b) as well as steroidal alkaloid ebeietinone, was isolated (Ping et al. 1992). F. hupehensis, clinically, the most toxic Fritillaria species from which hupehenine (33) (Li et al. 1990b), a veratramine alkaloid to be specific $22 \mathrm{~S}, 25 \mathrm{~S}, 5 \alpha$-vertramine-7(8),12(14) - diene-3 $\beta, 13 \beta, 23 \beta$ -

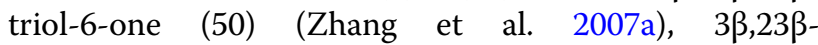
dihydroxy-7,12(14)- dien-5 $\alpha$-veratramin-6-one (Zhang et al. 2007b) ebeinine (48) and zhebeinine (21) were reported and four penta and hexacyclic cevan-veratraman-based steroidal alkaloids, were isolated with structure of compound 1 was explained as 3-O-acetylverticine (55), compound 2 as $(3 \beta, 5 \alpha, 13 \alpha, 23 \beta)-7,8,12,14$-tetradehydro-5,6,12,13-tetrahydro-3,23-dihydroxyveratraman-6-one (52), compound 3 as 3-O-acetoxyverticinone (54) and 4 which was clarified as $(3 \beta, 5 \alpha, 13 \alpha, 23 \beta)$ 7,8,12,14-tetradehydro-5,6,12,13-tetrahydro-3,13,23-trihydroxyveratraman-6-one (53) (Zhang et al. 2008a). Phytochemical examination on leaves of Fritillaria 
ussuriensis Maxim has reported isolation of steroidal alkaloids specifically pingbeinine (56) and pingbeininoside (57) (Xu et al. 1990c). Additionally, cevanine-type alkaloid ussurienine (58), solanidine (1), verticinone (12) (Wang et al. 2015), imperialine (7), isoverticine (23) (Pae et al. 2002; Oh et al. 2003; Wang et al. 2015), pingbeimunone A (59) (Yang and Duan 2012), ussuriedine (60) (Kitamura et al. 1989a), benzofluoreno[2,1-b]quinolizine cevane-3,6,16,20-tetrol (61) (Kitamura et al. 1989b), ebeiedinone (15) (Lee et al. 1988), pingbeimine C (62) (Xu et al. 1990c, d), verticine (13) (Kaneko et al. 1980; Oh et al. 2003; Wang et al. 2015) and peimisine (43) (Oh et al. 2003; Wang et al. 2015) have been identified from Fritillaria ussuriensis (Ito et al. 1976; Kaneko et al. 1981b, Lee et al. 1988; Kitamura et al. 1988). Phytochemical examination on Fritillaria delavayi has detailed three alkaloids specifically a natural solanidanine $(22 \mathrm{R}, 25 \mathrm{~S})$ - solanid- $5 \alpha$-enine$3 \beta, 5 \alpha, 6 \beta$-triol (63) and two cevanine-type delafrinone (64) and delafrine (65) (Kaneko et al. 1988). From Fritillaria ningguoensis, alkaloids ningpeisine (66) (N-methy13/3-hydroxy-saveratranine-6-one), peimine (22), peiminine, isoverticine (23) and peimisine (43) were identified (Li et al. 1988). On the other hand, from F. persica bulbs, pyrrolidine and piperidine side-chain alkaloids have been isolated (Ori et al. 1992b), alongside five cerveratrum alkaloids, namely delavine (5), persicanidine A (6) (Kaneko et al.1985; Ori et al. 1992a), persicanidine B 3-O- $\beta$-D-glucopyranoside (68), delavine 3-O- $\beta$-Dglucopyranoside (67) and persicanidine B (69) (Ori et al. 1992b) as well as five steroidal alkaloids, with structures revealed as (25R)- 22,26-epimino-3 $\beta$-hydroxy- $5 \alpha$-cholest$22(\mathrm{~N})$-ene-6,-one 3-O- $\beta$-D-glucopyranoside (71), (25R)23,26-epimino-3 $\beta$-hydroxy- $5 \alpha$-cholest-23(N)-ene-6,22dione (25R)- 23,26-epimino-3 $\beta$-hydroxy-5a-cholest-23(N)- ene6,22-dione3-O- $\beta$-Dglucopyranoside (72), (20R,25R)23,26 -epimino-3 $\beta$-hydroxy- $5 \alpha$-cholest- $23(\mathrm{~N})$ - ene-6,22dione3-O- $\beta$-D-glucopyranoside (74) and (20R,25R)- 23,26-epimino-3 $\beta$-hydroxy- $5 \alpha$-cholest-23(N)ene-6,22-dione3-O- $\beta$-D-glucopyranoside (73), were reported (Ori et al. 1992c). Phytochemical examination on $F$ maximowiczii bulbs has reported alkaloids like 15,16-seco- $22 \alpha \mathrm{H}, 25 \beta \mathrm{H}$-solanida-5,14-dien-3 $\beta$-olO- $\beta$ - $\mathrm{D}$ glucopyranosyl-(1-4)- $\quad \beta$-D-xylopyranoside $\quad$ (75), 23 -isokuroyurinidine (76), hapepunne $3-\mathrm{O}-\beta$-cellobioside (77) and including a jerveratrinalkaloid, kuroyurinidine (2) (Qian and Nohara 1995). In F. siechuanica found in Sichuan region of China, cevanine-type steroidal alkaloid, siechuansine (78), peimisine (43) and imperialine (7) have been identified (Wang et al. 1992a). During phytochemical analysis on Fritillaria taipaiensis L. var. ningxiaensis, Hu et al. 1993 identified C-nor-D-homo-steroidal alkaloid specifically taipaienine (79), chuanbeinone (16), imperialine (7), verticinone (12), perimissine and isovertisine. Steroidal alkaloids puqiedinone (Lin et al. 1995), puqienine $A(80)$, puqienine $B$ (81) (Li et al. 2006b), puqienines C-E (82-84) (Jiang et al. 2006), puqienine F (85), $\mathrm{N}$-dimethylpuqietinone (86), puqietinonoside (87), puqietinone (88), (Jiang et al. 2005) puqietinedione (89), $3 \alpha-$ puqiedin-7-ol (90), puqiedine (8) (Jiang et al. 2006) and peimisine (43) (Wang et al. 1992) were identified from $F$. puqiensis (Li et al. 1990a; Lin et al. 1995). From Fritillaria roylei alkaloids peiminine, peimine (22), peimisine (43), peimiphine, peimitidine and peimidine were isolated (Singh 2006). Fritillaria lichuanensis P. Li et C.P. Yang is another species from genus Fritillaria endemic to northwest area of Hubei region, China; its phytochemical examination has revealed two new C-nor-D-homosteroidal alkaloids lichuanisinine (92) and lichuanine (91) (Pi et al. 2006a, b). Phytochemical investigation has reported steroidal alkaloids from $F$. unibracteata bulbs specifically peimisine (43), peimisine-3-O- $\beta$-D-glucopyranoside (93), puqiedinone-3-O- $\beta$-D-glucopyranoside (94) (Zhang et al. 2011), puqiedine (8) (Jiang et al. 2006) and puqiedinone (Lin et al. 1995). From Bulbs of Fritillaria wabuensis, imperialine (7), isoverticine (23), imperialine- $\beta-\mathrm{N}$-oxide (44), isoverticine- $\beta$-N-oxide (45) (Wang et al. 1992, 2012) were isolated. On Fritillaria tortifolia X. Z. Duan et X. J. Zheng, phytochemical examination has reported isosteroidal alkaloids, frititorines A-C (95-97) ( $\mathrm{Hu}$ et al.2018), imperialinol (98) (Choudhary et al. 1998), peimisine (43) (Wang et al. 1992), peimisine-3-O- $\beta$-Dglucoside (99) (Zhang et al. 2011), ebeinine (48) (Wu et al. 1989), imperialine (7) (Kaneko et al. 1985), yubeinine (32) (Zhang et al. 1993d), imperialine-3-O- $\beta$-D-glucoside (100) (Huang et al. 1990), ebeiedinone (15), delavinone (101) (Lin et al.1995) and hupehenizioiside (102) (Pi et al. 2006a, b). Compound frititorines C (97) is a jervine-type alkaloid, and imperialinol (98) is another natural cevanine-type alkaloid. Table 2 shows different alkaloids from Fritillaria along with their uses.

\section{Terpenoids}

Terpenoids have been reported as second significant and important chemical constituents in Genus Fritillaria. The structures of these terpenoids are given in Fig. 2. Ten novel diterpenoids, namely fritillebinides A, B and C, fritillebin A, fritillebin $B$, fritillcbin $C$, fritillebin $D$, fritillebin $\mathrm{R}$, fritillebinol and fritillebic acid, have been accounted as non-basic constituents of Fritillaria ebeiensis (Wu et al. 1995), along with a kaurane diterpenes ent3 $\beta$-butanoyloxykaur-15-en-17-ol; two labdane diterpenes with structure 6-oxo-2 $\alpha$-hydroxy-labda-7,12(E), 14-triene (104) and $6 \alpha, 7 \beta$-dihydroxy-labda-8 (17),12(E),14-triene (103) were identified from F. ebeiensis. Structure of five 


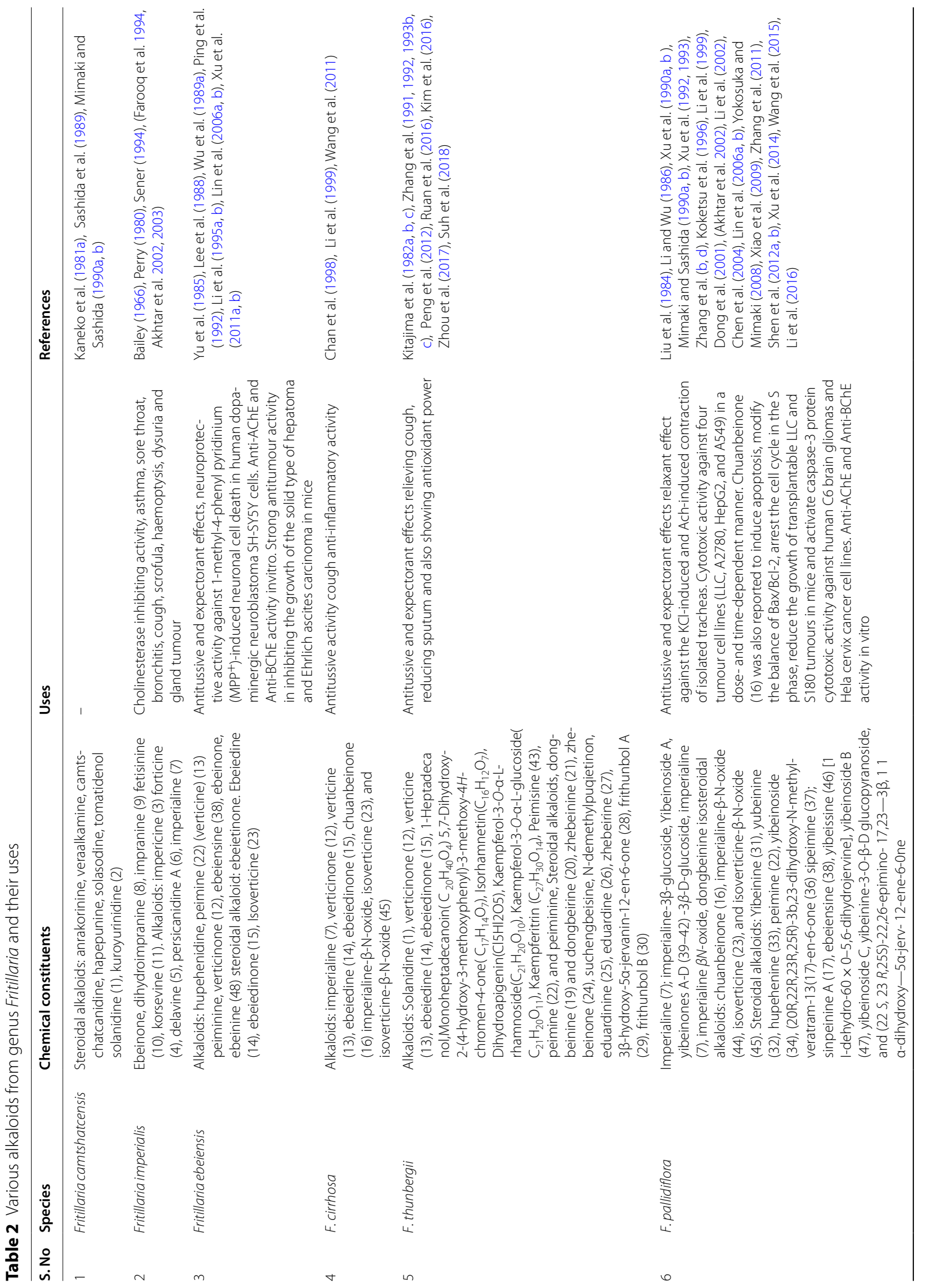




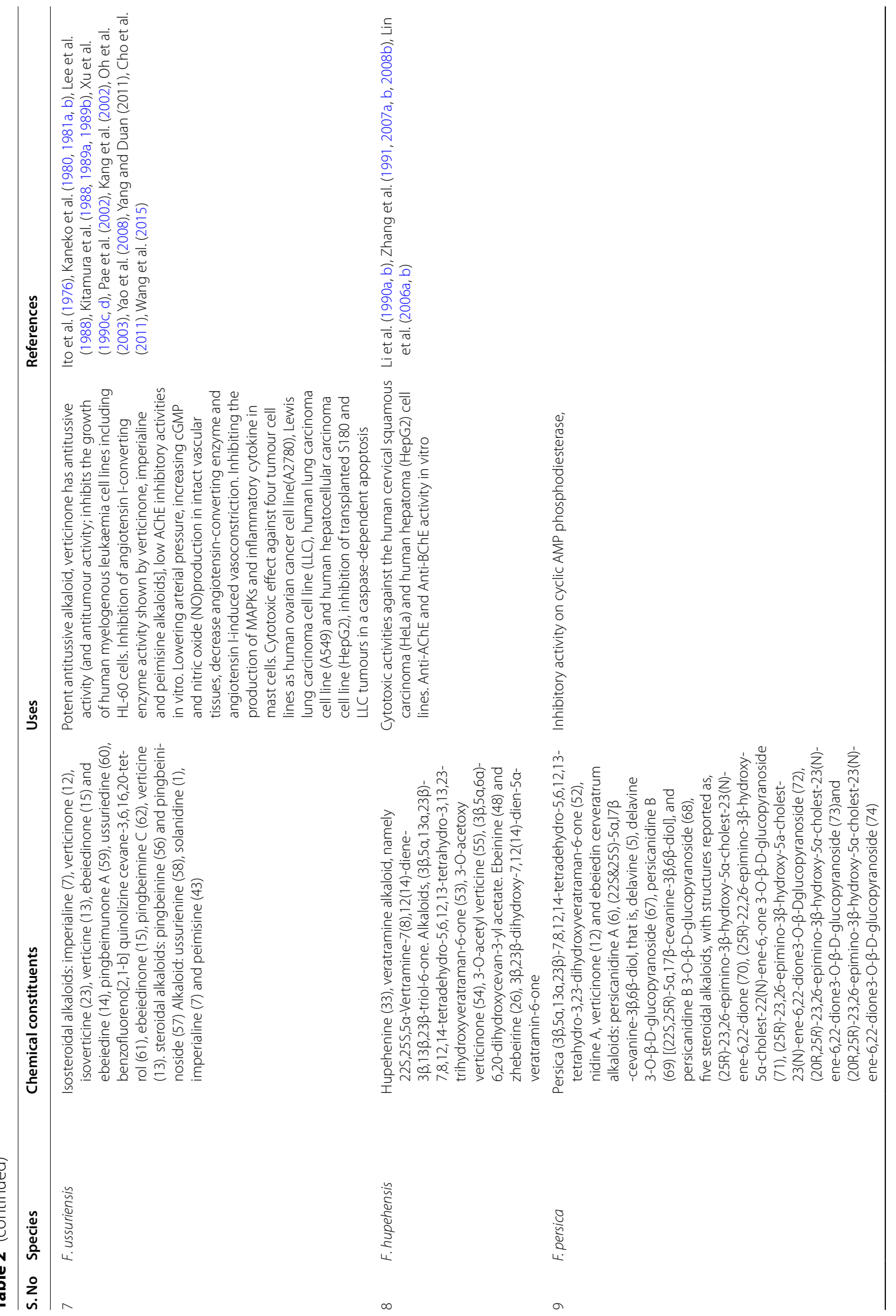




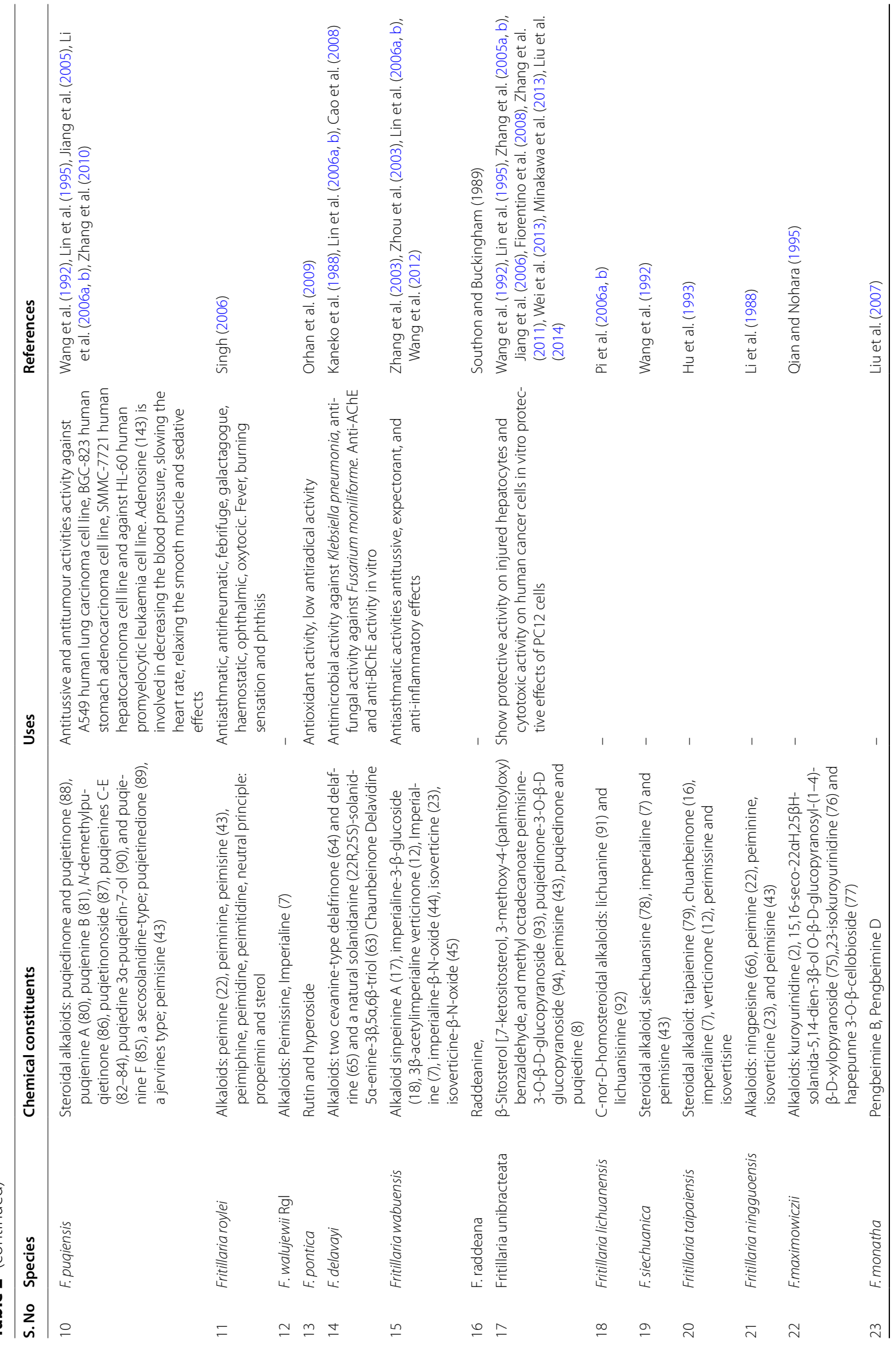




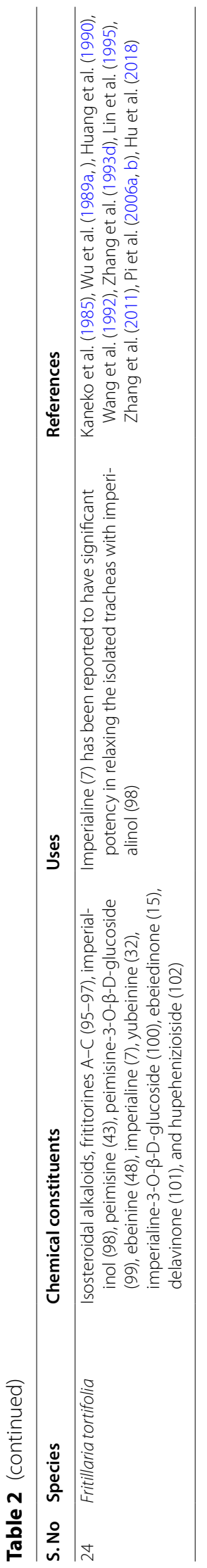



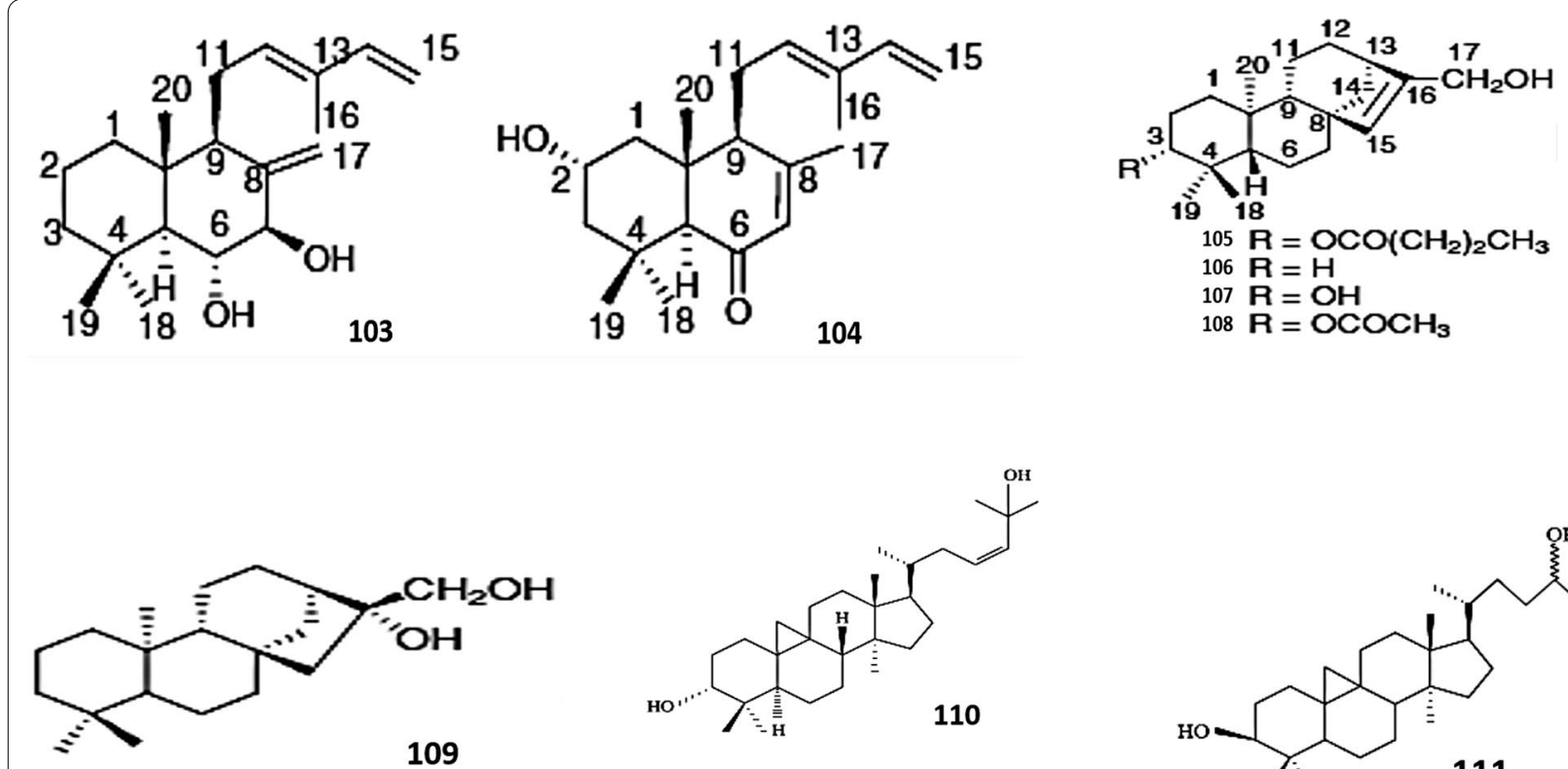

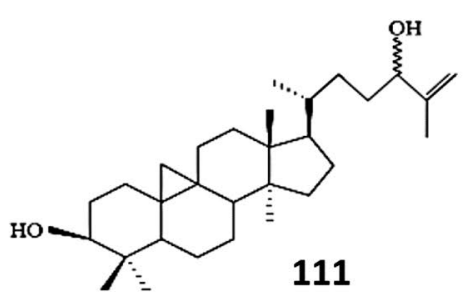

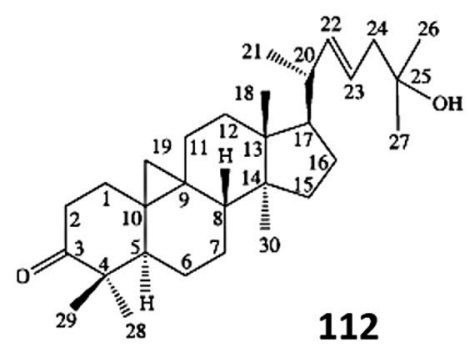

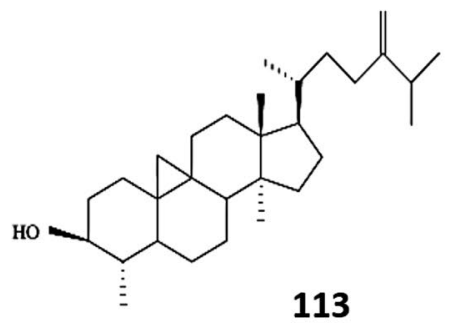
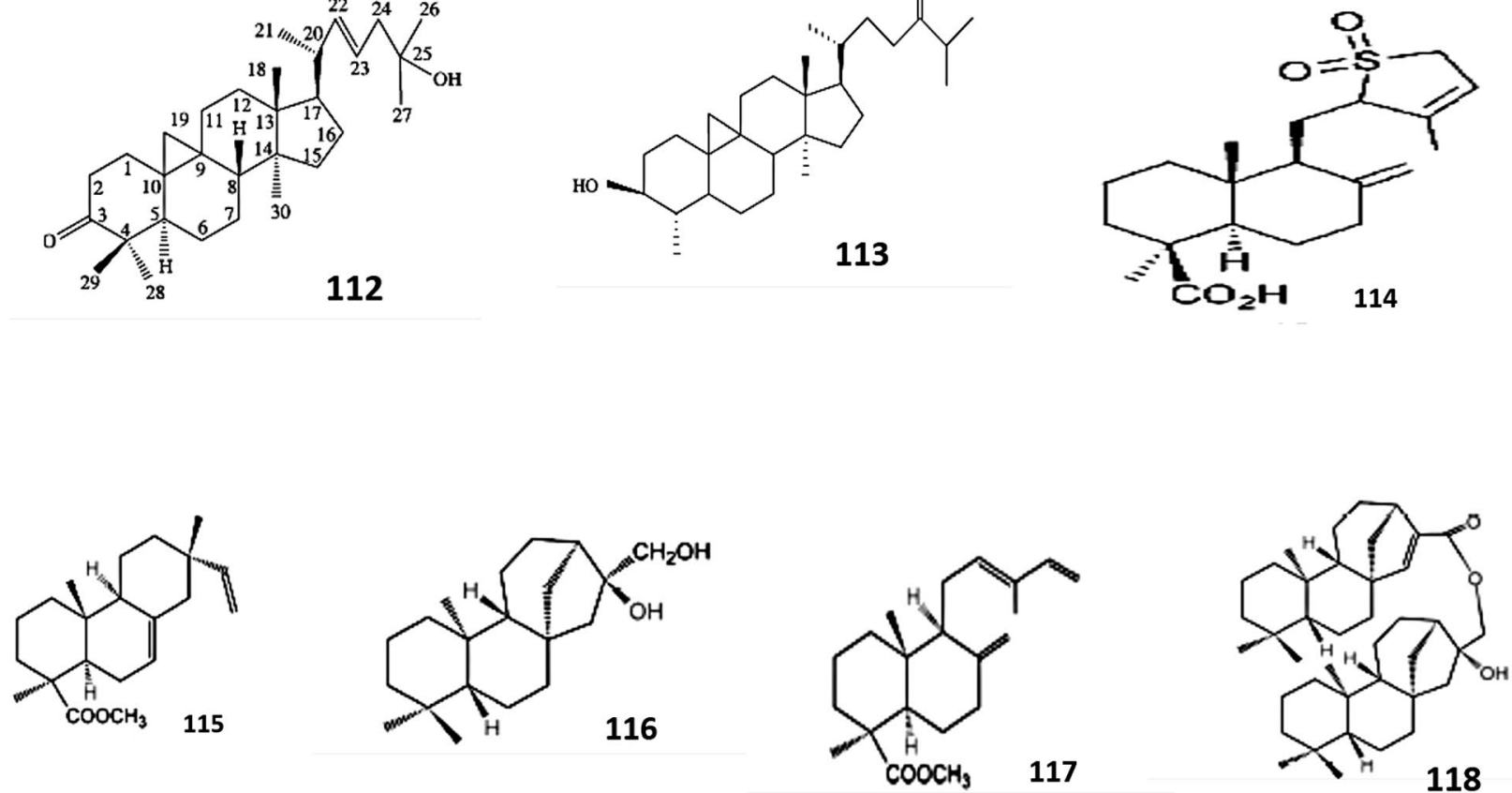

Fig. 2 Structures of various terpenoids of genus Fritillaria: 103. 6a,7 -dihydroxy-labda-8 (17),12(E),14-triene, 104. 6-oxo-2a-hydroxy-labda-7,12(E),

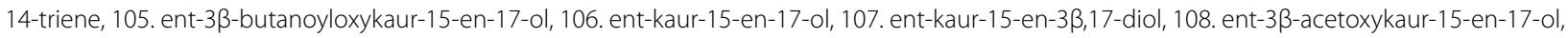
109. ent-kauran-16ß,17-diol, 110. triterpenoid (23z)- 9,19-cycloart-23-ene-3a,25-di-ol, 111. triterpenoid 9,19-cycloart-25-ene-3b,24j-diol, 112. triterpenoids 25-hydroxyl-9,19-cycloart-22-ene-3-one, 113. cycloeucalenol, 114. bicyclic diterpenoid labdane, 115. isopimaran-19-oic acid, methyl

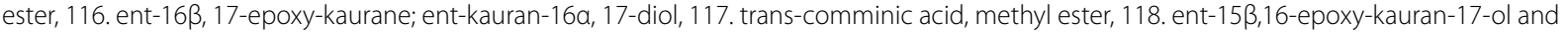
ent-16ß-hydroxy-kauran-17-yl ent-kaur-15-en-17-oate 
Table 3 Various terpenoids in genus Fritillaria and their uses

\begin{tabular}{|c|c|c|c|c|}
\hline S. No. & Species of Fritillaria & Chemical constituents & Uses & References \\
\hline 1 & Fritillaria imperialis & $\begin{array}{l}\text { Tricyclic diterpenes; isopimara- } \\
\text { 7,15-dien-19-oic acid, }\end{array}$ & $\begin{array}{l}\text { Prolyl endopeptidase inhibitory } \\
\text { activity }\end{array}$ & Atta-ur-Rahman et al. (2005) \\
\hline 2 & Fritillaria ebeiensis & 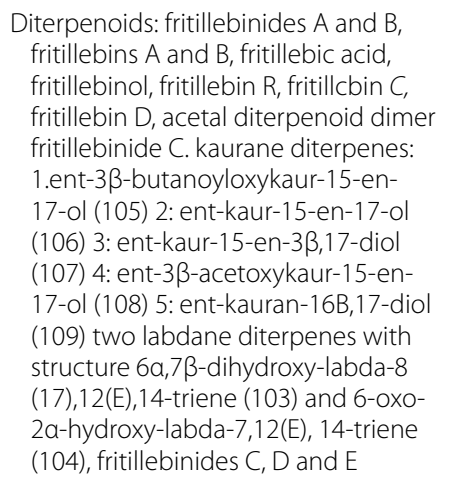 & $\begin{array}{l}\text { Antitussive and expectorant effects. } \\
\text { Neuroprotective activity against } \\
\text { 1-methyl-4-phenyl-pyridinium } \\
\left(\mathrm{MPP}^{+} \text {)-induced neuronal cell death }\right. \\
\text { in human dopaminergic neuroblas- } \\
\text { toma SH-SY5Y cells. Anti-AChE and } \\
\text { Anti-BChE activity in vitro. Strong } \\
\text { antitumour activity in inhibiting } \\
\text { the growth of the solid type of } \\
\text { hepatoma and Ehrlich ascites carci- } \\
\text { noma in mice }\end{array}$ & $\begin{array}{l}\text { Yu et al. (1985); Li et al. (1995a, b), } \\
\text { Bandara and Wimalasiri (1988), } \\
\text { Wu et al. (1999, 1995), Ruan et al. } \\
\text { (2002), Zhang et al. (2005a, b), Lin } \\
\text { et al. (2006a, b), Chen et al. (2008a, } \\
\text { b, 2011), Xu et al. (2011a, b), Zhang } \\
\text { et al. (2013) }\end{array}$ \\
\hline 3 & F. hupehensis & $\begin{array}{l}\text { Cycloartane-type triterpenoids; } \\
\text { 25-hydroxyl-9,19-cycloart- } \\
\text { 22-ene-3-one, (23Z)-9,19- } \\
\text { cycloart-23-ene-3a,25-diol,9,19- } \\
\text { cycloart-25-ene-3b,24j-diol, and } \\
\text { cycloeucalenol (113). Cycloartane } \\
\text { triterpenoids:triterpenoid (23Z)-9,19- } \\
\text { cycloart-23-ene-3a,25-diol (110) } \\
\text { and triterpenoid 9,19-cycloart-25- } \\
\text { ene-3b,24j-diol (111) triterpenoids } \\
\text { 25-hydroxyl-9,19-cycloart-22-ene-3- } \\
\text { one (112) }\end{array}$ & $\begin{array}{l}\text { Cytotoxic activities against the human } \\
\text { cervical squamous carcinoma (HeLa) } \\
\text { and human hepatoma (HepG2) } \\
\text { cell lines. Anti-AChE and Anti-BChE } \\
\text { activity invitro }\end{array}$ & $\begin{array}{l}\text { Goebel and Schrempf (1972), Wu } \\
\text { et al. (1999), Lin et al. (2006a, b), Pi } \\
\text { et al. (2007), Zhang et al. (2007a, b, } \\
\text { 2008b), Tong (2016) }\end{array}$ \\
\hline 4 & Fritillaria anhuiensis & BICYCLIC diterpenoid labdane (114) & - & Shou et al. (2009) \\
\hline 5 & F. thunbergii & 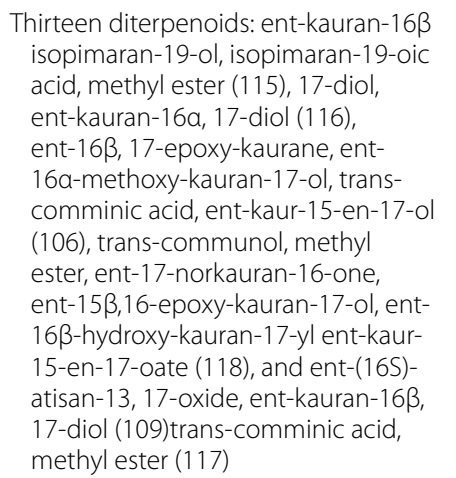 & - & Kitajima et al. (1982a, b) \\
\hline
\end{tabular}

more kaurane diterpenes (105-109) isolated from $F$. ebeiensis was reported as (Xu et al. 2011a):

Compound 105: ent-3 $\beta$-butanoyloxykaur-15-en-17-ol;

106: ent-kaur-15-en-17-ol (Liu et al. 2007);

107: ent-kaur-15-en-3 $\beta, 17$-diol (Bandara and Wimalasiri 1988; Liu et al. 2007)

108: ent-3 $\beta$-acetoxykaur-15-en-17-ol;

109: ent-kauran-16 $\beta, 17-$ diol.

In Fritillaria imperialis bulbs, a tricyclic diterpene isopimara-7,15-dien-19-oic acid (Atta-ur-Rahman, 2005) has been reported. From stems and leaves of $F$. hupehensis, chemical constituents have been analysed as cycloartane triterpenoids; cycloeucalenol (113) (Pi et al. 2009), triterpenoid (23z)- 9,19-cycloart-23-ene-3a,25di-ol (110) (Pi et al. 2007), triterpenoids 25-hydroxyl9,19-cycloart-22-ene-3-one (112) and triterpenoid 9,19-cycloart-25-ene-3b,24j-diol (111) (Yu et al. 1985) have been reported. Fritillaria anhuiensis has been examined to contain a bicyclic diterpenoid labdane (114) which contains a typical sulfonyl group (Shou et al. 2009). Thirteen diterpenoids including isopimaran-19-oic acid, methyl ester (115); isopimaran-19-ol; ent-kauran-16 $\beta, 17$ diol (109); ent-16 $\beta$, 17-epoxy-kaurane; ent-kauran-16 $\alpha$, 17-diol (116); trans-communol; ent-kaur-15-en-17-ol 

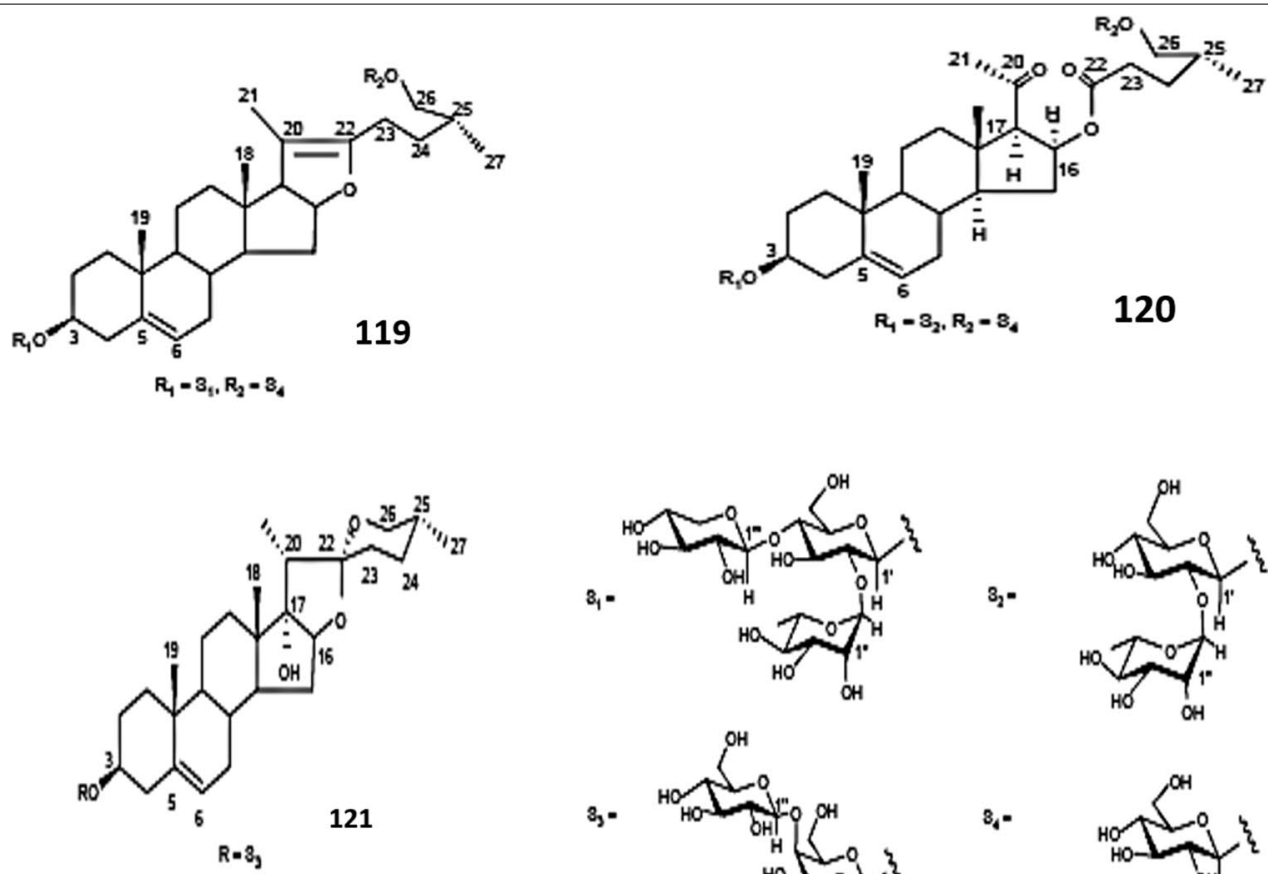

$3_{2}$.

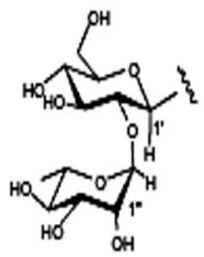

$3,-$
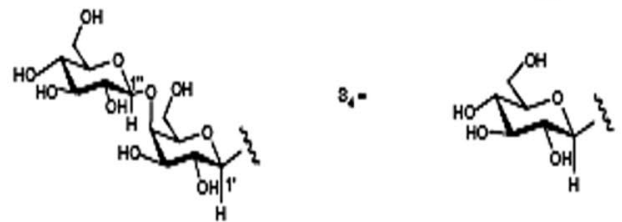

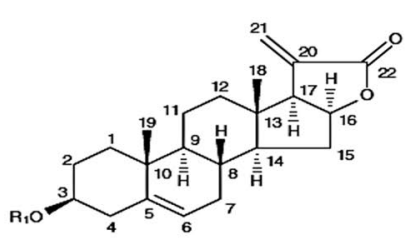

$122 \mathrm{R}_{1}=\mathrm{S}_{2}$

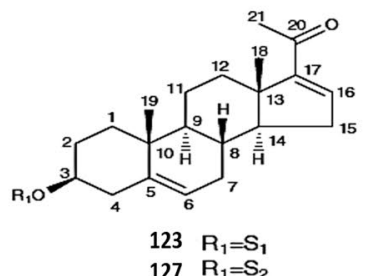

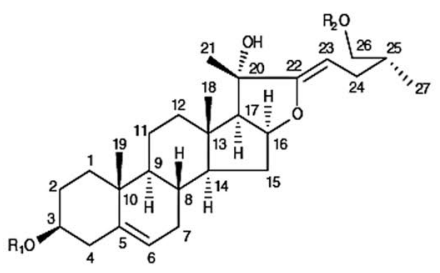

$128 R_{1}=S_{2}, R_{2}=S_{4}$ $124 R_{1}=S_{1}, R_{2}=S_{4}$

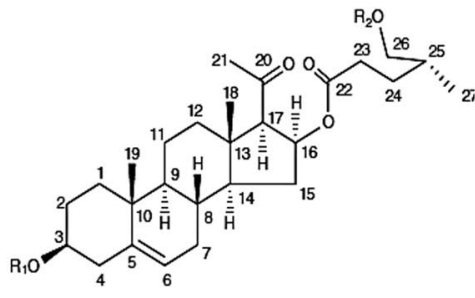

$125 \mathrm{R}_{1}=\mathrm{S}_{1}, \mathrm{R}_{2}=\mathrm{S}_{4}$

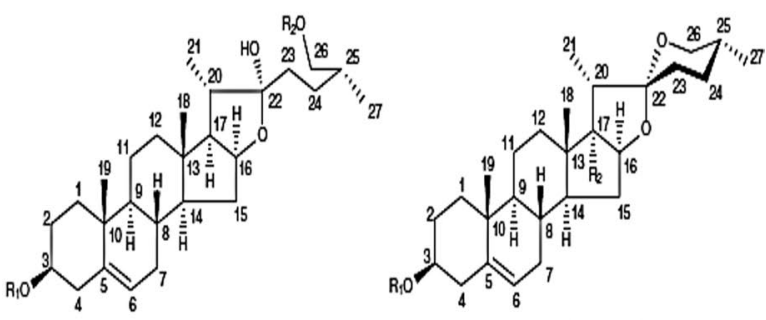

$126 R_{1}=S_{1}, R_{2}=S_{4}$

$129 R_{1}=S_{5,} R_{2}=S_{4}$

$130 R_{1}=S_{2}, R_{2}=S_{4}$

\section{$131 \mathrm{R}_{1}=\mathrm{S}_{2}, \mathrm{R}_{2}=\mathrm{OH}$ \\ $132 R_{1}=S_{2}, R_{2}=H$ \\ $133 R_{1}=S_{3}, R_{2}=H$}

Fig. 3 Structures of various other chemical compounds of genus Fritillaria: 119. pallidiflosides A, 120. pallidiflosides B, 121. pallidiflosides C, 122. pallidiflosides $D, 123$. pallidiflosides $E, 124$. pallidiflosides $G, 125$. pallidiflosides $H, 126$. pallidiflosides I, 127. spongipregnoloside $A, 128$. smilaxchinoside $C$, 129. timosaponin $H 1$, 130. protobioside, 131. polygonatoside B3, 132. polyphyllin $V, 133$. deltonin, 134. parispseudoside B,

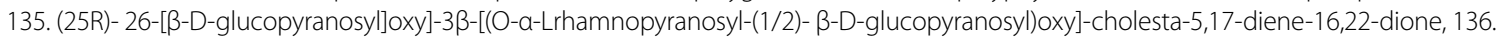

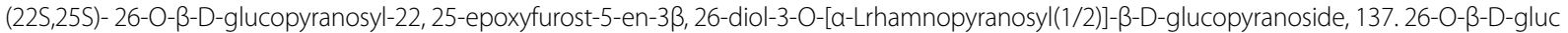

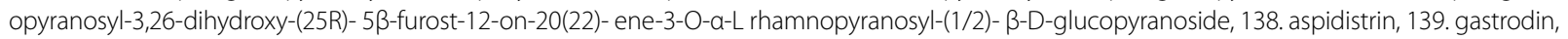
140. 4-( $\beta$-D-glucopyranosyloxy) benzoic acid, 141. icariside D2, 142. uridine, 143. adenosine, 144. uracil, 145. (S)- $\beta$-L-phenylalanine, 146. arginine, 147. lysine, 148. tryptophan, 149. tyrosine, 150. histidine, 151. isoleucine, 152. glycine, 153. leucine, 154. valine, 155. oxyproline, 156. alanine, 157. glutamate, 158. threonine, 159. proline, 160. methionine, 161. serine, 162. aspartate, 163. cysteine, 164. phenylalanine, 165. ornithine, 166. meristic acid C14:0, 167. pentadecanoic acid C15:0, 168. palmitic acid C16:0, 169. palmitoleic acid C16:1, 170. stearic acid C18:0, 171. oleic acid C18:1, 172. linoleic acid C18:2, 173. linolenic acid C18:3, 174. a -monopalmitin, 175. diosmetin, 176. murrayone, 177. 1-O- $\beta$-D-glucopyranosyl-(2S,3R,4E,8Z)-2-[(2-hydroxyoctadecanoyl) amido]-4, 8-octadecadiene-1,3-diol, 178. fritenolide A, 179. fritenolide $B, 180$. fritenolide C, 181. fritenolide $D, 182$. fritenolide $E$ 


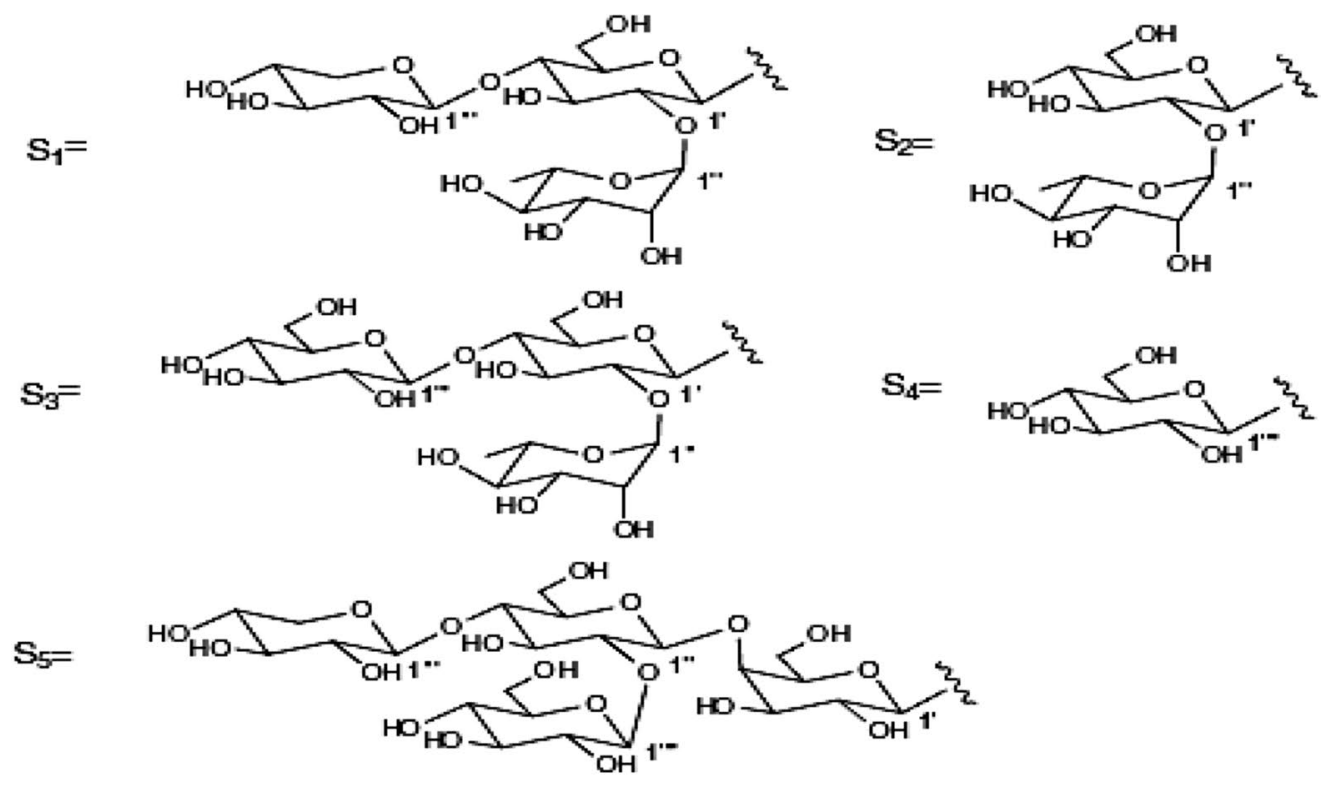

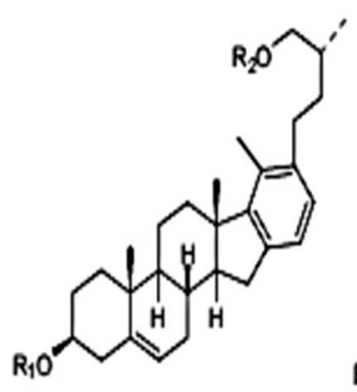

$134 \mathrm{R} 1=\mathrm{S} 1, \mathrm{R} 2=\mathrm{S} 2$

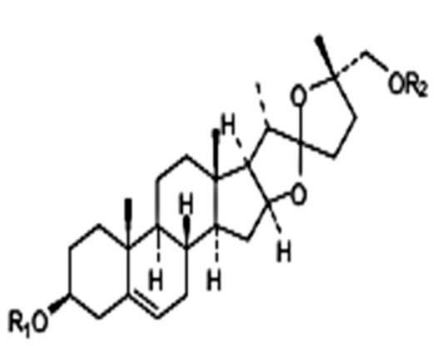

$136 \mathrm{R} 1=\mathrm{S} 1, \mathrm{R} 2=\mathrm{S} 2$

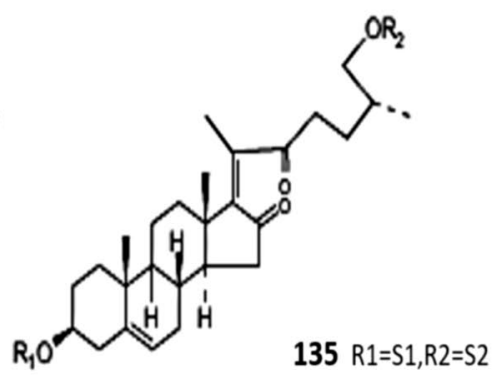

$135 \mathrm{R} 1=\mathrm{S} 1, \mathrm{R} 2=\mathrm{S} 2$

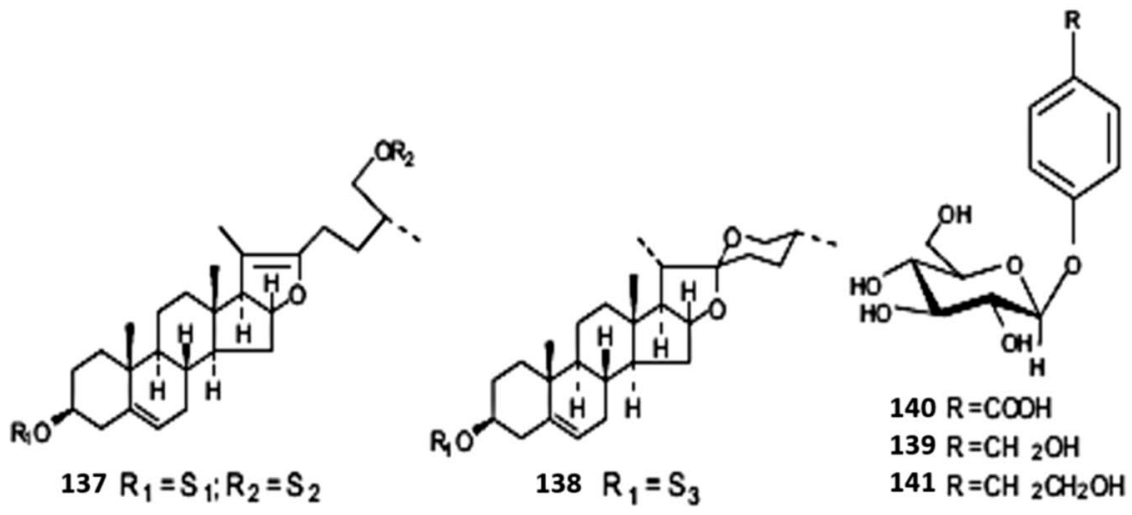

Fig. 3 continued

(106); trans-comminic acid, methyl ester (117); ent$15 \beta, 16$-epoxy-kauran-17-ol and ent-16 $\beta$-hydroxykauran-17-yl ent-kaur-15-en-17-oate (118) were isolated in two investigations conducted on F. thunbergii (Kitajima et al. 1982a, b, c). Table 3 shows different terpenoids in genus Fritillaria along with their uses.

\section{Other compounds}

The structures of these chemical constituents are given in Fig. 3. From the bulbs of Fritillaria imperialis, cevarin and cevacin (Chopra et al. 1956) were isolated; the volatile component of the floral bulbs of Fritillaria imperialis included 3-methyl-2-butene-1-thiol, 2-nitroethanol, 

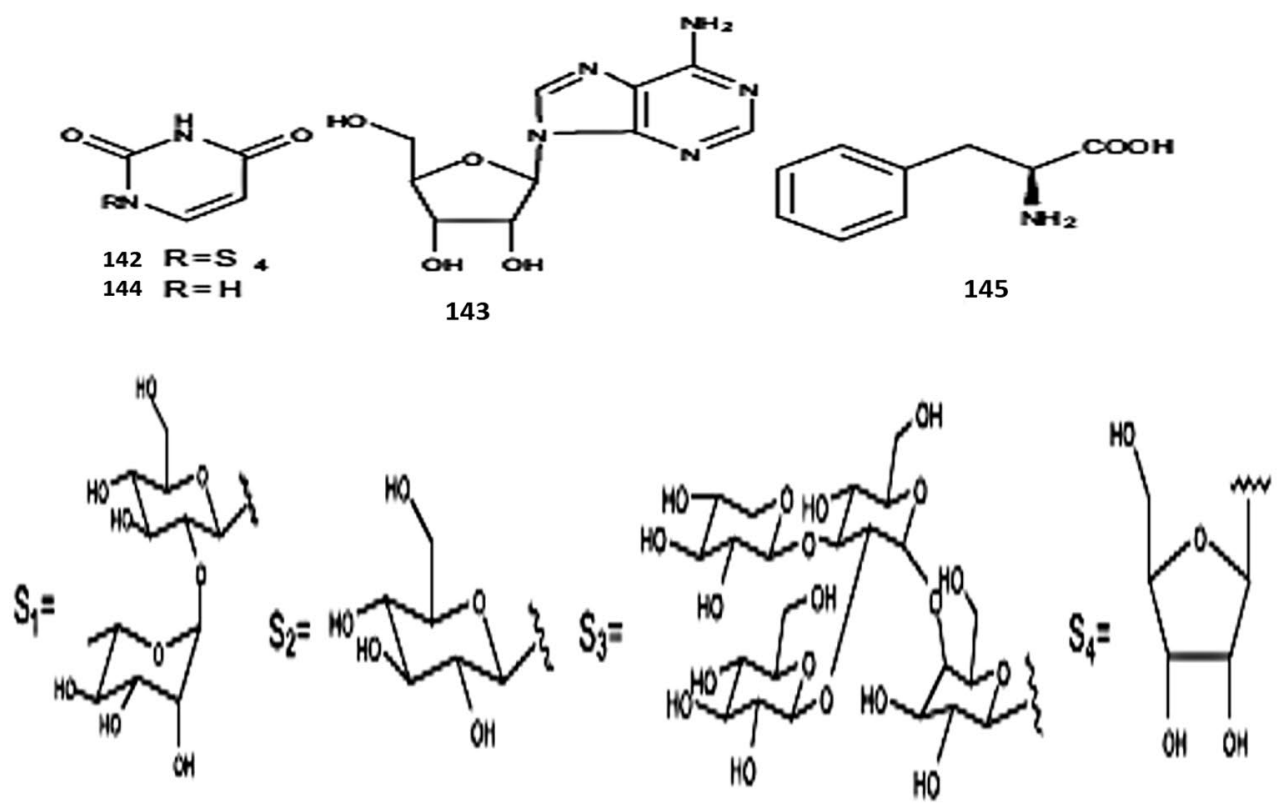<smiles>C=C(N)C(=N)CCCC(N)C(=O)O</smiles><smiles>N[C@@H](Cc1ccc(O)cc1)C(=O)O</smiles>

149

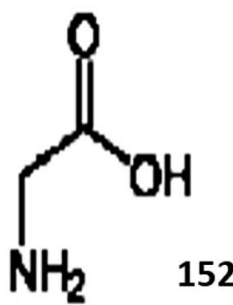<smiles>NCCCC[C@H](N)C(=O)O</smiles>

147<smiles>NC(Cc1c[nH]cn1)C(=O)O</smiles>

150<smiles>CC(C)CC(N)C(=O)O</smiles>

153<smiles>NC(Cc1c[nH]c2ccccc12)C(=O)O</smiles><smiles>CC[C@H](C)[C@H](N)C(=O)O</smiles>

151<smiles>CC(C)[C@H](N)C(=O)O</smiles>

Fig. 3 continued

3-methylpentanol, 2,3-butanediol, acidic acid, 3-pentene2-ol, 1-hexanol,3-hydroxy-2-butanone,cyclohexanone, 1,2-dimethyl benzene, dihydro-3-methyl $2(3 \mathrm{H})$ - furanone, benzaldehyde, hexadecane, octanoic acid, acetophenone, 4,6-trichlorophenol, decanal, tetradecane, nonanoic acid, pentadecane, 2-nonene-1-ol, 3,4-dimethyl-1,5-heptadiene, (Helsper et al. 2006). From Fritillaria verticillata bulbs, $\beta$-sitosterol-3-Oglucopyranoside a glucosylsterol has been isolated as main principal component (Kim et al. 2003). From Fritillaria pallidiflora, Schrenk bulbs steroidal saponins named pallidiflosides A (119), pallidiflosides C (121) and 
<smiles>O=C(O)C1CC(O)CN1</smiles><smiles>C[C@@H](O)[C@@H](N)C(=O)O</smiles><smiles>CC(C)(C)[C@@H](N)C(=O)O</smiles><smiles>C[C@H](N)C(=O)O</smiles><smiles>N[C](CCC(=O)O)C(=O)O</smiles>

157<smiles>CSCCC(N)C(=O)O</smiles><smiles>N[C@@H](Cc1ccccc1)C(=O)O</smiles><smiles>NCCCC(N)C(=O)O</smiles><smiles>CCCCCCCCCCCCCCCC(=O)O</smiles>

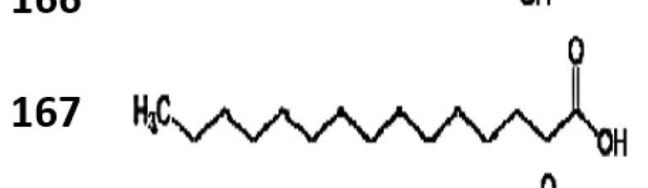<smiles>N[C@@H](CS)C(=O)O</smiles><smiles>CCCCCC/C=C\CCCCCCCC(=O)O</smiles>

171<smiles>CCCCCCCC/C=C\CCCCCCC(=O)O</smiles>

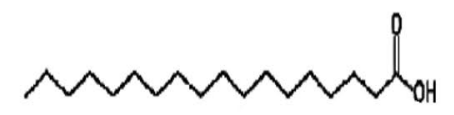




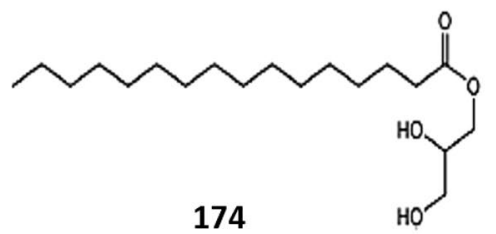

174

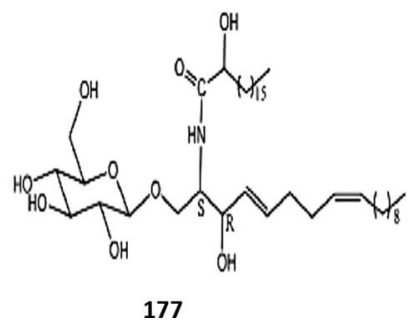

Fig. 3 continued<smiles>COc1ccc(-c2cc(=O)c3c(O)cc(O)cc3o2)cc1O</smiles>

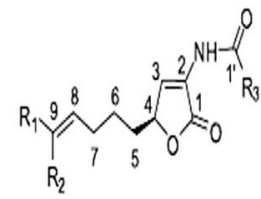

$178 \mathrm{R}_{1}=\left(\mathrm{CH}_{2}\right)_{8} \mathrm{CH}_{3}, \mathrm{R}_{2}=\mathrm{H}_{1} \mathrm{R}_{3}=\left(\mathrm{CH}_{2}\right)_{12} \mathrm{CH}_{3}$ $179 \mathrm{R}_{1}=\mathrm{H}_{1} \mathrm{R}_{2}=\left(\mathrm{CH}_{2}\right)_{8} \mathrm{CH}_{3} \mathrm{R}_{3}=\left(\mathrm{CH}_{2}\right)_{12} \mathrm{CH}_{3}$ $180 \mathrm{R}_{1}=\left(\mathrm{CH}_{2}\right)_{8} \mathrm{CH}_{3}, \mathrm{R}_{2}=\mathrm{H}_{1} \mathrm{R}_{3}=\left(\mathrm{CH}_{2}\right)_{10} \mathrm{CH}_{3}$<smiles>C=C(C)C(=O)Cc1c(OC)ccc2ccc(=O)oc12</smiles><smiles></smiles>

${ }_{181} R_{1}=\left(\mathrm{CH}_{2}\right)_{13} \mathrm{CH}_{3}, \mathrm{R}_{2}=\left(\mathrm{CH}_{2}\right)_{12} \mathrm{CH}_{3}$ ${ }_{182} \mathrm{R}_{1}=\left(\mathrm{CH}_{2}\right)_{13} \mathrm{CH}_{31} \mathrm{R}_{2}=\left(\mathrm{CH}_{2}\right) \mathrm{CH}_{3}$ pallidiflosides B (120) (Shen et al. 2011), pallidiflosides D (122), pallidiflosides E (123), pallidiflosides G (124), pallidiflosides H (125) and pallidiflosides I (126) (Shen et al. 2012a), spongipregnoloside A (127) (Yin et al. 2003), smilaxchinoside C (128) (Shao et al. 2007), timosaponin H1(129) (Meng et al. 1999), protobioside (130) (Geng et al. 2004), polygonatoside B3(131) (Ono et al. 2007), polyphyllin V (132) (Hou et al. 2006), deltonin (133) (Hayes et al. 2007), parispseudoside B (134) (Xiao et al. 2009); $(22 \mathrm{~S}, 25 \mathrm{~S})-26-\mathrm{O}-\beta-\mathrm{D}$-glucopyranosyl-22, 25-epoxyfurost5-en-3 $\beta$, 26-diol-3-O-[ $\alpha$-L-rhamnopyranosyl (1/2)]- $\beta$-Dglucopyranoside (136) (Mimaki and Sashida 1990a, b), (25R)- 26-[ $\beta$-D-glucopyranosyl $]$ oxy $]-3 \beta-[(\mathrm{O}-\alpha-\mathrm{L}-$ rhamnopyranosyl-(1/2)- $\quad \beta$-D-glucopyranosyl)oxy]cholesta-5,17-diene-16,22-dione (135) (Yokosuka and Mimaki 2008); aspidistrin (138) (Koketsu et al. 1996), and 26-O- $\beta$-D-glucopyranosyl-3,26-dihydroxy-(25R)$5 \beta$-furost-12-on-20 (22)- ene-3-O- $\alpha$ - L rhamnopyranosyl-(1/2)- $\beta$-D-glucopyranoside (137) (Dong et al. 2001) were isolated. Different compounds were identified including three phenolic glucosides; gastrodin (139) (Yang et al. 2007); icariside D2 (141) (Miyase 1989), and 4-( $\beta$-D-glucopyranosyloxy) benzoic acid (140) (Chen et al. 2008a, b), three nucleoside compounds adenosine (143) (Stueber and Grant 2002), uracil (144) (Ellis et al. 1973) and uridine (142) (Zhou et al. 2008), twenty one amino acids (S) - $\beta$-L-phenylalanine (145) (Tian et al. 2002), arginine (146), lysine (147), tryptophan (148), tyrosine (149), histidine e(150), isoleucine (151), glycine (152), leucine (153), valine (154), oxyproline (155), alanine
(156), glutamate (157), threonine (158), proline (159), methionine (160), serine (161), aspartate (162), cysteine (163), phenylalanine (164), ornithine (165), nine fatty acid: pentadecanoic acid C15:0(167), meristic acid C14:0 (166), palmitic acid C16:0(168), linoleic acid C18:2(172), oleic acid C18:1(171), palmitoleic acid C16:1(169), linolenic acid C18:3(173), stearic acid C18:0(170) (Utegenova et al. 2019), $\alpha$-monopalmitin(174). One coumarin: murrayone (176), one flavonoid: diosmetin (175), one sphingolipid: $1-\mathrm{O}-\beta-\mathrm{D}$-glucopyranosyl-(2S,3R,4E,8Z)-2[(2-hydroxyoctadecanoyl) amido]-4, 8-octadecadiene1,3-diol (177) (Jung et al. 1996) were likewise recognized in Fritillaria pallidiflora. Pharmacological and antimicrobial investigation on $F$. delavayi has detailed the presence of sterol, glycosides, volatile oil, saponins, reducing compounds, triterpenes, coumarins, quinones and flavonic glycosides (Maharjan et al. 2012). From Fritillaria unibracteata bulbs, five amino $\gamma$-butenolide compounds in particular fritenolide A (178), B (179), C (180), D (181), and $E$ (182) (Liu et al. 2014), and $\beta$-sitosterol (Wei et al. 2013), 7-ketositosterol (Zhang et al. 2005a, b), 3-methoxy4-(palmitoyloxy) benzaldehyde (Fiorentino et al. 2008) and methyl octadecanoate (Minakawa et al. 2013) were isolated. It was interesting to realize that the uncommon amide butenolide compounds were reported from thalassic lifeforms as six organic $\alpha$-amino butenolides acquired from the aquatic ascomycete Leptosphaeria orae-maris (White et al. 1989), bacterium Pseudoalteromonas sp. F-420 (Yoshikawa et al. 1997), sponge Anthosigmella aft. Raromicrosclera (Tsukamoto et al. 1995), and fungus 
Penicillium sp. No. 13 (Kimura et al. 2000) before their identification from terrestrial plants. In Fritillaria hupehensis Hsiao and K. C. Hsia non-basic components for example, fritillebin D, fritillebin C (Wu et al. 1999), thymidine (Goebel and Schrempf 1972), adenosine (143) and uridine (142) were reported. Phytochemical examination of $E$. thunbergii has reported volatile components: like some aromatic ketones and aldehydes, for example, benzeneacetaldehyde (Liang et al. 2011), octadecatrienoic acid methyl esters and twenty-one elements to be specific boron, sodium, magnesium, aluminium, phosphorus, sulfur, potassium, calcium, chromium, manganese, iron, cobalt, nickel, copper, zinc, molybdenum, lead, cadmium (Yao et al. 2008) arsenic, selenium and mercury (Wu and Zheng 1997; Wang et al. 2007; Liu et al. 2008; Cai et al. 2013; 2014; Zhou et al. 2014; Lou et al..2014) were identified. Alongside some different compounds including 29 components from essential oils as $\delta$-selinene; methyl ester; $\delta$-elemene; pentadecanoic acid (167); tetradecanoic acid; n-hexadecanoic acid; kaurene;heptadecanoic acid; 9,12-octadecadienoic acid (Z,Z)-, methyl ester; 9-tetradecenal, (Z)-; 9-hexadecenoic acid; linoleic acid, ethyl ester; 9,12-octadecadienoic acid, oleic acid (169); methyl ester, (E, E)-; (Cao et al.2012) L-(+)-ascorbic acid 2,6-dihexadecanoate; butylated hydroxytoluene; ethyl 9-hexadecenoate; hexadecanoic acid, ethyl ester; linoleic acid, ethyl ester; $2(1 \mathrm{H})$ - phenanthrenone, $3,4,4 \mathrm{a}, 4 \mathrm{~b}, 5,6,7$, kaur-16-ene; octadecanoic acid, ethyl ester; rost-4-En3-one,17-hydroxy-,(17.ß.); 3-methyleneandrostan-17-ol;

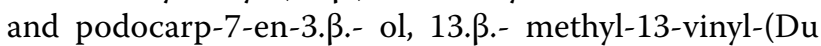
et al. 2018), six nucleosides including guanosine, uridine (142), adenosine (143), cytidine, thymidine, and inosine (Zhang et al. 2008a, 2011, 2016). Two sterols daucosterol and $\beta$-sitosterol (Zhang et al. 1993c), two carbohydrates sucrose and $\beta$-D-glucose4-1 $\beta$-D-galactose (Chen and Wang 2012), eighteen amino acids: leucine (153), glycine (152), methionine (160), histidine (150), tyrosine (149), threonine (158), isoleucine (151), alanine (156), tryptophan (148), lysine (147), cystine, aspartic acid, phenylalanine (164), valine (154), proline (159), glutamic acid, serine (161), and arginine (146) (Zhang et al. 2016), four nucleobases adenine, uracil (144), hypoxanthine and thymine (Zhang et al. 2008b, 2016), three fatty acid picropodophyllotoxin 2-monopalmitin and vernolic acid (Zhang et al. 1993e; Zhou et al. 2017), and three lignans zhebeiresinol, octahydrocurcumin and sauriol B (Jin et al. 1993; Zhou et al. 2017) were likewise recognized from Fritillaria thunbergii. Table 4 shows a list of different chemical constituents found in genus Fritillaria alongside their uses.

\section{Pharmacology}

The pharmacological investigations of the significant alkaloids of Fritillaria have shown that aside from hupehenine (33) and ebeienine (49) any remaining isosteroidal alkaloids of cevanine-type produce antitussive effects (Li et al. 1993; Chan et al.1999), despite the fact that the potency of the above alkaloids can change. In vitro investigation of bronchial and tracheal relaxation impacts of four principal bioactive isosteroidal alkaloids showing sequence of strength as imperialine (7), verticine (13), verticinone (12), ebeiedine (14) (Chan et al. 1999). As antitussive effect is concerned, imperialine (7) an isosteroidal alkaloid has been demonstrated to be the most powerful and least harmful compound among all alkaloids of genus Fritillaria (Chan et al. 1998). Phytochemicals confined from genus Fritillaria like verticine (13), imperialine- $\beta$ - $\mathrm{N}$-oxide (44), verticinone (12), imperialine (7), isoverticine (23), chuanbeinone (16) and isoverticine$\beta$-N-oxide (45) are known to show anti-inflammatory impact (Wang et al. 2011, 2012).

In species of Fritillaria like Fritillaria cirrhosa, Fritillaria pallidiflora and Fritillaria thunbergii the significant alkaloids specifically imperialine (7), verticinone (12), verticine (13), ebeiedine (14), and ebeiedinone (15) were investigated to have antitussive action ( $\mathrm{Li}$ et al. 1993) and are utilized in various cough status with respect to their potencies in conventional Chinese medication. Ebeinone extracted from Fritillaria imperialis bulbs shows anticholinergic effect in isolated tissue experiments, and it likewise blocks the inhibitory responses of acetylcholine totally (Sener 1994; Farooq et al. 1994), while alkaloids, impericine (3) and forticine (4), delavine (5), persicanidine A (6) and imperialine (7) are likewise known for their cholinesterase inhibitory activity (Akhtar et al. 2002). However, Fritillaria imperialis extracts were examined to show antibacterial, cytotoxic impacts and antiproliferative effects against three cancer cell lines HeLa (Human Cervix Carcinoma), HT29 (Human Colorectal Adenocarcinoma), C6 (Rat Brain Tumour Cells) and a non-malignant growth cell Vero (African Green Monkey Kidney). Additionally, antibacterial activity and cytoprotective action against Staphylococcus aureus and Escherichia coli (Aydın et al. 2018). From bulbs of F. persica, all the alkaloids were concentrated to show inhibitory action on cyclic AMP phosphodiesterase, except persicanidine B (69) which was inactive, whereas all other alkaloids were reported to show relatively high inhibitory activity (Ori et al. 1992a, 1992b, 1992c). In F. ussuriensis, verticinone (12) has been accounted for to repress the 


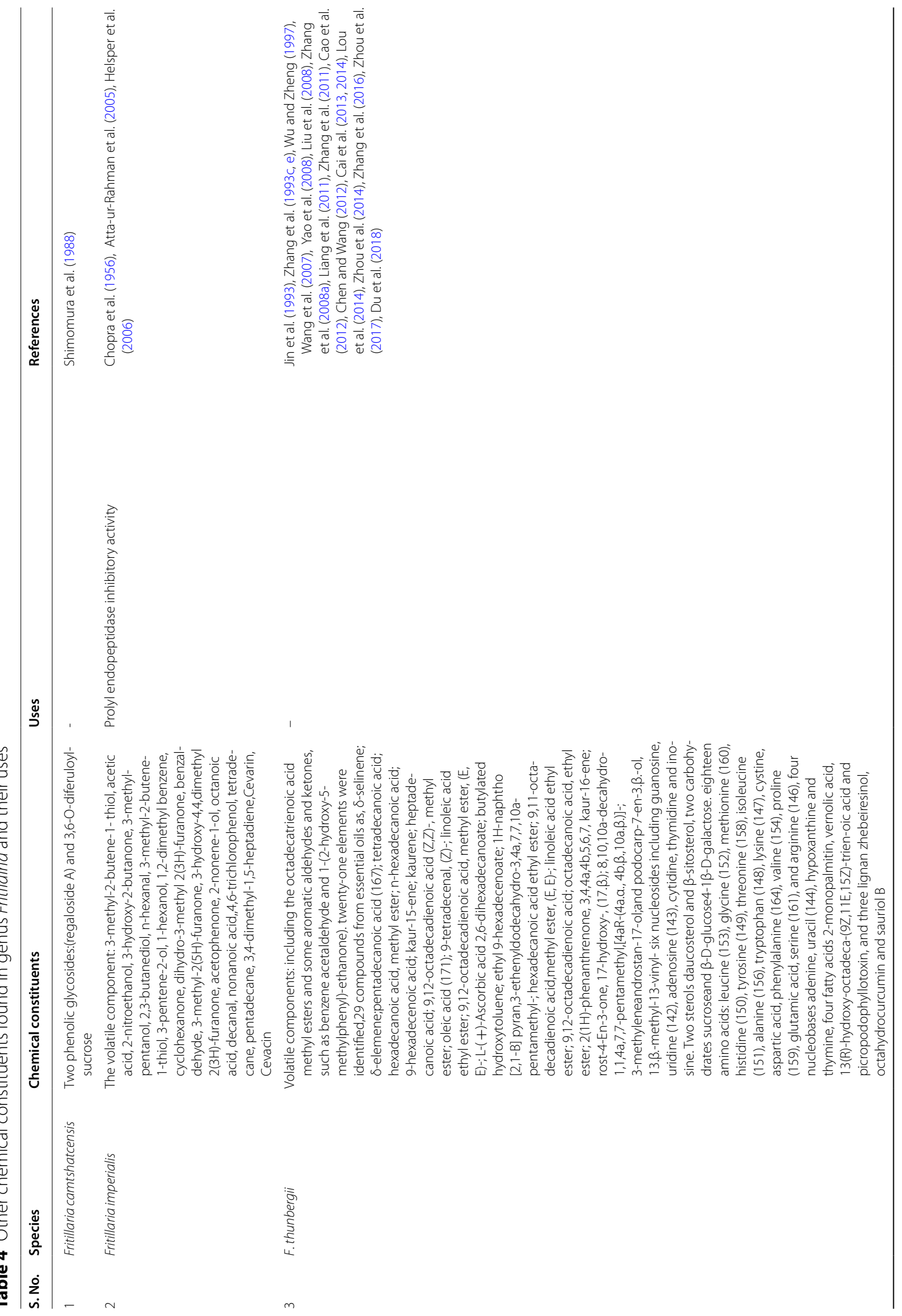




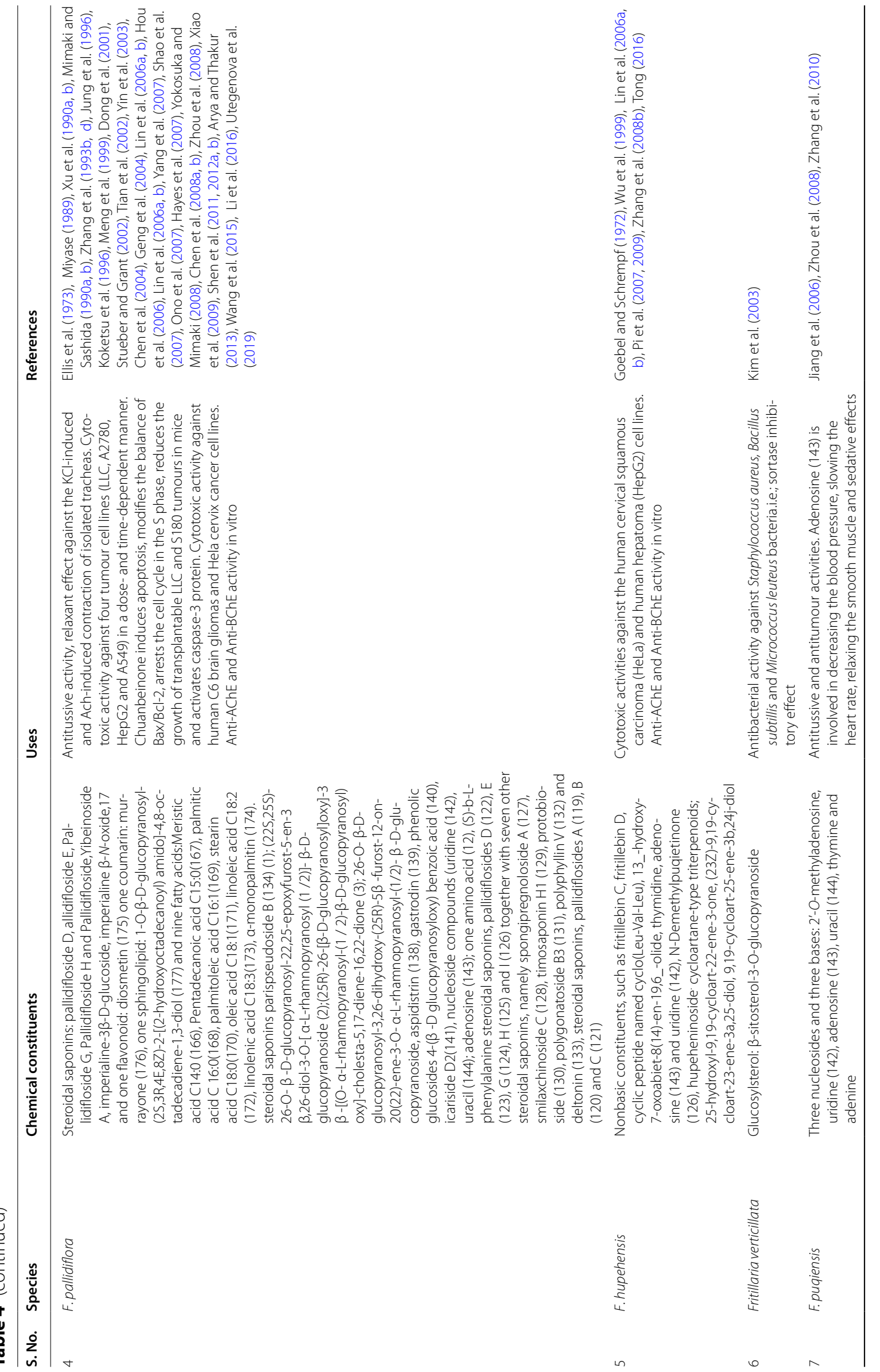




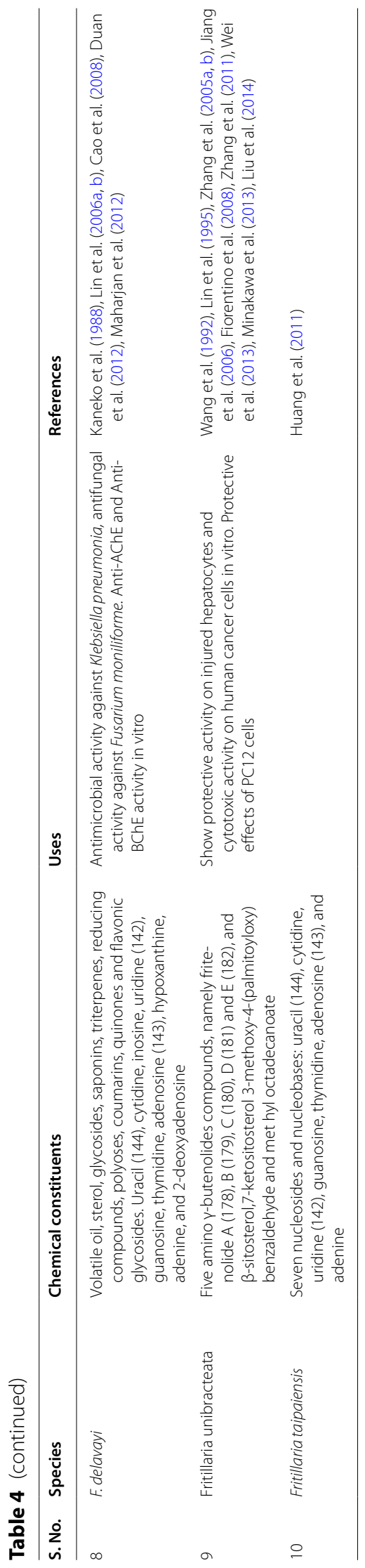


in vitro development of human myelogenous leukaemia cell lines involving HL-60 cells without inducing cell death. It on combination with ATRA (all-trans retinoic corrosive) induced the HL-60 cells differentiation, verticinone (12) decreases the impact of ATRA and furthermore shows more effectiveness (Pae et al. 2002). It is likewise known to have an antitussive action and antitumour action (Li et al. 1988, 1992, 1993). Different compounds as pingbeimunone A (59) (Yang and Duan 2012), ussuriedine (60) (Kitamura et al. 1989a), benzofluoreno[2,1-b] quinolizine cevane-3,6,16,20tetrol (61) (Kitamura et al. 1989b), ebeiedinone (15) (Lee et al. 1988), pingbeimine C (62) (Xu et al. 1990d, c), and verticine (13) (Kaneko et al. 1980) were identified and reported to show low AChE inhibitory actions in vitro in same species of genus Fritillaria (Yang and Duan 2012). Antitussive, expectorant and antiasthmatic effects of BFU (bulbus F. ussuriensis) are reported to be present because of its alkaloids content $(\mathrm{Qu}$ et al. 1990; Du 1996). Inhibition of angiotensin -I-changing enzyme action in a dose-dependent way is accounted for by in vitro investigation of verticinone (12), imperialine (7) and peimisine (43) alkaloids from BFU (Oh et al. 2003). Verticinone (12) is also studied in vitro to incite differentiation of Leukaemia HL-60 cells to granulocytes (Pae et al. 2002). In vivo investigations of BFU have detailed that its butanol and ethyl acetate extracts have action of bringing down the mean arterial pressure, rising cGMP and nitric oxide (NO) production in intact vascular tissues, and they decline angiotensinchanging enzyme and angiotensin I-influenced vasoconstriction (Kang et al. 2002). Other than, this ethanol extract is additionally known to repress the formation of MAPKs and inflammatory cytokine in mast cells (Cho et al. 2011). From Fritillaria ussuriensis bulbs, four steroidal alkaloids imperialine (7), peimisine (43), verticinone (12) and verticine (13) were extracted and identified, indicated surprising cytotoxic impacts and critical inhibitory impact is shown by peimisine (43) and verticinone (12) than the others. And all the four alkaloids show restriction of cell expansion in a timeand concentration-dependent manner (Lu et al.2004).

From F. cirrhosa and F. ussuriensis-peimisine (43), imperialine (7), verticine (13) and vericinone indicated critical cytotoxic impacts on A2780, HepG2, A549 and LLC cells (Wang et al. 2015). From Fritillaria ussuriensis and Fritillaria thunbergii known as "Ping-beimu" (Yang and Duan 2012) and "Zhe-beimu", respectively, isosteroidal alkaloids peimine (22) and peiminine were isolated as significant constituents of these species $(\mathrm{Xu}$ et al. 2016) demonstrating different pharmacological impacts (Li et al. 2006a) like antitumour (Lyu et al. 2015; Zheng et al. 2017), anti-inflammatory (Lee et al.
2015; Xu et al. 2016) antioxidant (Ruan et al. 2016) depressant and antitussive (Qian and Xu 1985) impacts. Peimine (22) are reported to repress the formation of pro-inflammatory cytokines, similar to IL-6, TNF- $\alpha$ and IL-8, and furthermore, in PMACI-induced HMC-1 cells, phosphorylation of MAPKs and NF-KB expression is also reduced (Park et al. 2017), ideally repressed the Kv1.3 ion channel and block the Nav1.7 ion channel (Xu et al. 2016). Peiminine is reported to protect dopaminergic neurons, inhibiting neuroinflammation and to treat Parkinson's infection (PD) and atopic dermatitis (AD) (Chen et al. 2018). From Fritillaria verticillata bulbs, $\beta$-sitosterol-3-O-glucopyranoside; a glucosylsterol has been extracted as a major functioning component, which shows sortase inhibitory effect. Sortase is a transpeptidase attaching surface protein in bacteria. The antibacterial action has been experimently demonstrated against Micrococcus luteus, Bacillus subtillis and Staphylococcus aureus bacteria (Kim et al. 2003). In gram-positive bacteria, sortase is associated with anchoring and secretion of cell wall protein (Pallen et al. 2001). From F. puqiensis steroidal alkaloids, puqiedinone and puqietinone (88) (Li et al. 1990a, ; Lin et al. 1995) were known for their antitussive and antitumour effects (Ji et al. 1993; Li et al. 1995a, b) while steroidal alkaloids of this species specifically puqienine B (81), puqietinonoside (87), $\mathrm{N}$-demethylpuqietinone (126) and puqienine A (80) were assessed to show various actions as follows:

1) antitussive action on cough induced by ammonia liquor in mice

2) activity on human lung carcinoma A549 cell line

3) activity on human stomach adenocarcinoma BGC823 cell line

4) activity on human hepatocarcinoma SMMC-7721 cell line

5) against human promyelocytic leukaemia HL-60 cell line (Jiang et al. 2005).

It was additionally revealed that Fritillaria puqiensis G. D. Yu et. G. Y. Chen, a local species of china Hubie Provience, acts as substitute for Beimu with its antitussive action (Ji et al. 1993; Li et al. 1995a, b). In addition, Fritillaria puqiensis crude alkaloids content and puqietinone (88) are accounted to block the development of three varieties of tumour (hepatoma, cervical carcinoma, Ehrlich ascites carcinoma) (Li et al. 1995a, b). The total alkaloids from Fritillaria hupehensis show antiasthmatic activity and have been reported by Xu et al. 2009. From Fritillaria hupehensis bulbs, cevan-based and six pentahexacyclic veratraman steroidal alkaloids were separated with the structure of compound 1, which was explained 
as 3-O-acetylverticine (55), compound 2 as $(3 \beta, 5 \alpha, 13 \alpha, 23 \beta)-\quad 7,8,12,14$-tetradehydro-5,6,12,13-tetrahydro-3,23-dihydroxyveratraman-6-one (52), compound 3 as $3-\mathrm{O}$-acetoxyverticinone (54), and structure of compound 4 was resolved as $(3 \beta, 5 \alpha, 13 \alpha, 23 \beta)$ 7,8,12,14-tetradehydro-5,6,12,13-tetrahydro-3,13,23-trihydroxyveratraman-6-one (53) (Zhang et al. 2008b), alongside two common alkaloids zhebeirine (27) and ebeinine (48) which were identified and evaluated to show cytostatic action against the HepG2 (human hepatoma) and HeLa (human cervical squamous carcinoma) cell lines of which compounds 2 and 4 demonstrated huge inhibitory impacts against the both kinds of tumour cells (Zhang et al. 2008b). From F. ebeiensis compounds like ent-kaur-15-en-3B,17-diol; ent-3B-butanoyloxykaur-15-en-17-ol, ent-kaur-15-en-17-ol (Bandara and Wimalasiri 1988; Liu et al. 2007), ent3B-acetoxykaur-15-en-17-ol and ent-kauran-16B,17-diol were isolated, from which compound 3 was accounted to show a solid neuroprotective impact at low concentration than other compounds, and these compounds did not show cytotoxcity action in the absence of MPP + . These diterpenoids were hence valuable in dealing with other neurological issues like Alzheimer's disease, Huntington's disease and Parkinson's disease (Xu et al. 2011a, b). Two labdane diterpenes with structure 6-oxo- $2 \alpha$-hydroxylabda-7,12(E), 14-triene (104), and $6 \alpha, 7 \beta$-dihydroxylabda-8 (17),12(E),14-triene (103) extracted from same species were reported to show neuroprotective impacts in human dopaminergic neuroblastoma SH-SY5Y neuronal cell death induced by MPP $+(\mathrm{Xu}$ et al. 2011a, b). From Fritillaria ebeiensis crude alkaloids, verticine (13), verticinone (12), ebeiedine (14) were reported in hepatoma in mice and Ehrlich ascites carcinoma showing strong growth inhibition, while strongest inhibition of tumours was shown by ebeiedine (14) (Li et al. 1995a, 1995b). The pharmacological investigations of the BFC (Bulbus F. cirrhosa) demonstrated alkaloids as principal constituents with expectorant, antiasthmatic and antitussive effects (Yan 2005; Chen 2008a, b). It has additionally been accounted that alkaloids like sinpeinine A (17), imperialine-3- $\beta$-glucoside (18), imperialine (7), verticinone (12) and $3 \beta$-acetylimperialine show strong antiasthmatic effect in vitro (Zhang et al. 2003; Zhou et al. 2003; Lin et al. 2006a, b). The pharmacological investigations of BFC (Bulbus Fritillaria cirrhosa)/BFW (Bulbus Fritillaria walujewii) showed crude alkaloids extracts with antitussive, antiasthmatic and expectorant actions (Yan 2005; Chen 2008a, b). Similarly, alkaloids like sinpeinine A (17), imperialine-3- $\beta$-glucoside (18), imperialine (7), verticinone (12) and $3 \beta$-acetylimperialine have been reported to have successful antiasthmatic actions (Zhang et al. 2003; Zhou et al. 2003; Lin et al. 2006a, b). Other alkaloids-imperialine (7), imperialine- $\beta$-N-oxide (44), isoverticine (23), and isoverticine- $\beta$ - $\mathrm{N}$-oxide (45)-were extracted from Fritillaria walujewii with their antitussive, anti-inflammatory and expectorant impacts (Wang et al. 2012) and in an investigation of alkaloids substances of Fritillaria cirrhosae bulbs, demonstrated striking antitumour action (Wang et al. 2014). Phytochemical investigation of alkaloids imperialine (7), verticinone (12) chuanbeinone (16) and verticine (13) isolated from BFC has resulted weaker expectorant impacts; however, more grounded antitussive and anti-inflammatory impacts of chuanbeinone (16), imperialine (7) than that of verticine (13), verticinone (12), which likewise showed that these alkaloids may act in a synergistic manner in the BFC (Wang et al. 2011). In Fritillaria thunbergii, isosteroidal alkaloids are significant phytochemicals revealed, of which peiminine and peimine (22) are two principle alkaloids constituents ( $\mathrm{Li}$ et al. 1992) and as indicated by the Chinese pharmacopeia these are considered as the marker components for the quality control in China likewise demonstrating anticancer impacts by restraining the development of tumour cells (Yang et al. 2005; Li et al. 2013; Liu et al. 2015; Tong 2016). The compounds show the order peimine $(22)>$ peiminine $>$ ebeiedine (14) $>$ puqietinone (88) in tracheobronchial relaxation in vitro (Chan 2000). From Fritillaria thunbergii ('Zhe Beimu' in Chinese), with antitussive and expectorant properties, the alkaloids-peimisine (43), peiminine and peimine (22) were isolated. They show the capacity of soothing cough, reducing sputum and furthermore indicating antioxidant action (Ruan et al. 2016). In recent investigations, it has been accounted that peiminine function as analgesic, antitussive and anti-inflammatory, helps to cure acute lung injury (Chan et al. 2000; Xu et al. 2011a, b; Guo et al. 2013), induces autophagic in cells, suppresses colorectal carcinoma cell expansion and fights cancer (Lyu et al. 2015). FTB additionally had other effects: prevent ulceration, antimuscarinic, neuroprotection, antithyroid, antidiarrheal, rheological properties and regulation of blood (Zhang et al. 1998a, 1998b; Jiang et al. 2005; Zhou et al. 2006; Suh et al. 2018; Zhang et al. 2018; Lin et al. 2010). In Fritillaria anhuiensis, a bicyclic diterpenoid labdane (114) has been reported to inhibit NO (nitric oxide) production notably (Popova et al. 2009). Phytochemical investigation has reported steroidal alkaloids from F. unibracteata bulbs, in particular puqiedinone-3-O- $\beta$-D-glucopyranoside (94), peimisine3-O- $\beta$-D-glucopyranoside (93) (Zhang et al. 2011), peimisine (43) (Wang et al. 1992), puqiedinone (Lin et al. 1995) and puqiedine (8) (Jiang et al. 2006) indicating protective effect of PC12 cells (Zhang et al. 2011). Moreover, 
five amino $\gamma$-butenolides compounds specifically fritenolide A (178), C (180), B (179), E (182) and D (181) (Liu et al. 2014), and $\beta$-sitosterol (Wei et al. 2013), 7-ketositosterol (Zhang et al.2005a, b), 3-methoxy4-(palmitoyloxy)benzaldehyde (Fiorentino et al. 2008) and methyl octadecanoate (Minakawa et al. 2013) were isolated and reported to show cytotoxic action on human cancer cells and defensive action on injured hepatocytes in vitro in same species (Liu et al. 2014). Antimicrobial and pharmacological examination on $F$. delavayi has reported the presence of triterpenes, volatile oil, sterols, saponins, polyoses, coumarins, glycosides, quinones and flavonic glycosides with antimicrobial activity against bacterial pathogen Klebsiella pneumoniae $(22 \mathrm{~mm})$, and most noteworthy inhibitory action was seen against a fungal pathogen, Fusarium moniliforme $(19 \mathrm{~mm})$. These antimicrobial actions recommended possible utilization of the plant in treatment of different diseases (Maharjan et al. 2012). Steroidal saponins are known to show antiinflammatory (Shao et al. 2007), antitumour (Furuya et al. 2001), antifungal (Zhang et al. 1993c), antithrombotic (Li et al. 2010), activities. Steroidal saponins, spongipregnoloside A (127) (Yin et al. 2003), pallidiflosides D (122), E (123), G (124), H (125) and I (126) (Shen et al. 2012a), smilaxchinoside C (128) (Shao et al. 2007), timosaponin H1(129) (Meng et al. 1999), protobioside (130) (Geng et al. 2004), polygonatoside B3(131) (Ono et al. 2007), polyphyllin V (132) (Hou et al. 2006) and deltonin (133) (Hayes et al. 2007) were extracted from the dry bulbs of $F$. pallidiflora some with cytotoxic action against Hela cervix cancer cell lines and human C6 brain gliomas, as steroidal saponin pallidiflosides D (122) and spirostanol saponins polygonatoside B3(131), polyphyllin V (132) and deltonin (133) showed cytotoxicity against Hela and C6 cells with compound deltonin (133) indicating the most strong cytotoxic action than other. It was likewise revealed that cytotoxic action revealed by these saponins is connected with the structures of sugar unit and aglycones (Shen et al. 2012a). Fritillaria pallidiflora found in Xinjiang territory, China, has been accounted to show antiasthmatic, antitussive and expectorant effects (Shen et al. 2011); the alkaloid substance of the plant was reported to show growth inhibition action on bacteria and strong antioxidant activity (Dang and Liu, 2013; Guo et al. 2013). Alkaloids were discovered to be primary constituents, isosteroidal alkaloids like imperialine- $\beta-\mathrm{N}$ oxide (44), chuanbeinone (16), isoverticine- $\beta$ - $\mathrm{N}$-oxide (45) and isoverticine (23) were reported to show significant cytotoxic action against four types of tumour cell lines (A2780, LLC, A549, and HepG2) in concentration and time-dependent manner and chuanbeinone (16) was discovered more effective than other three compounds. Chuanbeinone (16) was additionally reported to alter the equilibrium of $\mathrm{Bax} / \mathrm{Bcl}-2$, induce apoptosis, reduce the development of transplantable LLC, S180 tumours in mice, seize the cell cycle in the $S$ phase and activates caspase-3 protein (Wang et al. 2015). From Fritillaria pallidiflora and bulbs of Fritillaria ebeiensis, steroidal saponins counting pallidifloside D (122), E (123), G (124), $\mathrm{H}$ (125), I (126)) and kaurane diterpenes were isolated, respectively, and the previous demonstrated cytotoxic action against Hela and C6 cervix cancer cell lines (Shen et al. 2012a) and the later show neuroprotective impacts in human dopaminergic neuroblastoma SH-SY5Y neuronal cell death induced by MPP (Xu et al.2011a, b). Fritillaria pallidiflora (Yi Bei-Mu) utilized in conventional Chinese medication as antitussive, expectorant and antiasthmatic agents (Li et al. 1993; Zhou et al. 2003). Phytochemical investigation revealed fifteen isosteroidal alkaloids including ten cevane-type ones (Liu et al. 1984; $\mathrm{Xu}$ et al. 1990a; 1993; Xu et al. 2014), four jervine-type ones (Xu et al. 1990a, 1992) and a veratramine-type alkaloid (Shen et al. 2012b; Hao et al. 2013); cevane-type isosteroidal alkaloids in particular imperialine (7), verticine (13), ebeiedine (14) and verticinone (12) were isolated to act as the muscarinic M2-specific antagonist showing relaxant impact on smooth muscle (Eglen et al. 1992; Lin et al. 2006a, b; Kitazawa et al. 2007; Wang et al. 2012). Isosteroidal alkaloids from $F$. pallidiflora, yibeinones A-D (39-42) (Li et al. 2016), imperialine (7), imperialine-3ßD-glucoside (35), imperialine $\beta$-N-oxide (44) (Chen et al. 2004) and dongbeinine (19) (Zhang et al. 1993b) were isolated from which a few compounds indicated relaxation impact on tracheal preparation-compound 7 shows relaxant impact against the $\mathrm{KCl}$-induced compression of isolated tracheas, while as compounds $40,41,42$, and 7 demonstrated relaxation power on the Ach-induced tracheal preparation (Li et al. 2016). From Fritillaria tortifolia X. Z. Duan et X. J. Zheng, compound frititorines C (97) is a jervine-type alkaloid and imperialinol (98) is another natural cevanine-type alkaloid. Imperialine (7) is known to possess remarkable effect in relaxing the isolated tracheas (Li et al. 2016) with imperialinol (98).

A brief account of morphology of some of the species is given below:

a. Fritillaria imperialis, commonly called crown imperial, is an impressive plant, grows about $1 \mathrm{~m}(3 \mathrm{ft})$ in height. Each bulb produces a thick, stout, upright, ramrod-straight flowering stem which bears lanceshaped, glossy leaves with wavy margins appear in whorls around the lower $1 / 2$ of the stem. It bears a prominent whorl of 3-5 drooping or downward facing, bell-shaped orange or red flowers at the top of the stem, topped by a 'crown' of small pineapple-like tuft of leaf-like bracts. While the wild form is usually 


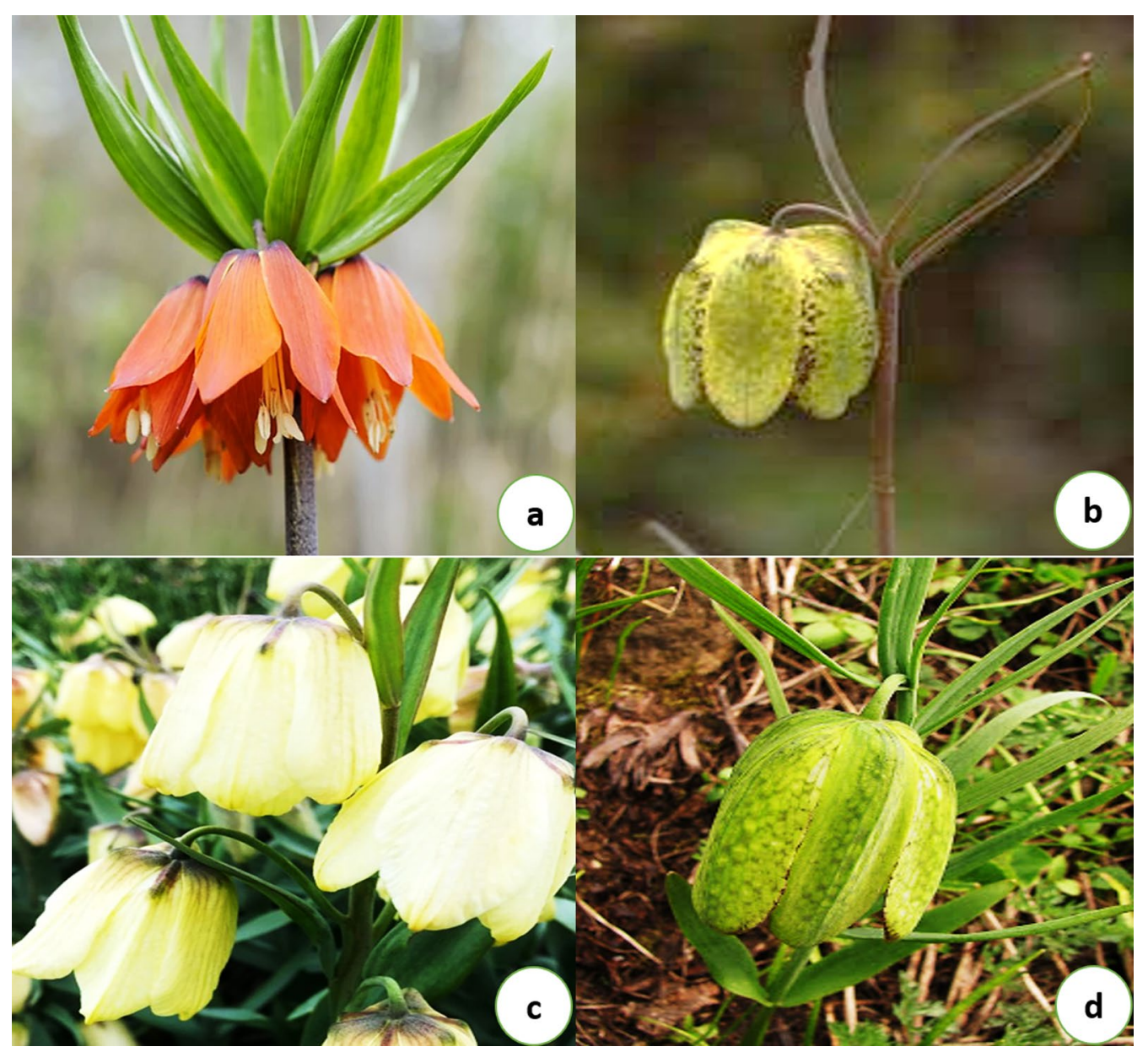

Fig. 4 a Fritillaria imperialis, b Fritillaria cirrhosa, c Fritillaria pallidiflora, d Fritillaria roylei

orange-red, various colours are found in cultivation, ranging from nearly a true scarlet through oranges to yellow. All parts of the plant have a skunky order (Fig. 4a).

b. Fritillaria cirrhosa is a perennial herb producing bulbs up to $20 \mathrm{~mm}$ ( $0.8 \mathrm{in})$ in diameter. Stem is up to $60 \mathrm{~cm}$ (24 in) tall with oppositely arranged leaves 7-11 in number, sometimes $3-4$ whorled and alternate. Leaves are linear to linear lance-shaped with the tip often curved or coiled. The plant bears nodded, bell-shaped, one flower at the top, sometimes flowers are in groups of one or three subtended by three leaflike bracts. Flower stalks (pedicel) shorter than tepals. Tepals are yellowish-green to brownish-purple, usually with a chequered pattern in dull purple. It is in flower from April to May. The species is hermaphrodite (has both male and female organs). Stamens are $2-3 \mathrm{~cm}$ long, style is three lobed, capsule is broadly oblong, $25-35 \mathrm{~mm}$ long, winged. Seeds are many and are pollinated by insects (Fig. 4b). c. F. pallidiflora is a perennial reaching up to $38 \mathrm{~cm}$ (15 in) in height, stems are thick having broadly lance-shaped bluish leaves scattered up and down the slender stem. Fritillaria pallidiflora bears 1-5 nodding greenish-yellow bell-shaped flowers $3-4 \mathrm{~cm}$ in length flowers are usually faintly checkered brownish red inside, pale yellow, nodding bell-shaped flowers. It is in flower from May to June. The species is hermaphrodite (has both male and female organs) and is pollinated by insect (Fig. 4c).

d. Fritillaria roylei is a herbaceous plant, $0.5-2 \mathrm{ft}$ tall, flowers are yellowish-green to brownish-purple and usually with a chequered pattern in dull purple. Flowers are broadly bell-shaped, hanging looking down, borne singly on the stems, but sometimes in groups of 2-4. Petals are narrow-ovate, $4-5 \mathrm{~cm}$ long. Leaves are linear-lancelike, often long-pointed, 5-10 cm, arranged oppositely or in whorls of 2-6 on the stem. It is in flower from April to May. The flowers are her- 
maphrodite (have both male and female organs) and are pollinated by insects (Fig. $4 \mathrm{~d}$ ).

\section{Conclusion}

The review highlighted the traditional uses, phytochemistry and pharmacology of different species of genus Fritillaria. Numerous Fritillaria species have been utilized in traditional Chinese medication for more than 2000 years due to their activities of reducing heat, alleviating cough, moistening the lung etc., for the treatment of bronchitis, a low sputum dry cough, asthma, tumours, struma, hemoptysis and insufficiency of milk and so on. Expanding interest in the field of plant as therapeutic assets has prompted significant discoveries of numerous essential compounds like alkaloids, terpenoids, saponins, nucleosides, flavonoids, glycosides, volatile components, nucleosides, amino acids, nucleobases, fatty acids and so on in different Fritillaria species including Fritillaria anhuiensis, Fritillaria cirrhosa, Fritillaria ebeiensis, Fritillaria hupehensis, Fritillaria imperialis, Fritillaria pallidiflora, Fritillaria puqiensis and Fritillaria thunbergii. However, around $80 \%$ of the Fritillaria species are yet to be investigated through phytochemical examinations which confine therapeutic and remedial utilization of products of Fritillaria. Fritillaria is utilized worldwide as medication and food traditionally and therapeutically because of its significant effects like anticholinergic action, cholinesterase inhibiting activity, antitussive and expectorant effects as well as neuroprotective action, anti-AChE and anti-BChE action, cytotoxic activity against tumour cell, and defensive action on injured hepatocytes, etc. Genus Fritillaria is utilized for the treatment of dyspepsia, chest injury, tuberculosis, gout, dysuria, sinus, boils, stomatitis, malaria, insanity, anaemia, immunity promoter, remedy for child emaciation, likewise for fever, burning sensation, phthisis and broncho-asthma, heart diseases, dysfunction of breathing and nervous system, etc. It is critical to study more species of genus Fritillaria for finding various compounds with important clinical efficiency and for its liveable utilization as medicinal resources. More significantly, research of Fritillaria ought not be confined to the pharmaceutical studies only but the other detailed studies like biochemistry, genetics, epigenetics, cytology and other fields to explore this important genus completely, which will assume an amazing part in future investigations of Fritillaria.
Acknowledgements

Not applicable.

Authors' contributions

IR contributed mostly in writing of the manuscript and collection of information. UY framed the idea and revised the whole manuscript after the writing. All the authors have read and approved the manuscript.

\section{Funding}

Not applicable.

Availability of data and materials

Not applicable.

\section{Declarations}

Ethics approval and consent to participate

Not applicable.

Consent for publication

Not applicable.

\section{Competing interests}

Not applicable.

\section{Author details}

${ }^{1}$ Department of Botany, S. P. College Campus, Cluster University, Srinagar, J\&K 190008, India. ${ }^{2}$ Department of Botany, Sri Pratap College, M. A. Road, Srinagar, J\&K 190001, India.

Received: 13 February 2021 Accepted: 14 June 2021

Published online: 12 July 2021

\section{References}

Abbaszadeh S, Teimouri H, Farzan B (2019) An ethanobotanical study of medicinal plants with antianxiety and antidepressant effects in shahrekord. Ept J Veterinary Sci 50(1):81-87

Akhtar MN, Choudhary MI, Tsuda Y, Sener B, Khalid A, Parvez M (2002) New steroidal alkaloids from Fritillaria imperialis and their cholinesterase inhibiting activities. Chem Pharm Bull 50(8):1013-1016

Akhtar MN, Choudhary MI, Sener B, Erdogan I, Tsuda Y (2003) New class of steroidal alkaloids from Fritillaria imperialis. Phytochemistry 63(1):115-122

Arya V, Thakur R (2013) Plant saponins-a recent update. Asian J Res Chem 6(9):871-876

Atta-Ur-Rahman, Nadeem Akhtar M, Iqbal Choudhary M, Tsuda Y, Yasin A, Sener B, Parvez M (2005) New diterpene isopimara-7, 15-dien-19-oic acid and its prolyl endopeptidase inhibitory activity. Natural Prod Res 19(1):13-22

Aydın A, Dede E, Elmastaş M, Tekin S (2018) Biological property of fritillaria imperialis L. extract. J Turk Chem Soc Sect A Chem 5(3):1043-1050

Bailey LH (1966) Manual of cultivated plants. MacMillan Company, New York, pp 218-219

Balkrishna A (2012) Secrets of astavarga plants. Sixth Edition. Haridwar (India): Divya Prakashan, 21-29

Bandara BR, Wimalasiri WR (1988) Diterpene alcohols from Croton lacciferus. Phytochemistry 27(1):225-226

Bensky D, Gamble A (1993) Herbs that expel parasites. Chinese Herbal Medicine Materia Medica. Eastland Press Inc., Seattle, pp 441-444

Bensky D, Clavey S, Stoger E (2004) Materia medica. Chinese Herbal Medicine, pp 3-6

Cai W, Xiong Y, Sheng Z, Xia M, Cui M (2013) Determination of eighteen trace elements in Fritillaria from different places by ICP-OES. Chin J Mod Appl Pharm 30:277-280

Cai X, Zhang Y, Du W, Sheng Z, Yin Z, Fang L, Cai B, Ge W (2014) Determination of heavy metal and trace elements in fresh-cut Bulbus Fritillaria thunbergii slices by ICP-OES. Chin Arch Tradit Chin Med 32:2357-2359 
Cao XW, Chen SB, Li J, Xiao PG, Chen SL (2008) Steroidal alkaloids from the bulbs of Fritillaria delavayi Franch. (Liliaceae). Biochem Syst Ecol 8(36), pp 665-668

Cao Y, Zhu X, Tan L (2012) Analysis of chemical composition by GC/MS and antimicrobial activities of essentialoil from Fritillaria thunbergii Miq. J. Zhejiang Sci.-Tech Univ 29:129-132

Chan SW (2000) Pharmacological and chemical investigations into bulbus fritillariae. Ph.D. Thesis, Chinese University of Hong Kong, Hong Kong, China

Chan SW, Kwan YW, Lin G, Ho YP, Li P (1998) The effects of Fritillaria alkaloids on rat isolated trachea and bronchi. Pharm Sci 1:365-369

Chan SW, Li SL, Lin G, Kwan YW, Li P (1999) Proceedings of the Hong Kong Pharmacology Society 5th Scientific Meeting, Hong Kong, p 21

Chan SW, Li SL, Lin G, Li P (2000) Pharmacokinetic study and determination of imperialine, the major bioactive component in antitussive Fritillaria cirrhosa, in rat by high-performance liquid chromatography coupled with evaporative light-scattering detector. Anal Biochem 285:172-175

Chen XQ (1980) Flora of China, 14th edn. Chinese Scientific. Press, Beijing

Chen MH (2008) Studies on Fritillaria cirrhosa D. Don and its effects of antitussive and antiasthma. Qinghai Normal University

Chen JD, Qiu Y, Yang ZW, Lin P, Lin YM (2008) Dimeric diterpenes from the roots of the mangrove plant Ceriops tagal. Helvetica Chim Acta 91(12):2292-2298

Cheng X, Xiao X, Zhang N, Ma S (2008) Determination the contents of peimine and peiminine in Thumberg Fritillary Bulb by HPLC-MS. China Pharm 11:770-772

Chen Y, Wang S (2012) $\beta$-D-glu 4-1 $\beta$-D-gal and sucrose determination of different Fritillarias by HPLC-ELSD.Lishizhen Med. Mater Med Res 23:1605-1606

Chen Q, Zhu LH, Xu YF, Fan JZ (2004) A new steroidal alkaloid from the bulbus of Fritillaria wabuensia. Acta pharmaceut Sinica 39(5):348-350

Chen X, Zhang J, Liu JH, Yu BY (2008b) Biotransformation of p-, m-, and o-hydroxybenzoic acids by Panax ginseng hairy root cultures. J Mol Catal B Enzym 54(3-4):72-75

Chen JD, Yi RZ, Lin YM, Feng DQ, Zhou HC, Wang ZC (2011) Characterization of terpenoids from the root of Ceriops tagal with antifouling activity. Int J Mol Sci 12(10):6517-6528

Chen G, Liu J, Jiang L, Ran X, He D, Li Y, Huang B, Wang W, Liu D, Fu S (2018) Peiminine protects dopaminergic neurons from inflammationinduced cell death by inhibiting the ERK1/2 and NF-KB signalling pathways. Int J Mol Sci 19(3):821

Chi YF, Kao YS, Chang KJ (1936) The alkaloids of fritillaria roylei I. Isolation of peimine1. J Am Chem Soc 58(7):1306-1307

Chopra RN, Nayar SL, Chopra IC (1956) Glossary of Indian medicinal plants. Council of Scientific \& Industrial Research, New Delhi, Vol. 1, pp. 138-139

Choudhary MI, Farooq A, Anjum S, Baumgold J, Sener B (1998) Structureactivity relationships of imperialine derivatives and their anticholinergic activity. Planta Med 64(02):172-174

Cho IH, Lee MJ, Kim JH, Han NY, Shin KW, Sohn Y, Jung HS (2011) Fritillaria ussuriensis extract inhibits the production of inflammatory cytokine and MAPKs in mast cells. Biosci Biotechnol Biochem 75(8):1440-1445

Da-Cheng HAO, Xiao-Jie G, Pei-Gen XIAO, Yong PENG (2013) Phytochemical and biological research of Fritillaria medicine resources. Chin J Natl Med 11(4):330-344

Dang D, Liu XF (2013) Study on study on the determination of total flavonoid content in Bulbus Fritillaria pallidiflorae and its anti-oxidative activity. Farm Mach 3:79-81

Day PD, Berger M, Hill L, Fay MF, Leitch AR, Leitch IJ, Kelly LJ (2014) Evolutionary relationships in the medicinally important genus Fritillaria $\mathrm{L}$ (Liliaceae). Mol Phylogenet Evol 80:11-19

Dong M, Wu LJ, Chen Q, Wang BX (2001) Isolation and identification of steroidal saponins from Dioscorea panthaica Prain et Burkill. Acta Pharm Sin 36:42-45

Du SF (1996) Comparison of pharmacological effects between Fritillaria Cirrhosa D. Don and Fritillariaeussuriensis Maxim. Tradit Chin Drug Res Clin 7:45-46

Duan B, Huang L, Chen S (2012) Chemical fingerprint analysis of Fritillaria delavayi Franch by high-performance liquid chromatography. J Sep Sci 35(4):513-518
Du W, Zhang H, Yue X, Zhu T, Ge W (2018) The analysis on volatile components of Zhejiang Fritillary sliceswith different primary processing methods. Lishizhen Med Mater Med Res 29:73-76

Eglen RM, Harris GC, Cox H, Sullivan AO, Stefanich E, Whiting RL (1992) Characterization of the interaction of the cervane alkaloid, imperialine, at muscarinic receptors in vitro. NaunynSchmiedeberg's Arch Pharmacol 346(2):144-151

Ellis PD, Dunlap RB, Pollard AL, Seidman K, Cardin AD (1973) Carbon-13 nuclear magnetic resonance of 5-substituted uracils. J Am Chem Soc 95(13):4398-4403

Erika L, Rebecca JF (2005a) Gale encyclopedia of public health. Gale Cengage Learning.

Erika L, Rebecca JF (2005b) Screening of antimicrobial and cytotoxic activities of Panamanianplants. Phytomedicine 1:149-153

Farooq A, Choudhary MI, Gilani AH, Shaheen F, Ali RA, Sener B (1994) A new anticholinergic steroidal alkaloid from Fritillaria imperialis of Turkish origin. Planta Med 60(04):377-379

Fiorentino A, D'Abrosca B, Pacifico S, Mastellone C, Piscopo V, Caputo $R$ (2008) Isolation and structure elucidation of antioxidant polyphenols from quince (Cydonia vulgaris) peels. J Agric Food Chem $56: 2660-2667$

Furuya S, Takayama F, Mimaki Y, Sashida Y, Satoh K, Sakagami H (2001) Cytotoxic activity of saponins from Camassia leichtlinii against human oral tumor cell lines. Anticancer Res 21(2A):959-964

Geng Y, Tan NH, Jun Z, Kong LY (2004) Isolation and identification of steroid saponins from the fresh rhizomes of Dioscorea panthaica. Chin J Nat Med 2(1):25

Goebel W, Schrempf H (1972) Isolation of minicircular deoxyribonucleic acids from wild strains of Escherichia coli and their relationship to other bacterial plasmids. J Bacteriol 111(3):696-704

Guo CG, Liu XF, Wang YG, Dang D (2013) Study on extraction of the total alkaloids from Bulbus Fritillaria pallidiflora and its pharmacological activities. Food Eng 17:83-86

Hao DC, Gu XJ, Xiao PG, Peng Y (2013) Phytochemical and biological research of Fritillaria medicinal resources. Chin J Nat Med 11:330-344

Hayes PY, Lambert LK, Lehmann R, Penman K, Kitching W, De Voss JJ (2007) Complete $1 \mathrm{H}$ and $13 \mathrm{C}$ assignments of the four major saponins from Dioscorea villosa (wild yam). Magn Reson Chem 45(11):1001-1005

Helsper JPFG, Bücking M, Muresan S, Blaas J, Wietsma WA (2006) Identification of the volatile component (s) causing the characteristic foxy odor in various cultivars of Fritillaria imperialis L (Liliaceae). J Agric Food Chem 54(14):5087-5091

Hou SJ, Zou CC, Zhou L, Lei PS, Yu DQ (2006) Synthesis of three natural diosgenyl glycosides. J Asian Nat Prod Res 8(8):689-696

Huang EX, Li CS, Xu DM (1990) Studies on the alkaloid constituents of Fritillaria pallidiflora Schrenk. China J Chin Materia Med 15:39-41

Huang L, Duan B, Wang L, Wei D, Lu Q, Chen S (2011) Simultaneous determination of seven nucleosides and nucleobases in aqueous extracts of Fritillaria taipaiensis by HPLC-DAD. China J Chin Materia Med 36(5):585-588

$\mathrm{Hu}$ CH, Shang EN, Lin WH, Cai MS (1993) Studies on the chemical constituents of Fritillaria taipaiensis L. Acta Pharm Sin 28(7):516-521

Hu Z, Zong JF, Yili A, Yu MH, Aisa HA, Hou AJ (2018) Isosteroidal alkaloids from the bulbs of Fritillaria tortifolia. Fitoterapia 131:112-118

Ito S, Kato M, Shibata K, Nozoe T (1963) On the Alkaloid of Fritillaria verticillate WILD. Var. Thunbergii BAKER. II. The structure of verticine. Chem Pharmaceut Bull 11(10):1337-1340

Ito S, Fukasawa Y, Miyashita M (1976) Structure of imperialine. Tetrahedron Lett 36:3161

Jiang Y, Li H, Li P, Cai Z, Ye W (2005) Steroidal Alkaloids from the Bulbs of Fritillaria p uqiensis. J Nat Prod 68(2):264-267

Jiang Y, Li P, Li HJ, Yu H (2006) New steroidal alkaloids from the bulbs of Fritillaria puqiensis. Steroids 71(9):843-848

Jin XQ, Xu DM, Xu YJ, Cui DB, Xiao YW, Tian ZY, Lu Y, Zheng QT (1993) The structure identification of zhepiresionol. Acta Pharmaceut Sinica 28(3):212-215

Ji H, Li P, Yao L, Shu Z (1993) Studies on antitussive action of puqietinone. J China Pharmaceut Univ 24:95-95

Jung JH, Lee CO, Kim YC, Kang SS (1996) New bioactive cerebrosides from Arisaema amurense. J Nat Prod 59(3):319-322 
Kaneko KO, Naruse N, Haruki K, Mitsuhashi H (1980) Isobaimonidine, a new Fritillaria alkaloid from the aerial part of Fritillaria verticillata. Chem Pharm Bull 28(4):1345-1346

Kaneko K, Nakaoka U, Tanaka M, Yoshida N, Mitsuhashi H (1981a) Two steroidal alkaloids, hapepunine and anrakorinine, from the mature Fritillaria camtschatcensis. Phytochemistry 20(1):157-160

Kaneko K, Tanaka M, Nakaoka U, Tanaka Y, Yoshida N, Mitsuhashi H (1981 b) Camtschatcanidine, an alkaloid from Fritillaria camtschatcensis. Phytochemistry 20(2):327-329

Kaneko K, Katsuhara T, Mitsuhashi H, Chen Y, Hsu H, Shiro M (1985) Isolation and structure elucidation of new alkaloids from Fritillaria delavayi Franch. Chem Pharm Bull 33(6):2614-2617

Kaneko K, Katsuhara T, Kitamura Y, Nishizawa M, Chen YP, Hsu HY (1988) New steroidal alkaloids from the chinese herb drug, "Bei-mu." Chem Pharm Bull 36(12):4700-4705

Kang DG, Oh H, Cho DK, Kwon EK, Han JH, Lee HS (2002) Effects of bulb of Fritillaria ussuriensis Maxim. on angiotensin converting enzyme and vascular release of NO/cGMP in rats. J Ethnopharmacol 81:49-55

Khaniki GB (2003) Fruit and seed morphology in Iranian species of Fritillariasubgenus Fritillaria (Liliaceae). Pak J Bot 35:313-322

Kimura Y, Mizuno T, Kawano T, Okada K, Shimada A (2000) Peniamidienone and penidilamine, plant growth regulators produced by the fungus Penicillium sp. No. 13. Phytochemistry 53:829-831

Kim SH, Shin DS, Oh MN, Chung SC, Lee JS, Chang IM, Oh KB (2003) Inhibition of sortase, a bacterial surface protein anchoring transpeptidase, by $\beta$-sitosterol-3-O-glucopyranoside from Fritillaria verticillata. Biosci Biotechnol Biochem 67(11):2477-2479

Kim EJ, Yoon YP, Woo KW, Kim JH, Min SY, Lee HJ, Lee SK, Hong JH, Lee KR, Lee CJ (2016) Verticine, ebeiedine and suchengbeisine isolated from the bulbs of Fritillaria thunbergii Miq. inhibited thegene expression and production of MUC5AC mucin from human airway epithelial cells. Phytomedicine 23:95-104

Kitajima J, Komori T, Kawasaki T, Schulten HR (1982a) Basic steroid saponins from aerial parts of Fritillaria thunbergii. Phytochemistry 21(1):187-192

Kitajima J, Komori T, Kawasaki T (1982b) Studies on the constituents of the crude drug "Fritillariae bulbus". Il. On the diterpenoid constituents of fresh bulbs of Fritillaria thunbergii Miq. Chem Pharm Bull 30:3912-3921

Kitajima J, Noda N, Ida Y, Komori T, Kawasaki T (1982c) Studies on the constituents of the crude drug "Fritillariae bulbus". IV. On the diterpenoid constituents of the crudr "Fritillariae bulbus." Chem Pharm Bull 30:3922-3931

Kitamura Y, Nishizawa M, Kaneko K, Ikura M, Hikichi K, Shiro M, Chen YP, Hsu HY (1988) Ussurienine, a novel 5a-cevanine alkaloid from Fritillariaussuriensis maxim. Tetrahedron Lett 29(16):1959-1962

Kitamura Y, Nishizawa M, Kaneko K (1989a) Novel steroidal alkaloids from Fritillaria ussuriensis. Tennen Yuki Kagobutsu Toronkai Koen Yoshishu 31(4):562-569

Kitamura Y, Nishizawa M, Kaneko K, Ikura M, Hikichi K, Shiro M (1989b) New steroidal alkaloids having a novel seven ring skeleton from Fritillariaussuriensis Maxim. Tetrahedron 45(18):5755-5766

Kitazawa T, Hashiba K, Cao J, Unno T, Komori SI, Yamada M, Wess J, Taneike T (2007) Functional roles of muscarinic M2 and M3 receptors in mouse stomach motility: studies with muscarinic receptor knockout mice. Eur J Pharmacol 554(2-3):212-222

Koketsu M, Kim M, Yamamoto T (1996) Antifungal activity against food-borne fungi of Aspidistra elatior Blume. J Agric Food Chem 44(1):301-303

Konchar K, Li XL, Yang YP, Emshwiller E (2011) Phytochemical variation in Fritillaria cirrhosa D Don (Chuan Bei Mu) in relation to plant reproductive stage and timing of harvest. Econ Botany 65(3):283

Lee P, Kitamura Y, Kaneko K, Shiro M, Xu GJ, Chen YP, Hsu HY (1988) The structural elucidation of Fritillaria alkaloids from Fritillaria ebeiensis var. purpurea I. The structures of ebeienine, ebeiedine and ebeiedinone. Chem Pharmaceut Bull 36(11):4316-4329

Lee B, Kim EY, Kim JH, Min JH, Jeong DW, Jun JY, Cho CY, Sohn Y, Jung HS (2015) Antiallergic effects of peiminine through the regulation of inflammatory mediators in HMC-1 cells. Immunopharmacol Immunotoxicol 37:351-358

Liang XT (2004) The basic research of traditional chinese medicine. Science Press, Beijing
Liang J, Cao X, Li J (2011) Analysis of volatile components of flowers of Fritillaria thunbergii by GC-TOF-MS [J]. China J Chin Mater Med 36(19):2689-2692

Lin G, Ho YP, Li P, Li XG (1995) Puqiedinone, a novel 5a-cevanine alkaloid from the bulbs of Fritillaria puqiensis, an antitussive traditional chinese medicine. J Nat Prod 58(11):1662-1667

Lin BQ, Ji H, Li P, Jiang Y, Fang W (2006a) Selective antagonism activity of alkaloids from bulbs Fritillariae at muscarinic receptors: Functional studies. Eur J Pharmacol 551(1-3):125-130

Lin BQ, Ji H, Li P, Fang W, Jiang Y (2006b) Inhibitors of acetylchoine esterase in vitro- Screening of steroidal alkaloids from Fritillaria species. PlantaMedica 72:814-818

Lin M, Zhou Z, Wang L (2010) Protection effect of Fritillaria thunbergii aginst hyperthyroidism in rats and mice. China Pharm 21:1362-1363

Liu QH, Jia XG, Ren YF, Muhatal Liang XT (1984) Studies on the chemical constituents of Fritillaria walujewi L Acta Pharm Sin 19:894-898

Liu HN, Li F, Luo YM, Zhu WF, Yan DM, Huang XF (2007) Diterpenoids from bulbus of Fritillaria monanth. Yao xue xue bao= Acta pharmaceutica Sinica, 42(11):1152-1154

Liu J, Wang X, Xiang Z, Li Y (2008) Study on the principal component analysis method to determination oftrace elements of Fritillaria thunbergii Miq. Guangdong Trace Elem Sci 15:31-35

Liu J, Peng C, He CJ, Liu JL, He YC, Guo L, Zhou QM, Yang H, Xiong L (2014) New amino butenolides from the bulbs of Fritillaria unibracteata. Fitoterapia 98:53-58

Liu W, Zou F, Li D (2015) Studies on P-glycoprotein inhibitor of multidrug tumor in Bulbus Fritillariae Thunbergii. Chin J Surg Integr Tradit West Med 21:379-382

Li QH, Wu ZH (1986) Isolation and identification of alkaloids from Fritillaria anhuiensis SC Chen et SF Yin. Acta Pharmaceutica Sinica, 10

Li QH, Wu ZH, Zhang LL, Shao L (1988) Isolation and identification of alkaloids from Fritillaria ningguoensis SC Chen et SF Yin. Acta Pharm Sin 23:415-421

Li P, Li XG, Xu GJ, Kaneko K (1990a) Study on the chemical constituents of Fritillaria Puqiensis. J China Pharm Univ 21(4):198-200

Li P, Xu GJ, Xu LS, Jin RL, Yu GD, Liu MX (1990b) Studies on the Chinese drugs Beimu XII Surveys of original plants and macroscopic identification. J China Pharm Univ 21(1):19-25

Li P, Yukie K, Koh K, Motoo S, Xu GJ, Chen YP, Hsu HY (1992) Asteroidal alkaloid from Fritillaria ebeiensis. Phytochemistry 31:2190-2191

Li P, Ji H, Xu GJ, Xu LSJ (1993) Studies on the antitussive and expectorant effects of Chinese drug Beimu. J Chin Pharm Univ 24:360-362

Li P, Liu LN, Tan Y, Xu GJ (1994) Studies on the constituents of Fritillaria ebeiensis. J China Pharm Univ 25:7

Li P, Wang YX, Xu GJ, Xu LS (1995a) Antitumor activity of puqietinone, a novel alkaloid from the bulbs of Fritillaria puqiensis. J Chin Pharm Sci 4:217-219

Li P, Xu GJ, Xu LS (1995b) Active constituents of the bulbs of Fritillaria ebeiensis and their antitumor activity in mice. Phytother Res 9:460-462

Li SL, Chan SW, Li P, Lin G, Zhou GH, Ren YJ, Chiu FCK (1999) Pre-column derivatization and gas chromatographic determination of alkaloids in bulbs of Fritillaria. J Chromatogr A 859(2):183-19

Li SL, Li P, Lin G, Chan SW, Ho YP (2000) Simultaneous determination of seven major isosteroidal alkaloids in bulbs of Fritillaria by gas chromatography. J Chromatogr A 873(2):221-228

Li P, Zeng LJ, Li SL, Lin G (2002) The extraction of imperialine and imperialine$3 \beta$-glucoside from Fritillaria pallidiflora Schrenk and quantitative determination by HPLC-evaporative light scattering detection. Phytochemical Analysis: an International Journal of Plant Chemical and Biochemical Techniques 13(3):158-161

Li HJ, Jiang Y, Li P, Ye WC (2006) Puqienine F, a novel veratramine alkaloid from the bulbs of Fritillaria puqiensis. Chem Pharmaceut Bull 54(5):722-724

Li HJ, Jiang Y, Li P (2006b) Chemistry, bioactivity and geographical diversity of steroidal alkaloids from the Liliaceae family. Nat Prod Rep 23:735-752

Li HJ, Jiang Y, Li P (2009) Characterizing distribution of steroidal alkaloids in Fritillaria spp and related compound formulas by liquid chromatography-mass spectrometry combined with hierarchial cluster analysis. J Chromatogr A 1216(11):2142-2149

Li H, Huang W, Wen YQ, Gong GH, Zhao QB, Yu G (2010) Anti-thrombotic activity and chemical characterization of steroidal saponins from Dioscoreazingiberensis CH Wright. Fitoterapia 81 (8):1147-56 
Li Z, An C, Hu K, Zhou K, Duan H, Tang M (2013) Multidrug resistance reversal activity of total alkaloid from Fritillaria thunbergii on cisplatin-resistant human lung adenocarcinoma A549/DDP cells. Chin J Pharmacol Toxicol 27:315-320

Li Y, Yili A, Li J, Muhamat A, Aisa HA (2016) New isosteroidal alkaloids with tracheal relaxant effect from the bulbs of Fritillaria pallidiflora Schrenk. Bioorg Med Chem Lett 26:1983-1987

Lou Y, Cai H, Liu X, Tu S, Pei K, Zhao Y, Cao G, Li S, Qin K, Cai B (2014) Element analysis andcharacteristic identification of non-fumigated and sulfurfumigated Fritillaria thunbergii Miq. usingmicrowave digestion-inductively coupled plasma atomic emission spectrometry combined with Fouriertransform infrared spectrometry. Pharmacogn Mag 10:S30-S36

Lu HF, Sue CC, Yu CS, Chen SC, Chen GW, Chung JG (2004) Diallyl disulfide (DADS) induced apoptosis undergo caspase-3 activity in human bladder cancer T24 cells. Food Chem Toxicol 42:1543-1552

Lyu Q, Tou F, Su H, Wu X, Chen X, Zheng Z (2015) The natural product peiminine represses colorectal carcinoma tumor growth by inducing autophagic cell death. Biochem Biophys Res Commun 462:38-45

Maharjan BL, Devkota HK, Baral B (2012) In-vitro antimicrobial activity and phytochemical screening of fritillaria delavayi. Nepal J Sci Technol 12:85-90

Meng ZY, Xu SX, Li W, Sha Y (1999) New saponins from Anemarrhena asphodeloides Bge. Zhongguo Yaowu Huaxue Zazhi 9(4):294-298

Mimaki Y, Sashida Y (1990a) Sterodial saponins and alkaloids from the bulbs of lilium brownii var colchesteri. Chem Pharmaceut Bull 38(11):3055-3059

Mimaki Y, Sashida Y (1990b) Studies on the chemical constituents of the bulbs of fritillaria camtschatcensis. Chem Pharm Bull 38(4):1090-1092

Minakawa M, Baek H, Yamada YM, Han JW, Uozumi Y (2013) Direct dehydrative esterification of alcohols and carboxylic acids with a macroporous polymeric acid catalyst. Org Lett 15(22):5798-5801

Mitsuhashi H, Nagai U, Endo T (1969) Studies on constituents of fritillaria camtschatcensis KER-GAWLER. Chem Pharm Bull 17(11):2370-2373

Miyase T (1989) Acidic and phenolic lignans from Juniperus Sabina. Phytochemistry 28:3483-3485

Morimoto H, Kimata S (1960) Studies on the components of Fritillaria Thunbergii Miq. I. isolation of peimineand its new glycoside. Chem Pharm Bull 8:302-307

Negi SS, Srivastava RK, Bisht NS (2007) Med Arom Plants. Indian Forester, Dehradun (India)

Oh H, Kang DG, Lee S, Lee Y, Lee HS (2003) Angiotensin converting enzyme (ACE) inhibitory alkaloids from Fritillaria ussuriensis. Planta Med 69:564-565

Ono M, Takamura C, Sugita F, Masuoka C, Yoshimitsu H, Ikeda T, Nohara T (2007) Two new steroid glycosides and a new sesquiterpenoid glycoside from the underground parts of Trillium kamtschaticum. Chem Pharm Bull 55(4):551-556

Orhan I, Kartal M, Abu-Asaker M, Senol FS, Yilmaz G, Sener B (2009) Free radical scavenging properties and phenolic characterization of some edible plants. Food Chem 114(1):276-281

Ori K, Mimaki Y, Sashida Y, Njkaido T, Ohmoto T, (1992a) Persicanidine A, a novel cerveratrum alkaloid from the bulbs of Fritillaria persica. Chem Lett, 163-166

Ori K, Mimaki Y, Sashida Y, Nikaido T, Ohmoto T (1992b) Cerveratrum alkaloids from bulbs of Fritillaria persica. Phytochemistry 31(10):3605-3607

Ori K, Mimaki Y, Sashida Y, Nikaido T, Ohmoto T (1992c) Steroidal alkaloids from the bulbs of Fritillaria persica. Phytochemistry 31(12):4337-4341

Pae HO, Oh H, Choi BM, Oh GS, Paik SG, Jeong S, Hwang KM, Yun YG, Chung HT (2002) Differentiation-inducing effects of verticinone, an isosteroidal alkaloid isolated from the bulbus of Fritillaria ussuriensis, on human promyelocytic leukemia HL-60 cells. Biol Pharm Bull 25(11):1409-1411

Pallen MJ, Lam AC, Antonio M, Dunbar K (2001) An embrassment of sortase -a richness of substrates? Trends Microbiol 9:97-101

Park JH, Lee B, Kim HK, Kim EY, Kim JH, Min JH, Kim S, Sohn Y, Jung HS (2017) Peimine inhibits the production of proinflammatory cytokines through regulation of the phosphorylation of NF-_B and MAPKs in HMC-1 Cells. Pharmacogn Mag 13:359-364

Peng W, Han T, Liu QC, Qin LP (2012) Chemical constituents of the flower of Fritillaria thunbergii. Chem Natl Compds, pp 1-2

Perry LM (1980) Medicinal plants of east and South East Asia. The MIT Press, Cambridge, pp.236-237

Ping L, Kitamura Y, Kaneko K, Shiro M, Xu GJ, Chen YP, Hsu HY (1992) A steroidal alkaloid from Fritillaria ebeiensis. Phytochemistry 31(6):2190-2191
Pi HF, Ruan HL, Zhan YH, Wu JZ (2006) Two new steroidal alkaloids from bulbs of Fritillaria lichuanensis. J Asian Natl Prod Res 8(3):253-257

Pi HF, Ruan HL, Zhang YH, Niu LM, Wu JZ (2006b) Steroidal alkaloids from bulbs of Fritillaria lichuanensis. J Asian Nat Prod Res 8:133-136

Pi HF, Zhang P, Zhu T, Ruan HL, Zhang YH, Sun HD, Wu JZ (2007) A new cycloartane triterpenoid from the leaves and stems of Fritillaria hupehensis

Pi HF, Zhang P, Ruan HL, Zhang YH, Sun HD, Wu JZ (2009) Two new triterpenoids from the leaves and stems of Fritillaria hupehensis. J Asian Natl Prod Res 11(9):779-782

Popova MP, Chinou IB, Marekov IN, Bankova VS (2009) Terpenes with antimicrobial activity from Cretan propolis. Phytochemistry 70(10):1262-1271

Qian ZZ, Nohara T (1995) Steroidal alkaloids of Fritillaria maximowiczii. Phytochemistry 40(3):979-981

Qian BC, Xu HJ (1985) Studies on the antitussive and sedative activities of peimine and peiminine. Yao Xue Xue Bao 20(4):306-308

Qu SY, Jiang XL, Chen YL, Fan G (1990) The pharmacological studies on the bulbs, leaves and stems of Fritillariaeussuriensis Maxim. Chin Spec Wild Econ Anim Plant Res 1:19-20

Rix E (1977) Fritillaria L. (Liliaceae) in Iran. Iran J Bot 1:75-95

Rix EM (2001) Fritillaria. A revised classification. The fritillaria group of the alpine garden society. UnitedKingdom

Ruan HL, Zhang YH, Wu JZ, Sun HD, Fujita T (2002) Two new diterpenoid dimers, fritillebinide $D$ and $E$, from bulbs of Fritillaria ebeiensis. J Asian Natl Prod Res 4(4):309-314

Ruan X, Yang L, Cui WX, Zhang MX, Li ZH, Liu B, Wang Q (2016) Optimization of supercritical fluid extraction of total alkaloids, peimisine, peimine and peiminine from the bulb of Fritillaria thunbergii Miq, and evaluation of antioxidant activities of the extracts. Materials 9(7):524

Sashida Y, Mimaki Y, Shimomura H (1989) Isolation and structure of kuroyurinidine, a new jerveratrum alkaloid from Fritillaria camtschatcensis, Chern Lett 897

Sener B (1994) Recent results in the search for bioactive compounds from Turkish medicinal plants. Pure Appl Chem 66(10-11):2295-2298

Shaheen H, Qureshi R, Akram A, Gulfraz M (2014) Inventory of medicinal flora from Thal desert, Punjab, Pakistan. Afr J Tradit Complement Altern Med $11: 282-290$

Shang ZJ, Liu XL (1995) The history of medicinal use of Beimu and investigation of its original plants. Chin J Med History 25(1):38-42

Shao B, Guo HZ, Cui YJ, Ye M, Han J, Guo DA (2007) Steroidal saponins from Smilax China and their anti-inflammatory activities. Phytochemistry 68(5):623-630

Shen S, Chen CJ, Bu R, Ga L, Li GY, Tan Y, Li X, Wang JH (2011) Three new steroidal saponins from Fritillaria pallidiflora. J Asian Natl Prod Res 13(11):1014-1022

Shen S, Li G, Huang J, Chen C, Ren B, Lu G, Tan Y, Zhang J, Li X, Wang J (2012a) Steroidal saponins from Fritillaria pallidiflora Schrenk. Fitoterapia 83(4):785-794

Shen S, Li G, Huang J, Tan Y, Chen C, Ren B, Lu G, Zhang C, Li X, Wang J (2012b) Chemical constituents from Fritillaria pallidiflora Schrenk. Biochem Syst Ecol 45:183-187

Shimomura H, Sashida Y, Mimaki Y, lida N (1988) Regaloside A and B, acylated glycerol glucosides from Lilium regale. Phytochemistry 27(2):451-454

Shou QY, Tan Q, Shen ZW (2009) A novel sulfur-containing diterpenoid from Fritillaria anhuiensis. Tetrahedron Lett 50(28):4185-4187

Shun-Wan CHAN, Ping LI, Yiu-Wa KWAN, Ge LIN (2011) In vitro tracheobronchial relaxation of Fritillaria alkaloids. Chin J Natl Med 9(5):345-353

Singh AP (2006) Ashtavarga: rare medicinal plants. Ethnobotanical Leaflets 1:11

Southon IW, Buckingham J (eds) (1989) Dictionary of alkaloids. CRC Press, Boca Raton

Stueber D, Grant DM (2002) $13 \mathrm{C}$ and 15N Chemical shift tensors in adenosine, guanosine dihydrate, $2^{\prime}$-deoxythymidine, and cytidine. J Am Chem Soc 124(35):10539-10551

Suh WS, Lee SY, Park JE, Kim DH, Kim S, Lee KR (2018) Two new steroidal alkaloids from the bulbs offritillaria thunbergii. Heterocycles 96:921-930

Thomson GE (2007) A report for the rural industries research and development corporation. The health benefits of traditional Chinese plant medicines: weighingthe scientific evidence. Australian Government

Tian J, Yin Y, Sun H, Luo X (2002) Magnesium chloride: an efficient 13C NMR relaxation agent for amino acids and some carboxylic acids. J Magn Reson 159(2):137-144 
Tong X (2016) Reversing multi-drug resistance on tumor cells and pharmacokinetics study on ingredients in a traditional Chinese medicine Fritillaria thunbergii Miq. Master's Thesis, Yunnan University of TraditionalChinese Medicine, Kunming, China

Tsukamoto Y (1989) The grand dictionary of horticulture. Shogakukan, Tokyo $4: 27$

Tsukamoto S, Kato H, Hirota H, Fusetani N (1995) Pipecolate derivatives, anthosamines $A$ and $B$, inducers of larval metamorphosis in ascidians, from a marine aponge Anthosigmella aft. raromicrosclera. Tetrahedron 51:6687-6694

Turktas M, Aslay M, Kaya E, Ertugrul F (2012) Molecular characterization of phylogenetic relationships in Fritillaria species inferred from chloroplast trnL-trnFsequences. Turk J Biol 36:552-560

Uprety Y, Asselin H, Boon EK, Yadav S, Shrestha KK (2010) Indigenous use and bio-efficacy of medicinal plants in the Rasuwa District, Central Nepal. J Ethnobiol Ethnomed 6(1):3

Utegenova LA, Nurlybekova AK, Duysebaeva MA, Jenis J (2019) Chemical constituents of the roots of Fritillaria pallidiflora. News Natl Acad Sci Republic of Kazakhstan Ser Chem Technol 1:32-38

Wang FP, Zhang R, Tang XY (1992) Revision of structure of peimisine. Acta Pharmaceut Sinica 27(4):273-278

Wang X, Yang K, Du L (2007) Determination of the proportion of Cu, Fe, Zn, and Ca elements in ThunberyFritillary Bulb and Tendrilleaf Fritillary Bulb. Sichuang Food Ferment 43:65-67

Wang D, Zhu J, Wang S, Wang X, Ou Y, Wei D, Li X (2011) Antitussive, expectorant and anti-inflammatory alkaloids from Bulbus FritillariaeCirrhosae. Fitoterapia 82(8):1290-1294

Wang D, Wang S, Chen X, Xu X, Zhu J, Nie L, Long X (2012) Antitussive, expectorant and anti-inflammatory activities of four alkaloids isolated from Bulbus of Fritillaria wabuensis. J Ethnopharmacol 139(1):189-193

Wang DD, Feng Y, Li Z, Zhang L, Wang S, Zhang CY (2014) In vitro and in vivo antitumor activity of Bulbus Fritillariae Cirrhosae and preliminary investigation of its mechanism. Nutr Cancer 66:1-12

Wang D, Jiang Y, Wu K, Wang S, Wang Y (2015) Evaluation of antitumor property of extracts and steroidal alkaloids from the cultivated Bulbus Fritillariaeussuriensis and preliminary investigation of its mechanism of action. BMC Complement Altern Med 15(1):29

Warrier PK, Nambiar VPK, Ramamurthy CR (1994) Indian medicinal plants. Orient Longman, Chennai (India)

Wei XH, Yang SJ, Liang N, Hu DY, Jin LH, Xue W (2013) Chemical constituents of Caesalpinia decapetala (Roth) Alston. Molecules 18:1325-1336

White JD, Badger RA, Kezar HS, Pallenberg AJ, Schiehser GA (1989) Structure, synthesis and absolute configuration of leptosphaerin, a metabolite of the marine ascomycete Leptosphaeria oraemaris. Tetrahedron 45:6631-6644

Wu Z, Zheng S (1997) Grey relational analysis and determination of elements in Fritillaria Thunbergii Bulb andFritillariaeCirrhosae Bulb. J Chin Med Mater 20:291-293

Wu JZ, Pan XP, Lou MA, Wang XS, Ling DK (1989) Studies on the chemical constituents of Fritillaria in Hubei X. Isolation and identification of alkaloids from Fritillaria ebeiensis var purpurea GD Yu et P. Li. Acta pharmaceutica Sinica 24(8):600-605

Wu JZ, Morizane C, lida A, Ueda SI, Zhou ZL, Xu M, Zhang M, Li RM, Fujita T (1995) Structures of three new diterpenoids, fritillebic acid and fritillebins A and B, from bulbs of Fritillaria ebeiensis GD Yu et GQ Ji. Chem Pharmaceut Bull 43(9):1448-1453

Wu JZ, Ruan HL, Zeng CL, Cheng HA, Zhang F, Zhao QS, Sun HD, Fujita T (1999) Structures of two new diterpenoid dimers from bulbs of Fritillaria ebeiensis. J Asian Nat Prod Res 1(4):251-257

Wu X, Chan SW, Ma J, Li P, Shaw PC, Lin G (2018) Investigation of association of chemical profiles with thetracheobronchial relaxant activity of Chinese medicinal herb Beimu derived from various Fritillaria species. J Ethnopharmacol 210:39-46

Xiao CP, Zhao HR, Li P, Xu GJ (1992) Antimicrobial activity (in vitro) of the constituents of bulbus Fritillariae. J China Pharm Univ 23:188-189

Xiao PG, Jiang Y, Li P, Luo YB, Liu Y (2007) The botanical origin and pharmacophylogenetic treatment of Chinese material medica Beimu. Acta Phytotaxon Sin 45:473-487

Xiao CM, Huang J, Zhong XM, Tan XY, Deng PC (2009) Two new homo-arocholestane glycosides and a new cholestane glycoside from the roots and rhizomes of paris polyphylla var pseudothibetica. Helvetica Chimica Acta 92(12):2587-2595

Xu FZ (2009) Studies on the antiasthmatic effect and mechnisms of the total alkaloids from fritillaria hupehensis in guinea pigs. LiShiZhen Med 20:1335-1337

Xu DM, Huang EX, Wang SQ, Wen XG, Wu XY (1990a) Studies on the alkaloids of Fritillaria pallidiflora. Acta Bot Sin 32:789-793

Xu DM, Arihara S, Shoji N, Yang XW, Huang EX, Li CS (1990b) Isolation and identification of yibeinoside A. Acta Pharmaceut Sinica 25(10):795-797

Xu DM, Xu ML, Wang SQ, Hung EX, Wen XG, Arihara S, Shoji N (1990c) Two new steroidal alkaloids from Fritillaria ussuriensis. J Natl Prod 53(3):549-552

Xu DM, He CH, Wang SQ, Huang EX, Xu ML, Wen XG (1990d) Structure of pingbeimine $C$. Acta Pharm $\operatorname{Sin} 25(2): 127-130$

Xu YJ, Xu DM, Luo G, Huang EX, Wu XY, Jin XQ, Cui DB, Liu SY (1992) Isolation and identification of yibeissine. Acta phar $\operatorname{Sin} 27: 121-124$

Xu YJ, Xu DM, Cui DB, Huang EX, Jin XQ, Liu SY (1993) Extraction and structure elucidation of yibeinoside B from Fritillaria pallidiflora. Acta Pharm Sin 28(3):192-196

Xu J, Guo P, Liu C, Sun Z, Gui L, Guo Y, Yamakuni T, Ohizumi Y (2011) Neuroprotective kaurane diterpenes from Fritillaria ebeiensis. Biosci Biotechnol Biochem 75(7):1386-1388

Xu J, Liu C, Guo P, Guo Y, Jin DQ, Song X, Sun Z, Gui L, Ma Y (2011 b) Neuroprotective labdane diterpenes from Fritillaria ebeiensis. Fitoterapia 82(5):772-776

Xu WL, Liu M, Chen DL, Wang JZ (2014) Chemical constituents from bulbs of Fritillaria pallidiflora Schrenk. Biochem Syst Ecol 57:198-202

Xu J, Zhao W, Pan L, Zhang A, Chen Q, Xu K, Lu H, Chen Y (2016) Peimine, a main active ingredient of Fritillaria, exhibits anti-inflammatory and pain suppression properties at the cellular level. Fitoterapia 111:1-6

Yan XY (2005) Effects of ethanol extract of three kinds of bulb fritillariaecirrhosae on guinea pigs with allergic asthma. Sichuan University, Chengdu

Yang ZD, Duan DZ (2012) A new alkaloid from Fritillaria ussuriensis Maxim. Fitoterapia 83(1):137-141

Yang Q, Nie S, Weng X, Li L, Huang L (2005) Experiment studies on anti-tumor effect in vivo and in vitro ofAconitum carmichaelii Debx. and Fritillaria thunbergii Miq. used singly or matched. Chin J Exp Tradit Med Formulae 11:25-28

Yang XD, Zhu J, Yang R, Liu JP, Li L, Zhang HB (2007) Phenolic constituents from the rhizomes of Gastrodia elata. Nat Prod Res 21(2):180-186

Yao YH, Xu GH, Zhang JD (2008) Determination of trace elements in traditional Chinese medicine from Changbai Mountain by ICP-MS [J]. SpectrosC Spectral Anal 28(5):1165-1167

Yin J, Kouda K, Tezuka Y, Tran QL, Miyahara T, Chen YJ, Kadota S (2003) Steroidal glycosides from the rhizomes of Dioscorea spongiosa. J Nat Prod 66(5):646-650

Yokosuka A, Mimaki Y (2008) Steroidal glycosides from the underground parts of Trillium erectum and their cytotoxic activity. Phytochemistry 69(15):2724-2730

Yoshikawa K, Takadera T, Adachi K, Nishijima M, Sano H (1997) Korormicin, a novel antibiotic specifically active against marine gram-negative bacteria, produced by a marine bacterium. J Antibiot 50:949-953

Yu GD, Li P, Xu GJ, Xu LS, Lu YQ, Ji GQ, Yang CP, Chen GY, Yang MX (1985) Studies on the Chinese drug bei-mu IV medicinal plant resources of Fritillaria from Hupei province. J China Pharm Univ 20:25-32

Zhang W (2008) Study on the determination of alkaloid and nucleosides in various species of fritillaria. Hunan Normal University, Changsha, China, Master's Thesis

Zhang J, Lao A, Ma G, Xu R (1991) Studies on chemical constituents of Fritillaria thunbergii Miq. II.Acta Pharm. Sin 33:923-926

Zhang J, Lao A, Huang H, Ma G, Xu R (1992) Studies on chemical constituents of Fritillaria thunbergii Miq. III. isolation and identification of Zhebeinone. Acta Pharm Sin 27:472-475

Zhang J, Lao A, Xu R (1993a) Studies on chemical constituents of Fritillaria thunbergii Miq. IV Chin Bull Bot 35:238-241

Zhang JX, Lao AN, Xu RS (1993b) Two new steroidal alkaloids, dongbeinine and dongbeirine, from Fritillaria thunbergii Miq. var. chekiangensis. Chin Chem Lett 4:321-321

Zhang J, Lao A, Xu R (1993c) Studies on the chemical constituents of fresh bulbs of Fritillaria thunbergii Miq. China J Chin Mater Med 18:354-355

Zhang JX, Lao AN, Xu RS (1993d) Studies on chemical constituents of Fritillaria yuminensis. Acta Bot $\operatorname{Sin}$ 35:963-967 
Zhang M, Shen Y, Zhu Z, Wang H, Ma D (1998a) Study on the anti-inflammatory and anti-diarrhea effects ofFritillaria thunbergii Miq. Hunan Guiding. J Tradit Chin Med Pharmacol 4:30-31

Zhang M, Shen Y, Zhu Z, Wang H, Li F (1998b) Study on the anti-ulcer and analgesia effects of Fritillariathunbergii Miq. Northwest Pharm J 13:208-209

Zhang YH, Ruan HL, Zeng FB, Pi HF, Zhao W, Wu JZ (2003) Effective part screening on antitussive, expectorant and antiasthmatic activities of Fritillariahupehensis. Zhongcaoyao 34:1016-1018

Zhang X, Geoffroy P, Miesch M, Julien-David D, Raul F, Aoudé-Werner D (2005a) Gram-scale chromatographic purification of $\beta$-sitosterol: synthesis and characterization of $\beta$-sitosterol oxides. Steroids 70:886-895

Zhang Y, Lu Y, Mao L, Proksch P, Lin WH, Tagalsins IJ (2005b) Two novel tetraterpenoids from the mangrove plant, Ceriops tagal. Org Lett 7:3037-3040

Zhang YH, Yang XL, Zhou XF, Ruan HL, Pi HF, Wu JZ, Sun HD (2007a) Alkaloids from Fritillaria hupehensis. Chin J Chem 25(11):1728-1731

Zhang YH, Yang XL, Zhou XF, Ruan HL, Pi HF, Wu JZ, Sun HD, Fujita T (2007) A new veratramine alkaloid from the bulbs of Fritillaria hupehensis. Chinese Chem Lett 18(2):175-177

Zhang YH, Yang XL, Zhang P, Zhou XF, Ruan HL, Pi HF, Wu JZ, Sun HD (2008a) Cytotoxic alkaloids from the bulbs of Fritillaria hupehensis. Chem Biodivers 5(2):259-266

Zhang Y, Zhang YJ, Jacob MR, Li XC, Yang CR (2008b) Steroidal saponins from the stem of Yucca elephantipes. Phytochemistry 69(1):264-270

Zhang J, Song C, Chen B (2010) Simultaneous determination of 5 nucleotides in Bulbus Fritillariae by RP-HPLC [J]. China J Chin Materia Med 35(1):67-70

Zhang QJ, Zheng ZF, Yu DQ (2011) Steroidal alkaloids from the bulbs of Fritillaria unibracteata. J Asian Natl Prod Res 13(12):1098-1103

Zhang HJ, Luo J, Shan SM, Wang XB, Luo JG, Yang MH, Kong LY (2013) Aphanamenes $A$ and $B$, two new acyclic diterpene [4 +2$]$-cycloaddition adducts from Aphanamixis grandifolia. Org Lett 15:5512-5515

Zhang C, Sun L, Chen R, Su JH, Zhang HF, Gu BR, Xing YW, Xue M (2016) Multiple analytical methodsfor identification and quality evaluation of
FritillariaeThunbergii Bulbus based on biological single moleculesby high-performance liquid chromatography. J Sep Sci 39:3536-3543

Zhang W, Xu T, Wang G (2018) Effect of extracts from Fritillaria thunbergii on oral ulcer model in rats. Mod Pract Med 30:242-243

Zheng Z, Xu L, Zhang S, Li W, Tou F, He Q, Rao J, Shen Q (2017) Peiminine inhibits colorectal cancer cell proliferation by inducing apoptosis and autophagy and modulating key metabolic pathways. Oncotarget 8:47619-47631

Zhou Y, Ji H, Li P, Jiang Y (2003) Antimuscarinic function of five Fritillaria alkaloids on guinea pig tracheal strips. J China Pharmaceut Univ 34(1):58-60

Zhou Y, Ji H, Lin BQ, Jiang Y, Li P (2006) The effects of five alkaloids from Bulbus Fritillariae on theconcentration of CAMP in HEK cells transfected with muscarinic M2 receptor plasmid. Am J Chin Med 34:901-910

Zhou JL, Jiang Y, Bi ZM (2008) Study on nucleosides from Fritillaria puqiensis. Chin Pharm J 43(12):894-896

Zhou JL, Xin GZ, Shi ZQ, Ren MT, Qi LW, Li HJ, Li P (2010) Characterizationand identification of steroidal alkaloids in Fritillaria species using liquidchromatography coupled with electrospray ionization quadrupoletime-offlight tandem mass spectrometry. J Chromatogr A 1217:7109-7122

Zhou Y, Gao X, Wu C, Wu Y (2014) Bioaccessibility and safety assessment of trace elements from decoctionof "Zhebawei" herbal medicines by in vitro digestion method. J Trace Elem Med Biol 28:173-178

Zhou M, Ma X, Ding G, Wang Z, Liu D, Tong Y, Zhou H, Gao J, Hou Y, Jiang $M$ (2017) Comparisonand evaluation of antimuscarinic and antiinflammatory effects of five Bulbus Fritillariae species based onUPLC-Q/ TOF integrated dual-luciferase reporter assay, PCA and ANN analysis. J Chromatogr B 1041-1042:60-69

\section{Publisher's Note}

Springer Nature remains neutral with regard to jurisdictional claims in published maps and institutional affiliations.

\section{Submit your manuscript to a SpringerOpen ${ }^{\circ}$ journal and benefit from:}

- Convenient online submission

- Rigorous peer review

- Open access: articles freely available online

- High visibility within the field

- Retaining the copyright to your article

Submit your next manuscript at $\boldsymbol{\nabla}$ springeropen.com 